\title{
Characteristics of Synchrotron Radiation and of its Sources
}

S. Krinsky, M. I. Perlman, and R. E. Watson

\section{Brookhaven National Laboratory}

\section{Upton, NY 11973}

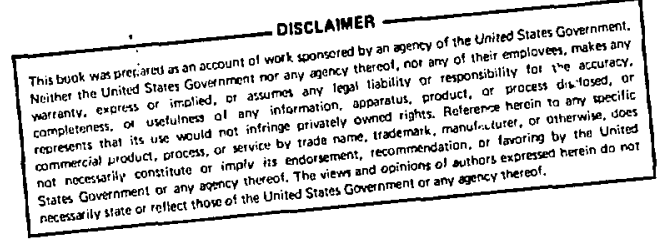




\section{ACRATONLEDGMENT}

Collaboration with Ren Green and Rena Chasman during their

Iffetimes was a real pleasure and has strongly influenced the content of this chapter. 
Section or Equations

Where Defined or Used

$(75-76)$

(113) and Sec. 3.11

(83), (84) $\begin{aligned} a_{x}(s), a_{z}(s) & \text { Twiss parameters, which, along with } \\ & \beta_{x}(s), \beta_{z}(s), Y_{x}(s), Y_{z}(s), \text { specify. }\end{aligned}$ the Courant-Snyder Invariant

B Magnetic fleld

B Amplitude of a wiggler or uniulator (254), (289)

B. Normalized electron velocity

$B_{x}(s), B_{z}(B)$ Amplitude function

(78). - (87)

$\beta^{*}, \beta_{h}^{*}$ Velocity of the moving frame (equal to the average longitudinal electron

(261), (293) velocity) in a wiggler or unculator

c Speed of 1ight

$d I(\omega) / d \Omega$ Energy radiated per unit frequency interval, per unit solid angle

(44), (51), (269)

e (s) Unit vector parallel to Ideal orbit

$\mathrm{e}_{\mathrm{x}}(\mathrm{s}), \mathrm{e}_{z}(\mathrm{~s})$ Unit vectors normal to Ideal orbit

E Electric field

E Electron energy

$E_{0}$ Energy of electron in ideal orbit

$\varepsilon$ Energy deviation. of electron from ideal energy $\mathrm{E}_{0}$

$\varepsilon_{\max }$ Maximum energy deviation accepted by the rf aystem

$\varepsilon_{x}, \varepsilon_{z}$ mittances, the Courant-Snyder invarients; also designate ' the damped equilibrium (82), $(84) ;(170),(178)$ enittances 
Principal Symbols (continued)

$\left.\stackrel{E}{ }\right|^{\prime} E_{1} \begin{aligned} & \text { Init vectors perpendicular to the } \\ & \text { direction of observation }\end{aligned}$

f Fraction of total photon flux which is parallel polarized

$F$ On axis $\left(0^{\circ}\right)$ photon flux density

I Total photon flux function

G Photon power distribution function

G(s) Inverse of orbit bending radius at points along orbit

Y Ratio of electron energy to its rest energy

$\gamma^{*} \gamma$ relevant to a frame for electrons passing through a transverse undulator

$\gamma_{h}^{*} \gamma$ relevant to a frame for electrons passing through a helical undulator

$Y_{I}$ Resonant electron energy in a free electron Iaser

h Accelerator harmonic number

$\mathrm{H}_{2}$ On axis photon density function

$n(8)$ Dispersion function

I Energy radiated per unit frequency

\& Electron current

$J_{x}, J_{z}, J_{\varepsilon}$ Damping partition numbers

k Harmonic number of characteristic radiation firom an undulator

$\mathrm{R}(\mathrm{s})$ Focusing function.

R Transwerse undulator scaling parameter

$K_{h}$ Helleal undulator scaling parameter

$K \equiv d t / d t '$ derivailve of the observer's time with respect to the retarded time
Section or Equations Where Defined or Used

FIg. 6

Eq. (12), $\mathrm{F} 18 \cdot 4$

Eq. (11), Fig. 2

(10), (224)

(61)

(1)

(264)

(295)

(312)

(104), (106)

(221), (222)

(43), (51), (54)

(271), (277)

(256a), (259) 
L Total length or "cIrcumference" of 3 reference orbit

$\mathcal{L}$ Length of a wiggler or undulator

$\lambda$ Photun wavelength

$\lambda_{c}$ Characteristic wavelength of synchroton,

$\lambda_{0}$ Length of a period of a wiggler or undulator

D Direction from electron to observer

iN Photon flux

$\eta$ Number of periods in a wiggler or undulator

$v_{z} v_{x}, v_{z} \begin{aligned} & \text { Tune, number of betatron oscillations } \\ & \text { per orbit revolutión }\end{aligned}$

w Photon frequency

$\omega_{c} \begin{aligned} & \text { Characteristic frequency of synchrotron } \\ & \text { radiation }\end{aligned}$

$\omega_{\text {rf }}$ rf frequency in the accelerating cavity

$w_{1}$ Fundamental radiation frequency from a trans-

$\omega_{1 \mathrm{~h}} \begin{aligned} & \text { Fundamental radiation frequency from a helical } \\ & \text { undulator }\end{aligned}$

$\Omega$ Solld angle

$\Omega_{h}$ Wiggle frequency (helical undulator)

$\Omega_{L}$ Oscillation frequency in a free electron laser

$\Omega_{s}$ Synchrotron obcillation frequency

$\Omega_{\mathrm{w}}$ Wiggle frequency (transverse undulator)

p Momentum

Po Momentum on the 1deal orbit

P Synchrotron radiation power

(7), (35) 
\$ Betatron oscillation phase

(78a)

(272)

\$o Azimuthal angle defining radiation direction from an undulator axis

$\phi_{s}$ Phase difference associated with synchrotron oscillation

Q Scalar potential

I Phase between EM waye and the electron motion

$\psi$ Photon angle with respect to electron velocity direction; in general this is an angle out of the orbit plane

R Distance between electron and observer

$p$ Bending radius of orbit

s Position along 1deal orbit

S Poynting's vector.

- A standard deviation for a number of quantities (as below)

$\sigma_{E}$ Standard deviation in electron energy from ideal orbit energy

$\sigma_{L}, \sigma_{\tau}$ One-half of electron bunch length in space and time respectively

$\sigma_{R}$ Synchrotron radiation cone half-angle $\sigma_{x}, \sigma_{z}, \sigma_{x}, \sigma_{z}, \begin{aligned} & \text { Standard deviations assoclated } \\ & \text { with the electron distribution }\end{aligned}$ about the 1deal orbit

$\sigma_{X}, \sigma_{Z}, \sigma_{Y},=\sqrt{\sigma_{Y^{\prime}}^{2}+\sigma_{R}^{2}}$ where $Y=X$ or $z_{;}$

Standard deviations associated with the angular properties of a photon source

To Perlod of an electron on the ideal orbit

I Time interval by which an electron precedes the synchronous electron in synchrotron oscillations

$\tau_{q}$ Quantum lifetime for betatron oscillations
(109) 
$\tau_{s}$ Quantum lifetime for synchrotron oscillations (202)

$\tau_{x}, \tau_{z}, \tau_{\varepsilon} \begin{aligned} & \text { Radial, vertical and energy damping } \\ & \text { times }\end{aligned}$

$u_{c}$ Characteristic energy of synchrotron

$u_{0}$ Energy lost per turn by an electron on the Ideal orbit

$(4),(36)$

$v$ Electron velocity

$\hat{\mathrm{v}}$ rf peak voltage

$\dot{x}$ Horizontal deviation from the Ideal orbit

Fig. 9

$\xi$ Normalized deviation of electron energy from resonant value in free electron laser

$y$ Used to represent $x$ or $z$ when an equation holds for toth horizontal and vertical betatron oscillations

2 Vertical deviation from the ideal orbit

Fig. 9

$\zeta$ Coordinate along the axis of a wiggler or undulator

\section{Derivatives:}

$\dot{f}$ Time derivative of function $f$

$f$ ' Spatial derivative of $f$ with respect to $s$, the coordinate along the ideal orbit 


\section{INTRODUCTION}

Synchrotron 11ght has a number of special characteristics which make electron synchrotron and storage ring sources powerful as research instruments and, potentially, as facilities for technological application as well. Synchrotron light has a continuous spectrum ranging from infrared to $x-r a y$ wavelengths, it is highly intense, and is strongly polarized. It is emitted in pulses of duration about $10^{-9}$ seconds, repeating usually at a rate of about $10^{6}$ per second; the emitting source area can be $\operatorname{small,~} 1 \mathrm{~mm}^{2}$ or even less; and the emission from any. point in the electron orbit is restricted to a narrow cone tangent to the curved orbit path. A single storage ring can provide a considerable number of ports at which equipment for utilizin? photon beams may be installed. It is the qualities of the radiation and the physics controlling them which are the subject matter of this chapter.

The properties of a synchrotron light beam depend both on the emission process Itself and on the electron optics of the storage ring or accelerator from which the light is obtained. Synchrotron light emission and the classical relativistic electromagnetic theory describing f.t are reviewed in Section 2 of this chapter; the electron optics of storage rings is considered In some detail in Section 3. Ne hope that this latter section will provide the reader not only with physical insight into the electron optics but also with the basic technical vocabulary of the accelerator scientist.

We begin with the geometry of the ideal electron orbit and develop the distribution which electrons take around it. In Section 4 this is folded with the properties of the synchrotron light emission process itself to define the effective photon source. Here we have relied heavily on a classic report of G. K. Green (1976). Detalled discussion of the relevance of particular source characteristics to specific classes of experiments will be 
found in the research chapters in this report. It is possible at this point, Section 5, to compare the predictions of classical relativistic theory with experiment, and as will be seen, one finds agreement witinin the experimental uncertainties, currently about one percent. These uncertainties arise from imprecision in such factors as the number, position, and energy of the electrons in the ring. Because the beam intensittes may thus be calculated from data about the electrons, there is the possibility of using a synchrotron light source as an absolute photon standard.

.. Through Sect. .5 of .this chapter, discussion will have concentrated un. the light emitted by electrons undergoing radial acceleration along orbit curvatures produced by the bending magnets of a storage ring or accelerator. In Sect. 6 we consider the possibility of inserting a "wiggler" or "undulator" magnet into one or more of the straight sections of a storage ring, producing a magnetic field alternating in polarity along the orbit and resulting in local deflections of the electrons, but no net deviation. The field strength of the wiggler magnet may be significantly higher than that of the bending magnets, yielding a source of harder photons. By having several periods or wiggles one obtains an orbit geometry different from that in a bending magnet, namely several radiation sources located on a line. This offers the possibility of flux enhancement; the optical characteristics of such a source are discussed. If there are many periods in the wiggler or undulator, the spectrum is changed. The electron undergoes a periodic perturbation and emits photons characteristic of this periodicity. Due to relativistic effects, the radiated wavelength is much shorter than the macroscopic scale of the field variations in the laboratory; it depends on the inverse square of the electron energy and is typically in the far ultraviolet. The form of the spectrum is different from that of normal synchrotron radiation and is 
characterized by peaks having central brightness several orders of magnitude greater than that available from bending magnets.

The posstbility of Inducing free electron laser action is also considered in Sect. 6. A free electron laser consists of an undulator magnet situated in an optical cavity. The combined action on the electron beam of the static undulator magnetic field and the radiation field in the cavity results in an amplification of the radiation present in the cavity. Not only is the output radiation of a free electron laser of great interest, but the output electron beam has the very important property that it is coherently modulated at the radiation wavelength. Whereas normal short wavelength synchrotron radiation is incoherent (intensity increases linearly with electron beam current), there may be coherent emission (intensity increases quadratically with electron current) if there are coherent density modulations of the electron beam on the scale of the radiated wavelength. 


\section{SYNCHROTRON RADIATION AND CLASSICAL ELECTROMAGNETIC THEOKY}

\subsection{Introduction}

When a charged particle is accelerated it emits electromagnetic radiation; however, very little energy is radiated unless the particle is highly relativistic. It is convenient to describe a relativistic particle in terms of a quantity $\gamma$, the ratio of its total energy to its rest energy. Thus if

$$
r \equiv \mathrm{E} / \mathrm{m}_{\mathrm{o}} \mathrm{c}^{2}
$$

the momentum is then

$$
p=r m_{0} v
$$

where $v$ is the velocity, $m_{0}$ is the particle's rest mass and $c$ the speed of 1ight. The acceleration is governed by the Lorentz equation

$$
\frac{d \underline{p}}{d t}=e\left(\underline{E}+\frac{\underline{v} \times \underline{B}}{c}\right) \text {, }
$$

where $\underline{E}$ and $\underline{B}$ are the electric and magnetic flelds and $e$ the particle

charge. (E is written as a vector quantity to differentiate it from the energy E.) Although it is not practicable to effect large acceleration of highly relativistic particles with electric fields, magnetic flelds of several kilogauss, normal to the direction of particle motion, produce transverse accelerations of relativistic electrons of such magnitude that copious radiation is emitted. The energy radiated per turn of the electron in such a magnetic field is 


$$
u_{0}=\frac{4 \pi e^{2} \gamma^{4}}{3 p} \text {, }
$$

where $\rho$ is the electron trajectory radius. The rest mass of a proton is n2000 times greater than that of an electron. Noting that at very high energies particle momenta become independent of rest mass, so that electrons and protons then have comparable $\rho$ values, it becomes clear that at the same kinetic energy the electron radiates $\sim(2000)^{4}$ or $\sim 10^{13}$ times as much as a proton. Electrons are thus used as the source of synchrotron radiation. For an electron

$$
\begin{aligned}
\gamma & =1957 \mathrm{E}(\mathrm{GeV}), \\
\rho \text { (meters) } & =\frac{33.35 \mathrm{E}(\mathrm{GeV})}{\mathrm{B}(\mathrm{k} \text { gauss) }}
\end{aligned}
$$

and Eq. (4) becomes

$$
U_{0}=88.5 \mathrm{E}^{4} / 0=2.65 \mathrm{E}^{3} \mathrm{~B},
$$

where $U_{0}$ is in $\mathrm{keV}$ per electron-turn. It follows that the power in kilowatts radiated in an electron storage ring at energy $E$ and current $A$ (in amps) is

$$
P=88.5 d^{4} E^{4} / \rho=2.65 \mathrm{E}^{3} \cdot \mathrm{U}_{\mathrm{B}}
$$

For describing the smooth continuous spectrum and the spatial

characteristics of the radiation, it is useful to define a characteristic wavelength or frequency 


$$
\begin{aligned}
& \lambda_{c} \equiv 4 \pi \rho / 3 \gamma^{3}=5.59 \rho / \mathrm{E}^{3}=186 / \mathrm{BE}^{2} \text { in } \AA \\
& \omega_{c} \equiv 2 \pi c / \lambda_{c}=3 c \gamma^{3} / 2 p .
\end{aligned}
$$

The radiated power as a function of wavelength for a $1 \%$ bandwidth and an azimuthal milliradian of the electron orbit is

$$
P=5.95 \times 10^{-8}\left(\gamma^{4} / / \rho\right) G_{2} \quad \text { ergs/sec. }
$$

Plotted in FIg. 1 is the wavelength dependent factor $G_{2}$, where

$$
\therefore \quad G_{n} \equiv\left(\lambda_{c} / \lambda\right)^{n} \int_{\lambda_{c} / \lambda}^{\infty} k_{5 / 3}(n) d n,
$$

where $k_{5 / 3}$ is a modified Bessel function. The peak of this power spectrum appears at $\sim_{2 / 3} \lambda_{c}$, and one-half of the total power is associated with wave lengths greater than $\lambda_{c}$, the other half with shorter wavelengths.

While power is of concern in connection with design of optical

elements used with synchrotron radiation of greater interest to the experimentalist is photon flux. This may be obtained by dividing the power by the photon energy inc/ $\lambda$, yielaing

$$
N=\frac{P \lambda}{h c}=1.256 \times 10^{11} \gamma \Omega G_{1} \equiv \gamma_{d} \ell \tau\left(\lambda / \lambda_{c}\right)
$$

where $\mathfrak{F}$ is plotted :n Fig. 2 and the flux $N$ is, as before, for a $1 \%$ bandwidth and for 1 mrad of the orbit arc. Photon flux drops off by two orders of magnitude from $\lambda_{c}$ to $0.16 \lambda_{c}$ but only by a factor of three from $\lambda_{c}$ to $1000 \lambda_{c}$. 


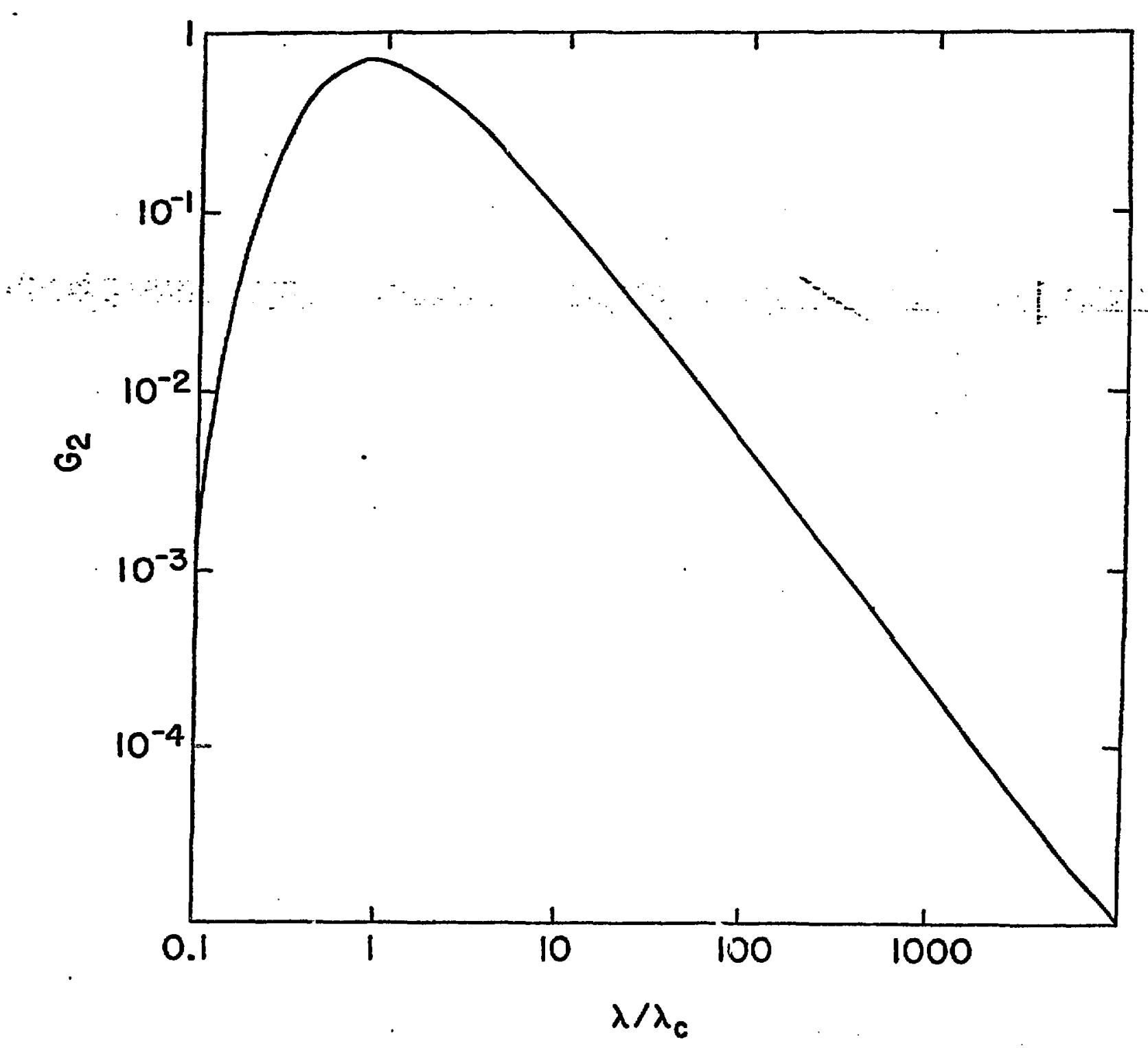

FIGURE 1 


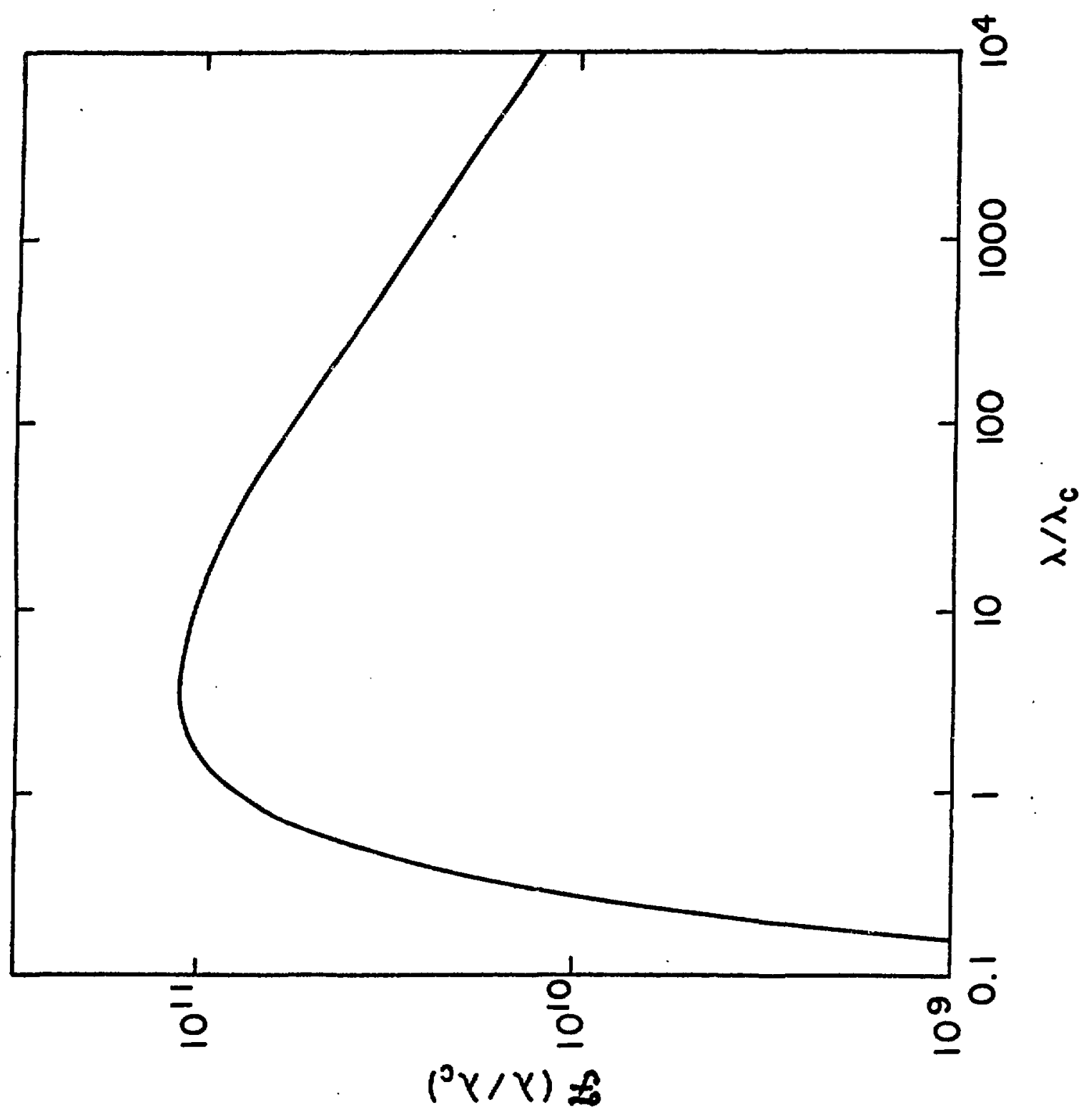


The radiation is highly directional; from any point in the electron orbit, it is concentrated in a narrow cone with apex angle of the order of a milliradian and axis tangent to the orbit. As the electron circulates it thus emits a sheet of radiation having a vertical spread of $u 1$ mrad. This radiation is strongly polarized. For emission in the orbit plane the electric vector lies in the plane and is, of course, normal to the emission direction. For emission at some angle $\psi$ with respect to the orbital plane, E has, as well, a component normal to the parallel compunent and to the direction of emission. The $\psi$ dependence of the photon flux associated with the parallel and perpendicular components of $\underline{E}$ is displayed In Fig. 3 for several values of $\lambda / \lambda_{c}$. The plots are normalized to the flux $\left[\frac{\partial N}{\partial \psi}\right]_{\psi=0} d \psi$ in the orbital plane for the wavelength in question, where for a current $\Omega_{\text {(amp) }}$

$$
\begin{aligned}
{\left[\frac{\partial N}{\partial \psi}\right]_{\psi=0} \mathrm{~d} \psi=} & 3.461 \times 10^{7} \ell \gamma^{2}\left(\frac{\lambda_{c}}{\lambda}\right)^{2} k_{2 / 3}^{2}\left(\lambda_{c} / 2 \lambda\right) \\
\equiv & \gamma^{2} d F\left(\lambda / \lambda_{c} ; \psi=0\right) \text { photons per } \\
& (17 \text { bandwidth, sec, } \\
& \text { millirad of orbit arc, } \\
& \text { mililirad of vertical angle } \psi) .
\end{aligned}
$$

The function F is plotted in Fig. 4. Note that the abscissa of Fig. 3 is $r$ tIIIss the angle $\psi$; in other words, the natural angular unit, for emission by an electron of energy $Y$, is $1 / \gamma$. For a $1-\mathrm{GeV}$ electron $\gamma$ is 22000 and the angular 


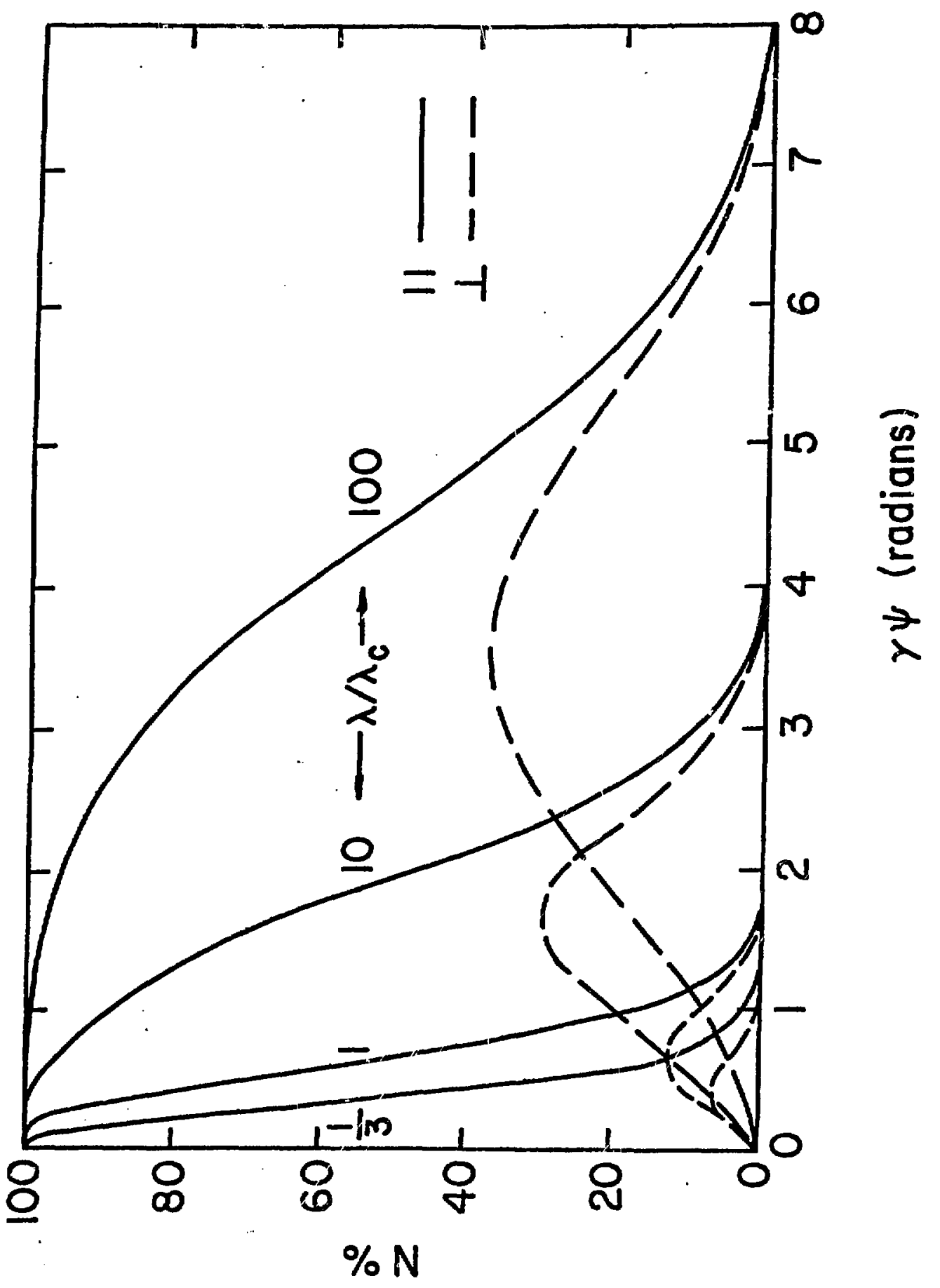


spread of photons of wavelength $\lambda_{c}$ is about half a milliradian, a very small angle. The spread increases with increasing wavelength and, in fact, for $\lambda / \lambda_{c} \gg 1$, the maxima in intensity are out of the orbital plane. An increase in spread implies a smaller fraction of the total Intensity at the center; as a result, the flux per unit angle for $\psi=0$, plotted in Fig. 4, falls off more rapidiy with increasing wavelergth than does the total flux plot of Fig. 2 .

A convenient diagram is provided In Fig. 5, where the fraction of parallel polarized radiation, integrated over $\psi$, is indicated as a function of $\lambda / \lambda_{c}$. In sumary then, if one utilizes a small vertical angle centered in the orbit plane, the radiation is effectively one-hundred percent parallel polarized, as Fig. 3 exhibits. If, however, a substantial vertical angle is utilized, the polarization, though substantial, is not entirely parallel, as Fig. 5 shows. Summed over wavelengths and $\psi$, the emission energy associated with the parallel component is seven times that of the perpendicular. 


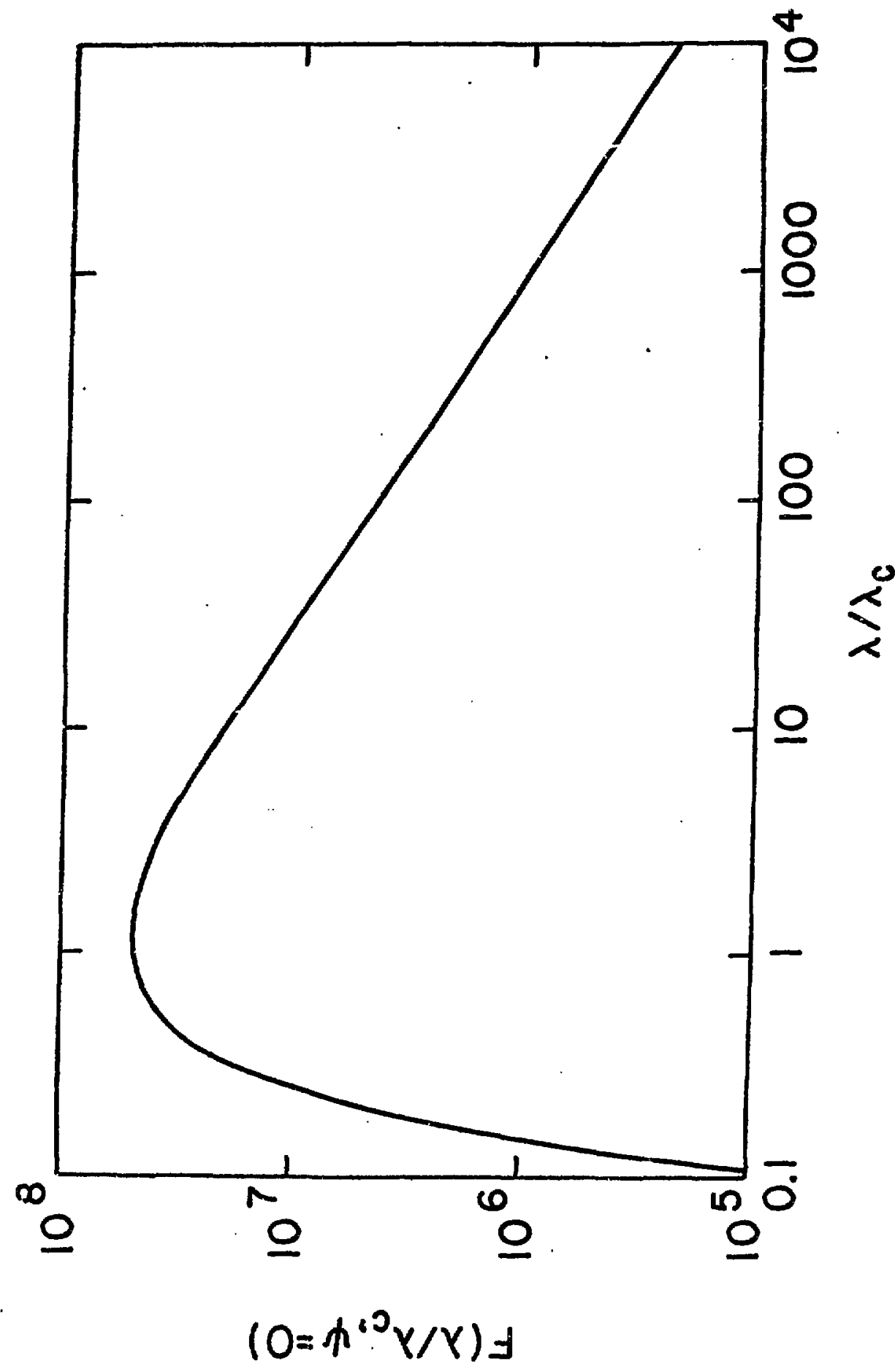




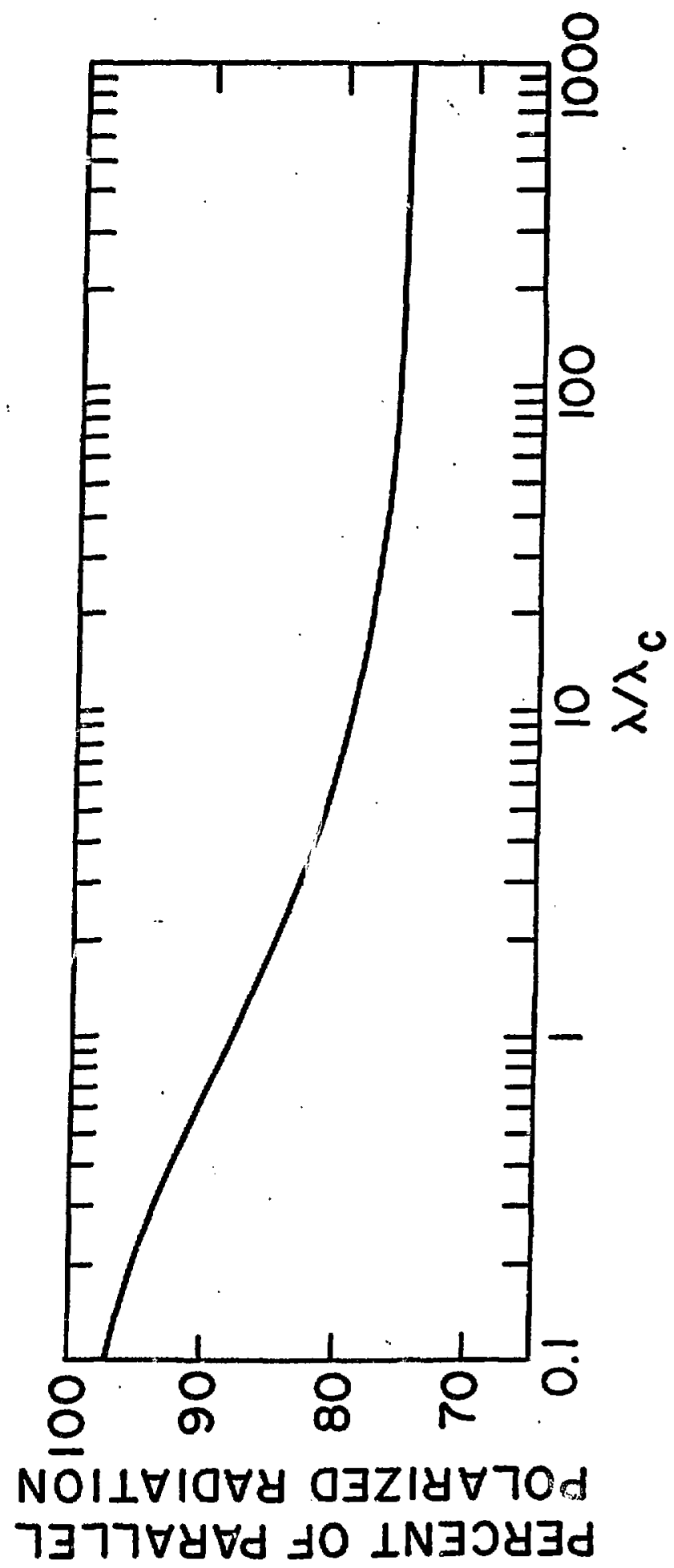


A radiofrequency cavity, incorporated as part of the orbit chamber,

makes up the energy losses due to the synchrotron radiation. Only those electrons

so bunched that they traverse the cavity correctly phased with respect to

the cavity roltage will remain at essentially constant energy and

In stable orbit. A bunch length of tens of centimeters raque-ing of the

order of a nanosecond to pass a polnt is typical; the repeat time between

sightings of the bunch, very roughly 1000 times longer, is just the

circumference of the ring divided by the electron speed. Because the

cavity frequency is usually much higher than the circulation frequency,

1t is possible to have more than one bunch in the ring at a time. High

electzon density in a bunch has deleterious effects on bunch spatial

stabllity; hence, to avoid undue shortening of beam lifetime at high

currents, large photon fluxes are achleved by having a number of bunches,

six, elght, or many more, in the ring.

In an actual accelerator the longitudinally bunched electrons are

found in a distribution of transverse positions and orbit directions about

the equilibrlum oroit. This has a variety of causes, the principal one being quantum Iuctuations associated with the discrete nature of photon 
emission. It turns out that the vertical dimension of the electron beam can be made much smaller than the horizontal dimension. Thus, the radiation source is a thin ribbon of electrons lying in the orbit plane. The consequences of having this source configuration will be aiscussed In Sect. 4 .

First, however, we will derive the equations for synchrotron radiation from a single electron, some of which have been considered above.

\subsection{Classical Theory}

Around the turn of the century Lienard (1898) derived the relation for the emission from a centrf.petally accelerated charge. classical relativiatic theory was applied to the problem by Schott. (1912) who, in fact, obtained most of what we require. It was not until the $1940^{\prime}$ s that serious concern over radiative loss from electrons in accelerators (Iwanenko anà Pomeranchuk 1944, McMillan 1945, Blewett 1946, Schiff 1946, and Schwinger 1946) led to detailed investigation of the effects. Schwinger (1949) and Sokolov and Ternov (1968) have worked out the relevant equations. This review draws heavily on the elegant presentation of Jackson (1975).

At a given observation point and time $t$ the scalar and vector potentials due to a point charge are the Lienard-Wiechert potentials

$$
\Phi(\underline{x}, t)=e[1 / k R]_{\text {ret }}
$$

and

$$
\underline{A}(\underline{x}, t)=e[\underline{B} / k R]_{r e t}
$$

where the brackets with subscript ret indicate that.the quantizy in brackets is to be evaluated at the retarded time $t^{\prime}=t-\left[R\left(t^{\prime}\right) / c\right]$; $B$ is the normalized electron velocity 


$$
\underset{\sim}{v} \equiv c \underset{\sim}{\beta}=c \sqrt{1-(1 / \gamma)^{2}}
$$

and

$$
\frac{d t}{d t^{\prime}} \equiv K=1+\frac{1}{c} \frac{d R}{d t^{\prime}}=1-\underline{n} \cdot \underline{\beta} .
$$

In Eq. (15), is 1s a unit vector directed from the position of the charge to the observation point and $R$ is the distance between points. If $\mathfrak{n}$ and $\underline{B}$, are parallel, the charge is viewed from a point towards which the charge is approaching; then $k \sim \gamma^{-2}$. If one introduces a four component $\beta_{\mu}=(\beta, 1)$, Eqs. (13) can be written

$$
A_{\mu}(x, t)=e \int \frac{\beta_{\mu}\left(t^{\prime}\right)}{R\left(t^{\prime}\right)} \delta\left[t^{\prime}+\frac{R\left(t^{\prime}\right)}{c}-t\right] d t^{\prime},
$$

where the $\mu=4$ component is $1 \phi$. Now $\underset{\mu}{\mathrm{E}}$ and $\underset{\mu}{\mathrm{B}}$ are related to these

potentials by

$$
\underline{B}=\nabla \times A
$$

and

$$
\underset{m}{E}=-\nabla \Phi-\frac{1}{i} \frac{\partial A}{\partial t}
$$

Because the only quantity which depends on spatial coordinates is the magnitude of $R$, the gradient operation becomes $\underset{\sim}{n} \frac{\partial}{\partial R}$ and

$$
\underset{\sim}{E}(x, t)=e \int\left[\frac{n}{R^{2}} \delta()+\frac{1}{C R} \underset{\sim}{(\beta-n)} \delta^{\prime}()\right] d t^{\prime}
$$




$$
\underset{M}{B}(x, t)=e \int\left(n \times \underset{\sim}{(n)}\left[-\frac{\delta()}{R^{2}}+\frac{1}{c R} \delta^{\prime}()\right] d t^{\prime}\right.
$$

the arguments of the $\delta$ functions are the same as in Eq. (16) and the primes indicate differentiation with respect to the arguments. If one integrates by parts on the derivative, $\delta^{\prime},(19)$ and (20) become

$$
\underset{\mu}{E}(x, t)=e\left[\frac{\underline{n}}{k R^{2}}+\frac{1}{c k} \frac{d}{d t^{+}}\left(\frac{\underline{n}-\underline{\beta}}{k R}\right)\right]_{\text {ret }}
$$

and

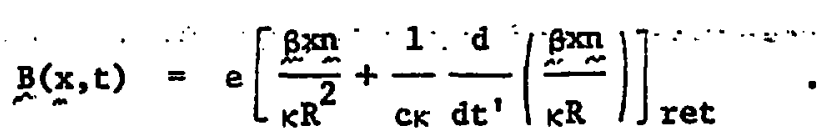

To rewrite these, three time derivatives are needed

$$
\begin{aligned}
& \frac{1}{c} \frac{d \underline{n}}{d t^{\prime}}=\frac{n \times\left(N_{n} \times \beta\right)}{R} \\
& \frac{d}{d t^{\top}} \underline{B} \equiv \underset{\sim}{\dot{B}}
\end{aligned}
$$

and

$$
\frac{1}{c} \frac{d}{d t^{\prime}}(K R)=\beta^{2}-\underset{\alpha}{\beta} \cdot \underline{\sim}-\frac{R}{c} n \cdot \dot{\beta}
$$

With these substituted into (21) we obtain

$$
E(x, t)=e\left[\frac{(\underline{n}-\underline{\beta})\left(1-\beta^{2}\right)}{k^{3} R^{2}}\right]_{\text {ret }}+\frac{e}{c}\left[\frac{n}{k^{3}} \times[(n-\beta) \times \underset{n}{\underline{\beta}}]\right]_{\text {ret }} \text {. }
$$

Of the three relativistic parameters, $\beta, K$, and $\gamma$, this may be written in terms of $\beta$ alone,

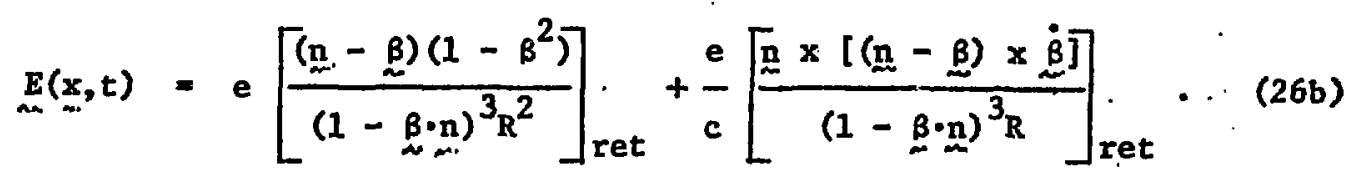


Similar substitution into (22) yields

$$
\underset{\mathbf{B}}{\mathbf{n}} \mathbf{\mathbf { n }} \underset{\sim}{\mathbf{E}}
$$

The first term, essentially static, falls off as $1 / R^{2}$ and is not of

concern to us. The acceleration term

$$
\underset{\sim}{E}(x, t)=\frac{e}{c}\left[\frac{\underline{n}}{(1-\underline{n} \cdot \underline{\underline{B}})^{3}} \times[(\underline{n}-\underline{B}) \times \underline{\underline{B}}]\right]_{\text {ret }}
$$

is of concern. If the radiation is observed from a point in the plane of

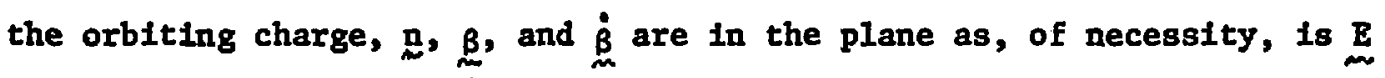
as well. This is to say that at such a point the radiation is onehundred percent polarized in the plane. The Instantaneous energy flux is given by the Poynting's vector,

$$
S=\frac{C}{4 \pi} E \times \underset{\sim}{B}=\frac{c}{4 \pi}|E|^{2} n
$$

The radial component of Poynting's vector, from Eq. (28), is

$$
\left[\underline{S} \cdot \underline{N}_{\text {ret }}=\frac{e^{2}}{4 \pi c}\left[\frac{1}{(1-\underset{N}{n} \cdot \underline{\beta})^{6} R^{2}}|\underline{n} \times[(n-\underline{\beta}) \times \dot{\beta}]|^{2}\right]_{\text {ret }}\right. \text {. }
$$

Now [S.In] ret is the power per unit area at the observation point, detected at time $t$ due to radiation emitted at time $t^{\prime}=t-R\left(t^{\prime}\right) / c$. A more useful and meaningful quantity is [S.g] ret $\left(\mathrm{dt} / \mathrm{dt}^{\prime}\right)$, the power radiated per unit area in terms of the charge's own time reference, which is the same as the power observed in the laboratory. This quantity multiplied by $R^{2}$ is the power radiated per unit solid angle: 


$$
\frac{d P}{d \Omega}=R^{2}(S \cdot n)\left(\frac{d t}{d t^{1}}\right)=(1-n \cdot \beta) \dot{R}^{2} s \cdot n
$$

Using Eqs. (28) and (29) one obtains

$$
\frac{d P}{d \Omega}=\frac{e^{2}}{4 \pi c} \frac{\operatorname{lnx}(\underline{n}-\underline{B}) \times \dot{\beta}\}\left.\right|^{2}}{(1-\underline{n} \cdot \beta)^{5}} .
$$

For circular motion, with $\underset{\sim}{B}$ taken as being in the $y$ direction and $\underset{\sim}{\dot{B}}$ in the $x$-direction and wịth $\psi$ and $\phi_{x z}$ defined $\div>$ angles between $\underline{Z}$ and $\underset{\sim}{\mathcal{B}}$ and between $\underset{\sim}{\dot{B}}$ and the projection of $\mathrm{z}$ In the $x z$ plane, respectively (FIg. 6a), we have

$$
\frac{d P}{d \Omega}=\frac{e^{2} \dot{v}^{2}}{4 \pi c^{3}} \frac{1}{(1-\beta \cos \psi)^{3}}\left[1-\frac{\sin ^{2} \psi \cos ^{2} \phi_{x z}}{\gamma^{2}(1-\beta \cos \psi)^{2}}\right] .
$$

From Eq. (33) we arrive at the root mean square angle of emission

$$
\left\langle\psi^{2}\right\rangle^{1 / 2}=1 / \gamma
$$

consistent with the angle shown in Fig. 3 for radiation at the critical

wavelength. Integrating (33) over all angles one obtains the total power

$$
P=\frac{2}{3}-\frac{e^{2} i^{2}}{c^{3}} \gamma^{4}=\frac{2}{3} \frac{e^{2} c}{\rho^{2}} \beta^{4} \gamma^{4}
$$

where $\rho$ is the orbit radius and $\dot{v}=(c \beta / \rho) v \equiv \omega v^{\prime}$, with $\omega$ the rotational frequency. In more familiar units, the power radiated by one electron is

$$
\begin{aligned}
P\left[\frac{G e^{\prime} I}{\text { sec }}\right] & =4.22 \times 10^{3} \mathrm{E}^{4} / \mathrm{p}^{2} \\
& =3.79 \mathrm{E}^{2} \mathrm{~B}^{2}
\end{aligned}
$$




$$
\underset{\sigma}{\sigma}
$$

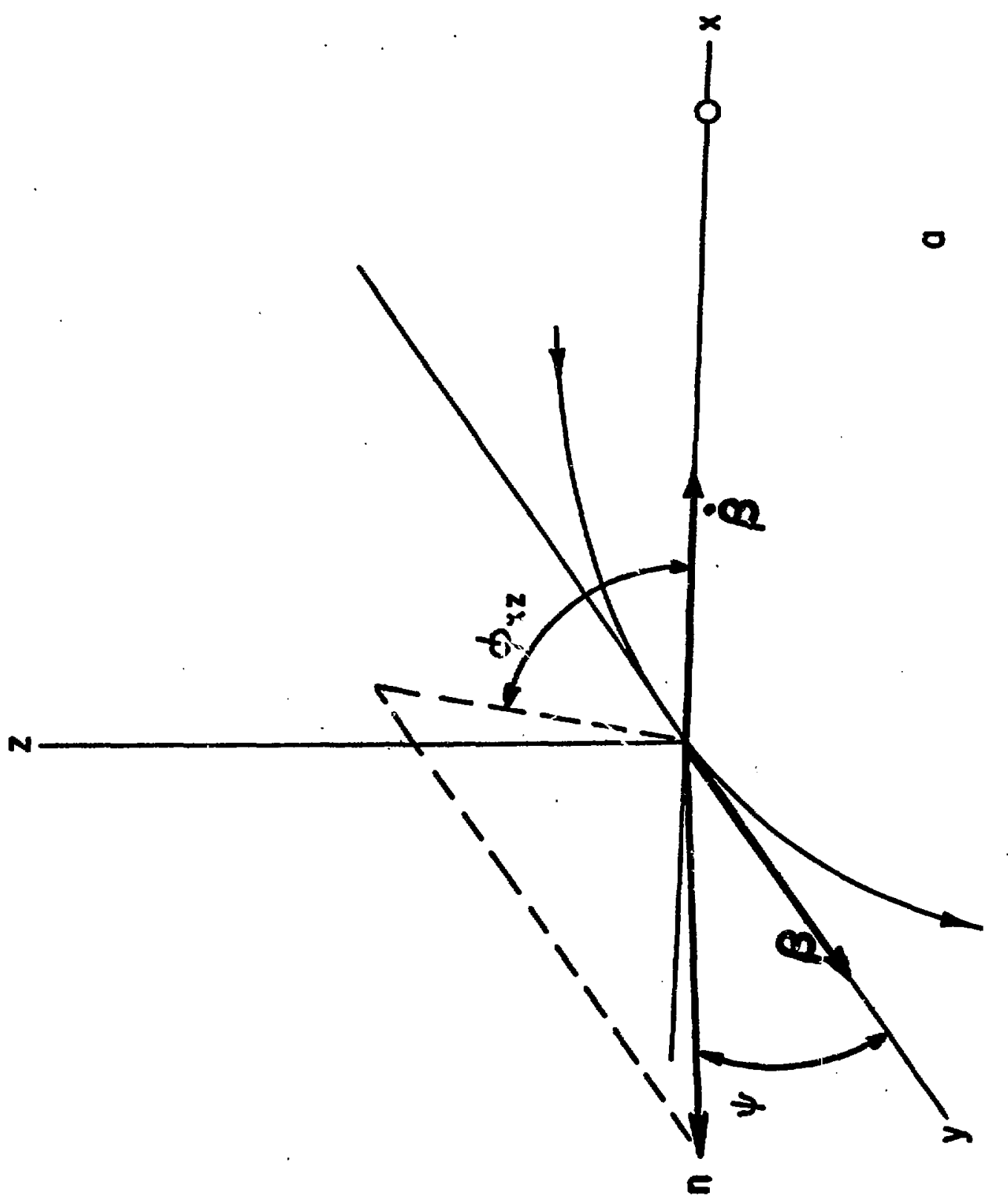




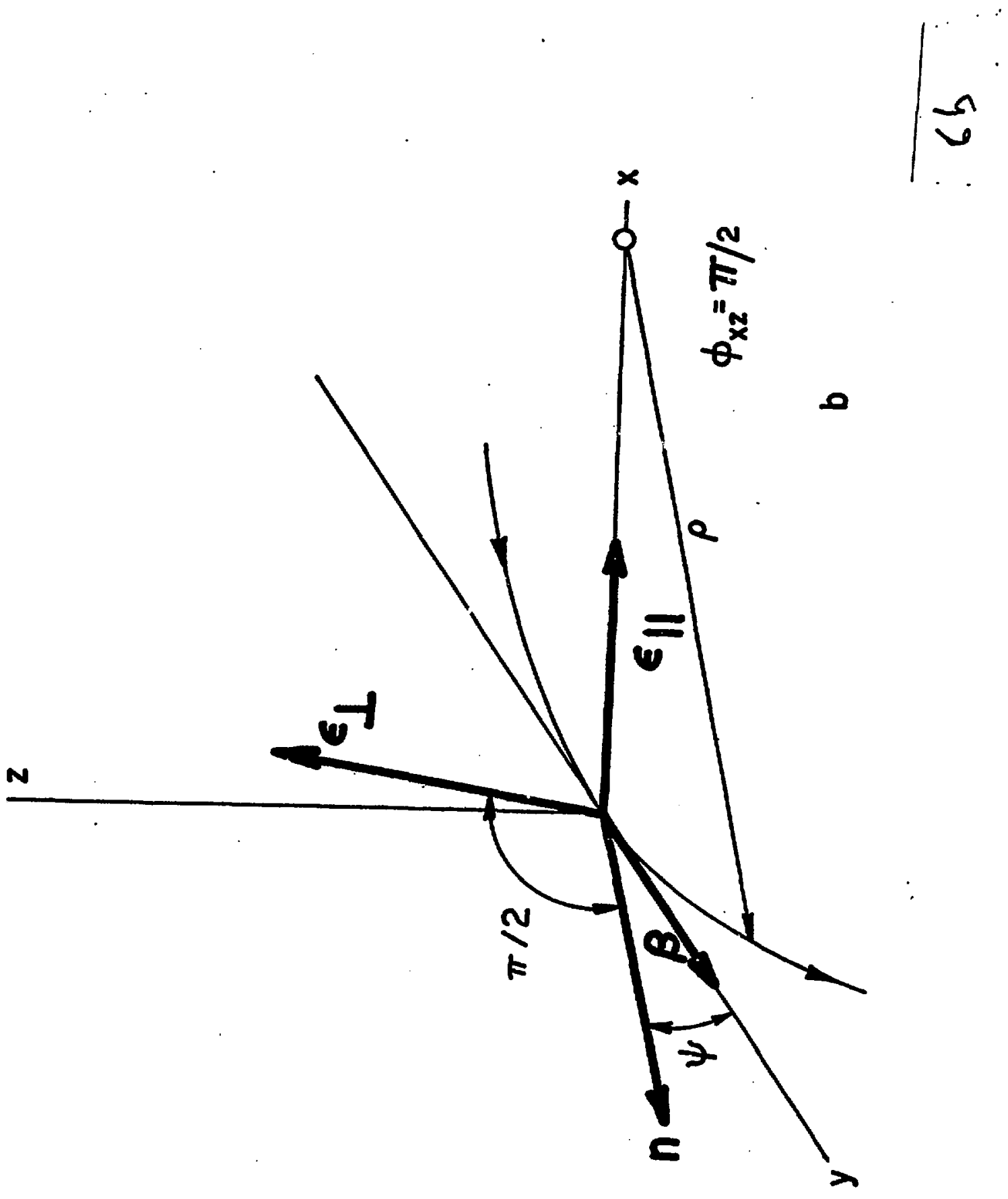


or

$$
\begin{aligned}
P(\text { watts) } & =6.76 \times 10^{-7} \mathrm{E}^{4} / \mathrm{P}^{2} \\
& =6.08 \times 10^{-10} \mathrm{E}^{2} \mathrm{~B}^{2}
\end{aligned}
$$

where $E, P$, and $B$ are in $\mathrm{GeV}$, weters and kilogauss, respectively. The radiative energy loss per turn from (35a) is then

$$
U_{0}=\frac{2 \pi \rho}{c \beta} P=\frac{4 \pi}{3} \frac{e^{2}}{p} B^{3} \gamma^{4} \text {. }
$$

For highly relativistic particles $\beta=1$ and this equation is equivalent to Eq. (4).

2.3 Classical Theory: The Frequency Characteristics of the Spectrum The frequency or wavelength distribution of the radiation is obtained by Fourier transform and Parseval's theorem [e.g. see Morse and Feshbach (1953)]. It is the frequency spectrum in the observer's frame which is of interest, and one starts with the general form for the power defined in the observer's time, $t$. Per unit solid angle this is (29)

$$
\frac{d P(t)}{d \Omega}=|A(t)|^{2}
$$

w1th

$$
A(t) \equiv\left(\frac{c}{4 \pi}\right)^{1 / 2}\left[\text { RE }_{\text {ret }}\right.
$$

Thus, the total energy so radiated over all time by a charge accelerated

during some finite time interval is

$$
\frac{d W}{d \Omega}=\int_{-\infty}^{\infty}|A(t)|^{2} d t
$$

and the spectrum may be introduced employing the transform

$$
A(\omega)=\frac{1}{\sqrt{2 \pi}} \int_{-\infty}^{\infty} A(t) e^{I_{\omega} t} d t
$$


This time integration has the effect of removing the $\phi_{x z}$ dependence from the spectrum (Fig. 6). Then, Eq. (39) becomes

$$
\frac{d W}{d \Omega}=\frac{1}{2 \pi} \int_{-\infty}^{\infty} d t \int_{-\infty}^{\infty} d \omega \int_{-\infty}^{\infty} d \omega^{\prime} A^{*}\left(\omega^{\prime}\right) \cdot A(\omega) e^{i\left(\omega^{\prime}-\omega\right) t} .
$$

Reversing the order of integration, doing the time integration first and noting that it yields the delta function $\delta\left(\omega^{r}-\omega\right)$, we obtain

$$
\frac{d W}{d \Omega}=\int_{-\infty}^{\infty}|A(\omega)|^{2} d \omega
$$

Now if

$$
\frac{d W}{d \Omega}=\int_{0}^{\infty} \frac{d I(\omega)}{d \Omega} d \omega
$$

is used to define $d I(\omega) / d \Omega$, the energy radiated per unit solid angle per unit frequency interval, for positive frequencies we have

$$
\frac{d I(\omega)}{d \Omega}=|\underline{-A}(\omega)|^{2}+|A(-\omega)|^{2} ;
$$

and since $A(\omega)=A^{*}(-\omega)$, we have

$$
\frac{d I(\omega)}{d \Omega}=2|\operatorname{si}(\omega)|^{2} .
$$

Using Eq. (28), we can obtain from (38)

$$
\begin{aligned}
& A(\omega)=\left(\frac{e^{2}}{8 \pi^{2} c}\right)^{1 / 2} \int_{-\infty}^{\infty} e^{j \omega t}\left[\frac{\underset{n}{n}[(\underline{n-\beta}) \times \dot{\beta}]}{(1-n \cdot \beta)^{3}}\right]_{r e t} d t
\end{aligned}
$$

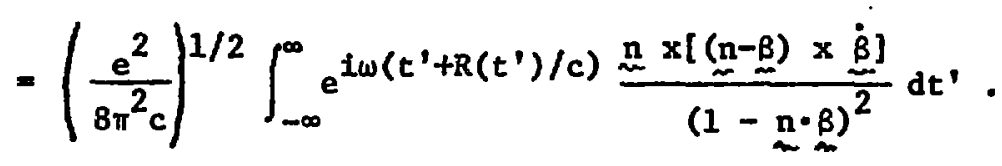

When the observation point is far from the region over which the observed emission takes place, $\underset{m}{n}$ is in effect fixed during the emission time and, in the coordinate system of Fig. $6 \mathrm{~b}$, the exponent in Eq. (45), apart from a constant phase factor, then is

$$
\left.\omega\left(t^{\prime}+\frac{R\left(t^{\prime}\right)}{c}\right)=\omega\left(t^{\prime}-\frac{\rho}{c} \sin \frac{v t^{\prime}}{\rho} \cos \psi\right)\right),
$$

where $t^{\prime}=0$ corresponds to $n$ lying exactly in the $y, z$ plane, as in Fig. $6 b$. 
Noting also that

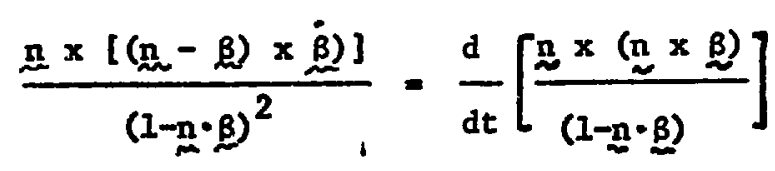

and using this to integrate Eq. (45) by parts, one obtains

$$
\begin{aligned}
\frac{d I(\omega)}{d \Omega} & =\frac{e^{2} \omega^{2}}{4 \pi^{2} c}\left|\int_{-\infty}^{\infty} \underset{n}{n} \times(n \times \underline{\beta}) e^{i \omega\left(t^{\prime}+R\left(t^{\prime}\right) / c\right)} d t^{\prime}\right|^{2} \\
\quad & \left.=\frac{e^{2} \omega^{2}}{4 \pi^{2} c} \mid \int_{-\infty}^{\infty} \underset{m}{n} \times \underset{\sim}{(n} \times \underset{\sim}{\beta}\right)\left.e^{1 \omega\left(t^{\prime}-\frac{\rho}{c} \sin \frac{v t^{\prime}}{\rho} \cos \psi\right)} d t^{\prime}\right|^{2} .
\end{aligned}
$$

The vector part of the integrand may be written; for $n$ lying in the $y, z$ plane,

$$
\underset{\sim}{n} \times(\underset{\sim}{n} \times \underset{\sim}{\beta})=\beta\left[-\varepsilon_{\mu} \sin \left(v t^{\prime} / \rho\right)+\varepsilon_{\perp} \cos \left(v t^{\prime} / \rho\right) \sin \psi\right],
$$

where $\varepsilon_{\|}$lies in the orbit plane as 1 s shown In Fig. $6 b$ and $\Sigma_{\perp}$ is normal to $\varepsilon_{\| l}$ and $\mathrm{n}_{\text {. }}$ These $\Sigma$ are the polarization directions used in Fig. 3. Defining a parameter

$$
\xi \equiv \frac{\omega p}{3 c}\left(\frac{1}{\gamma^{2}}+\psi^{2}\right)^{3 / 2}
$$

and noting that we are interested in evaluating (48) for small angles and for short times around $t^{\prime}=0$ (the time center of the light pulse in the sense of Fig. 6), integration of (48) yields

$$
\frac{d I(\omega)}{d \Omega}=\frac{e^{2}}{3 \pi^{2} c}\left(\frac{w p}{c}\right)^{2}\left(\frac{1}{\gamma^{2}}+\psi^{2}\right)^{2}\left[k_{2 / 3}^{2}(\xi)+\frac{\psi^{2}}{\left(1 / \gamma^{2}\right)+\psi^{2}} k_{1 / 3}^{2}(\xi)\right]
$$

Here the first term in the bracket is associated with radiation polarized 
$E_{11}$, the second $\varepsilon_{1} \cdot K_{2 / 3}$ and $K_{1 / 3}$ are modified Bessel functions. It may be noted that Ffg. 3 is based on Eq. (51). Evaluation of these functions for long wavelengths shows that the width of the angular distribution $\Delta \psi$ is given roughly by

$$
\Delta \psi=\frac{1}{\gamma}\left(\frac{\omega_{c}}{\omega}\right)^{1 / 3}=\frac{1}{\gamma}\left(\frac{\lambda}{\lambda_{c}}\right)^{1 / 3} \quad\left(\lambda \gg \lambda_{c}\right)
$$

whth $\omega_{c}$ and $\lambda_{c}$ as defined in Eq. (8); for short wave lengths

$$
\Delta \psi=\frac{1}{y}\left(\frac{\omega_{c}}{\omega}\right)^{1 / 2}=\frac{1}{y}\left(\frac{\lambda}{\lambda_{c}}\right)^{1 / 2}\left(\lambda \ll \lambda_{c}\right)
$$

If the angle-intensity function for the Ell $_{1}$ component, Eq. (51),

is approximated by the Gaussien $e^{-\psi^{2} / 2 \sigma_{R}^{2}}$, then $\sigma_{R}$ provides a measure of the angular spread. Such $\phi_{\mathrm{R}}$ values, given by G. K. Green (1976) for a range of photon energies and for a number of electron energies and orbit radii characteristic of accelerators used as synchrotron sources, appear in Fig. 7. 'A useful parametric! equation which is reasonably. accurate for $\lambda / \lambda_{c}=0.2$ to 100 is '

$$
\gamma \sigma_{\mathrm{R}}=0.57\left(\lambda / \lambda_{\mathrm{c}}\right)^{0.43} \text { radians. }
$$

Integration of $\mathrm{Kq}$. (5l) over angle ylelds

$$
I(\omega)=\sqrt{3} \frac{e^{2}}{c} \gamma\left(\frac{\omega}{\omega_{c}}\right) \int_{\omega / \omega}^{\infty} K_{5 / 3}(x) d x
$$

This is equivalent to Eqs. (9) and (10) though there are differences in definition: here $I(\omega)$ is the energy per turn defined for a single electron and a unit frequency range, whereas (9) is the power defined for a current 


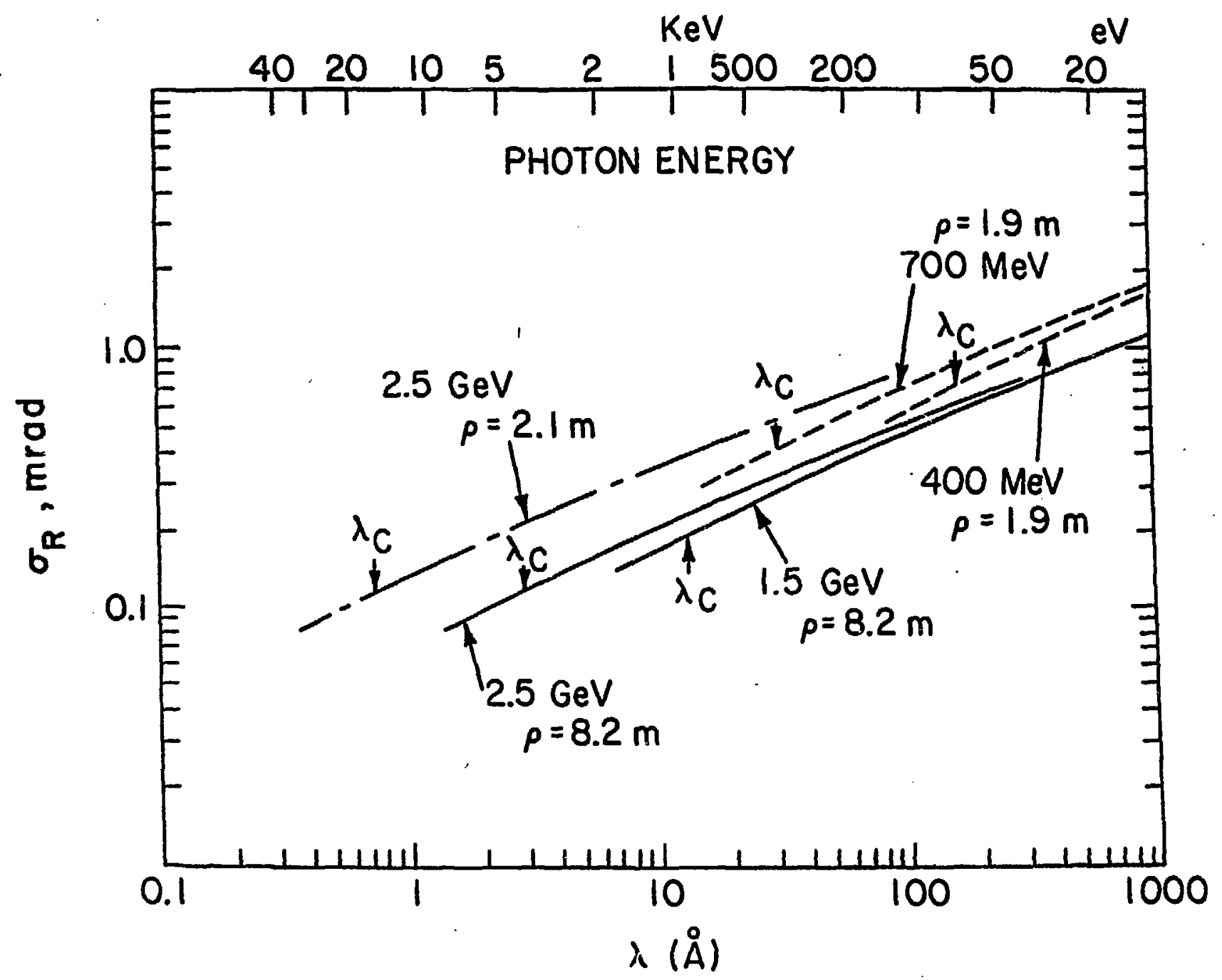


and a one-percent wavelength bandpass; Eq. (10) therefore includes: an extra factor of $1 / \lambda$. As can be seen in Fig. 1, the spectrum peaks in

the vicinity of $\omega_{c}\left(\right.$ or $\lambda_{c}$ ) and drops off sharply at higher frequencies.

This can be readily understood. The Fourfer spectrum from a single electron

should show frequencles as $\mathrm{high}$ as some approximate limit corresponding to

the reciprocal of the duration of the photon pulse seen by the observer.

As noted in Eq. (34), the rms angle of emission 1 s $21 / \gamma$ and thus the time

interval $\Delta t^{\prime}$ that light is being emitted in some given

direction is $\sim(\rho / c)(1 / \gamma)$. Since the electron is approaching the observer

at approximately the speed of light, the observation time interval is

$t_{0}=\left(\mathrm{dt} / \mathrm{dt^{ \prime }}\right) \Delta \mathrm{t}^{\prime} \cong \Delta \mathrm{t}^{\prime} / \mathrm{\gamma}^{2}$, see Eq. (15) and

the material following. Thus the inverse of this observation time, $t_{0}$, is

$$
\frac{1}{t_{0}} \sim \frac{\gamma^{3} c}{\rho}=2 / 3 \omega_{c} \text {, }
$$

which lies in the region of the peaks of Figs. 1 and 2.

The foregoing argument suggests a variant of the more usual mode for

radiation production which has been discussed by Coisson (1977).

Consider a sharp gradient in the magnetic

fleld such as that oscurring in the "fringe" region near the edge of a bending 
magnet. If there is a rapid variation in field over a length $L$ such that the time duration of electron passage through this region, $\Delta t^{\prime}=L / c$, is smaller than $\rho / c \gamma$, then this shorter time will characterize the frequency for the spectrum assosiated with this region. The observer will see a light pulse of rise time $t_{f}=\left(d t / d t^{\prime}\right) L / c \cong L / \gamma^{2} c$, so the cutoff frequency in this case is

$$
\omega_{f} \cong \frac{1}{t_{f}} \approx \frac{\gamma^{2} c}{L}
$$

Comparison of Eqs. (55) and (56),

$$
\frac{w_{f}}{w_{c}} \approx \frac{\rho}{\gamma L},
$$

shows that for normal $\rho / L$ values, of the order of 100 , with electrons having values of 1000 or more, $\omega_{f} \ll \omega_{c}$. That is, for such electrons $\omega_{f}$ does not characterize the spectrum from the fringe field region; rather it is characterized by emission from short segments along the fringe field orbit, each of length ${ }^{n} \rho_{10 c a l} / \gamma$ and emitting at $\omega_{c}$ corresponding to the local field strength. For protons at GeV energies, however, $\gamma$ is of order 1 to 10 , $\omega_{f} / \omega_{c}$ is of order 10 to 100 , and the fringe region emits a spectrum characterized by $\omega_{F}$. Fringe field radiation, from protons at much shorter wavelength! than that pioduced by the bending magnet fields, has indeed been observed at CERN. 
A different spectrum is produced in an undulator, a

region of orbit along which the magnetic field, though static in time,

consists of wany short segments successively alternating in direction. If

the length of one period is $\lambda_{0}$, then, in the electron's coordinate system,

the particle is subject to an alternating field of periodicity $\lambda_{0} / \gamma_{\circ}$ In

its frame the electron emits at this wavelength and the observer in his

laboratory frame sees a wavelength

$$
\lambda \sim \lambda \lambda_{0} / \gamma^{2}
$$

This argument is only qualitative and the actual situation is more complex.

A detalled discussion of the undulator spectrum is given in sect. 6 . 


\section{ELECTRON OPTICS AND STORAGE RINGS}

\subsection{Introduction}

An electron storage ring is an assemblage of many components, as is

illustrated in Fig. 8. There is an ultra high vacuum chamber in which the

electrons circulate, with ports at the locations of bending magnets so that

the synchrotron light may be utilized. There are magnets, which provide

fields with gradients to act as lenses of the electron optics system.

Focusing and bending functions have sometimes been combined in magnet

structures. To bring electrons into the ring there is an infection

system usually designed to operate at a lower energy than the maximum for the ring

itself, and there must be one or more radio frequency (KF) cavities to accelerate

electrons to full energy and to replace the energy lost to synchrotron

radiation. There will, in general, be provision for dumping the electron

beam at the end of an operating run and such a dump arrangement may be used

also to collect those electrons which have strayed too far from the ideal

orbit to be maintained in circulation. There are vacuum pumps and gauges,

magnet correction coils, and beam detection devices for diagnostic measurement

and control. There may be incorporated, as part of the orbit design, straight 


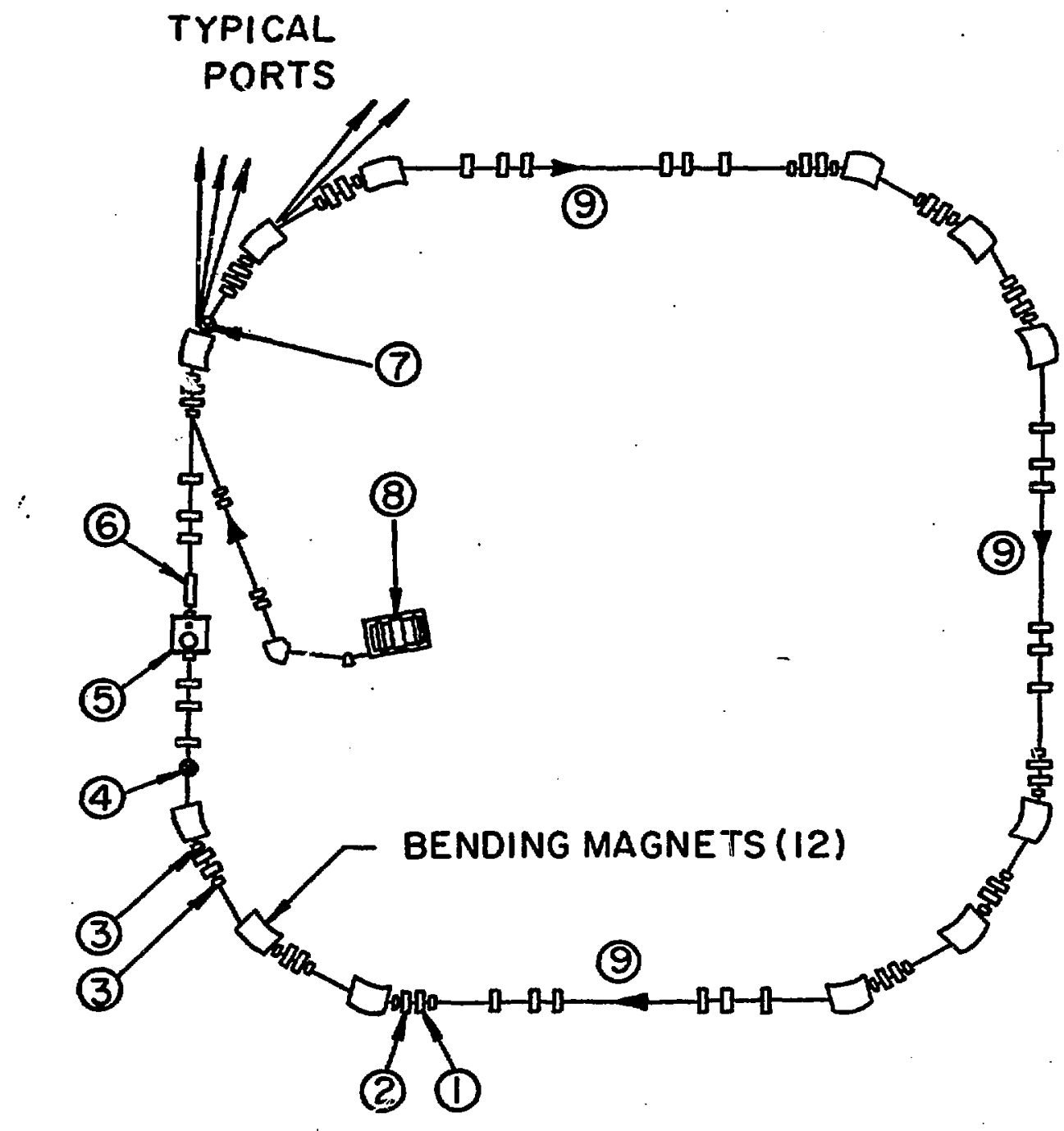

I FOCUSSING QUADRUPOLE 6 VERTICAL EXTRACTION

2 DEFOCUSSING QUADRUPOLE MAGNET

3 SEXTUPOLE CORRECTIONS 7 INJECTION KICKER

4 EXTRACTION KICKER

MAGNET

5 ACCELERATING CAVITY $8100 \mathrm{MeV}$ MICROTRON

9 STRAIGHT SECTIONS (AVAILABLE FOR WIGGLERS)

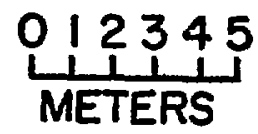


sections unencumbered by other hardware, into which any of several classes of wiggler or undulator magnets may be placed. The result is a complex device. As a practical matter, this hardware and the radiation shielding which almost invariably surrounds a ring of GeV range Inhibit access of the experimenter to the regions from which Itght is emitted.

The purpose of this section is to consider the electron beam stored

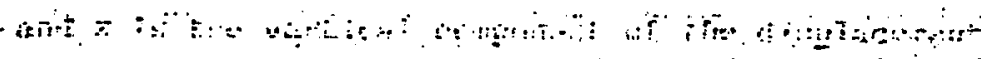
In such a ring for it is the electron beam optics which determine the brightness, the intensity, and the time structure of the photon source. For some given set of magnet currents, an electron having momentum $p_{0}$ under certain initial conditions is confined to a particular equilibrium orbit. In the absence of inevitable magnetic field defects, caused by various construction imperfections, this "Ideal" or "reference" orbit lies In a horizontal plane so located that everywhere the field is vertical. The energy of an electron lying in this orbit is defined to be $\mathrm{E}_{0^{*}}$ In practice few electrons move on the reference orbit. This is due to errors in electron position and momentum upon injection, to imperfections and misalignment of storage ring hardware, to electron-electron and other scattering processes, and to the fact that synchrotron 1 ight is being emitted, that emission being a quantum process. 
It is convenient to utilize a coordinate system related to the reference orbit. The position of a point $P$ near the reference orbit is specified by $(s, x, z)$. The azimuthal coordinate $s$ equals the arc length along the ideal orbit measured from some fixed reference point to that point on the orbit closest to the point $P ; X$ is the horizontal radial component of the displacement of $\mathbf{P}$ from the ideal orbit (positive in the outward direction); and $z$ is the vertical component of the displacement, see

Fig. 9. We will be describing the electron beam in terms of $x$ and $z$ and their derivatives,

$$
x^{\prime} \equiv \frac{d x}{d s} \text { and } z^{\prime} \equiv \frac{d z}{d s}
$$

obviously, the size of the photon source depends on the distribution in $x$ and $z$; the angle of light emission depends on $x^{\prime}$ and $z^{\prime}$ as well as on the properties, already discussed, of the natural cone into which synchrotron light is emitted.

This section is devoted to the description of the motion of the electrons in a storage ring; included are the effects produced by magnet lattice imperfections and the consequences for orbit behavior of light emission by the electrons. It will be seen that the magnet lattices 


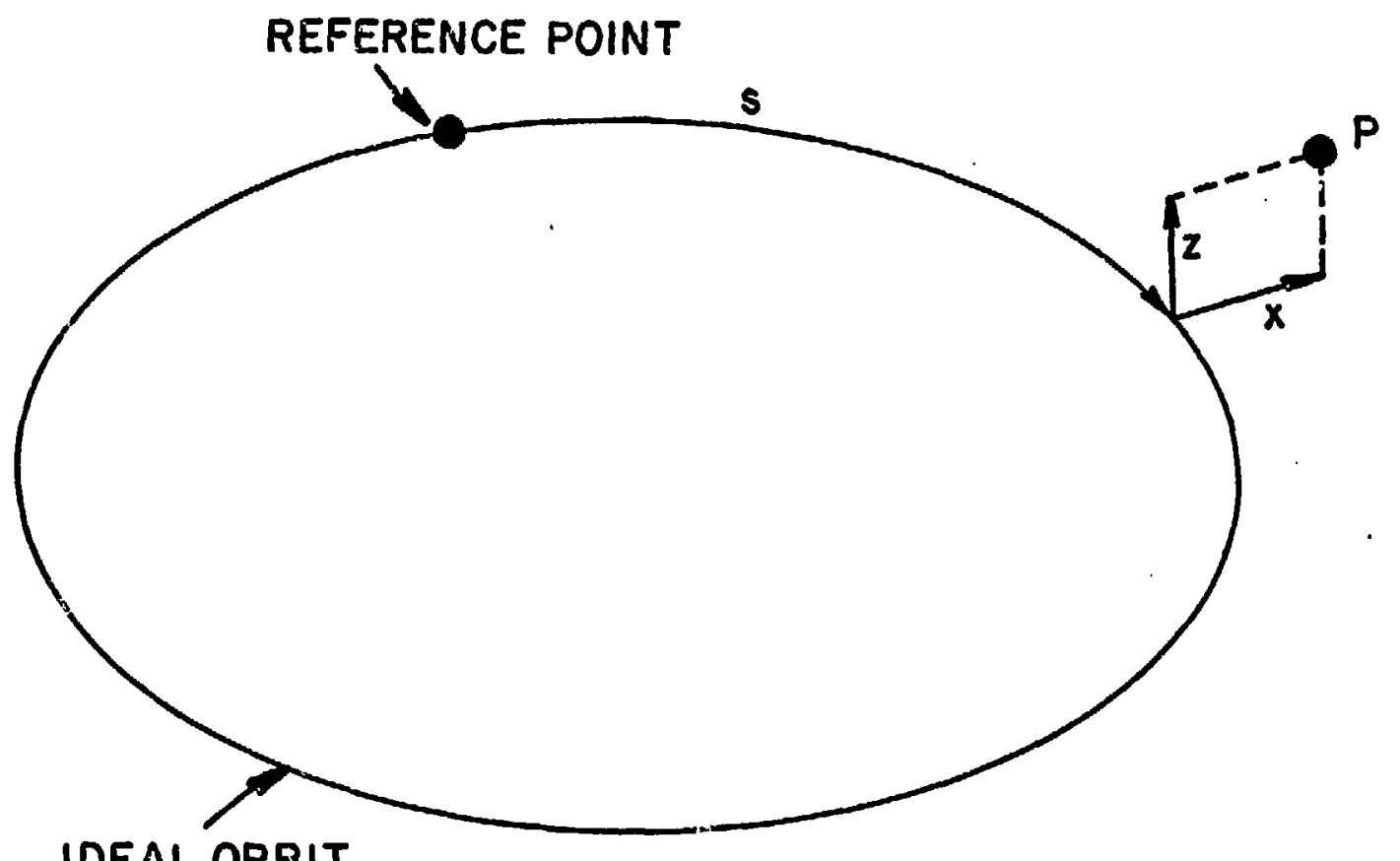

IDEAL ORBIT 
usually incorporate "lenses" of a type not normally encountered in photon optics, quadrupole magnets which are focusing in either the $z$ or $x$ coordinate but are defocusing in the other transverse coordinate, $x$ or $z$ respectively. In order to obtain stable transverse "betatron" oscillation in both the horizontal and the vertical coordinates, the quadrupole magnets are arranged in an alternating sequence: focusing, defocusing, focusing, .... This alternating gradient principle [Courant and Snyder (1958)] is fundamental to the remarkable advance in accelerator technology over the past twenty-five years.

$\because \therefore \quad$ Energy lost by electrons as synchrotron radiatton is restored when they pass through the radio frequency cavity. An electron of the correct energy travelling on the ideal orlit interacts synchronously with the cavity just to restore the loss, while non-ideal particles may be set into longitudinal "synchrotron" and transverse "betatron" osciliation or may even be lost from the beam. Because synchrotron radiation is given off as discrete photons, the electrons are subject to quantum fluctuations. Since the photon emission can be described as a stochastic process, we shall see that the emission determines the equilibrium energy distribution, the length of the electron bunches, and the horizontal and vertical dimensions of the beam. The stochastic character of the emfssion leads to eleatron phase space ( $x-x^{\prime}$, $\left.z-z^{\prime}\right)$ distributions having Gaussian form.

These phase space distributions convoluted with the geometry naturally characteristic of the synchrotron cadiation emission process together determine the spatial properties of the light "source". In general, the angles $x^{\prime}$ and $z^{\prime}$ are sulaller than the natural emission cone angle. The spatial widths of the electron.distribution are denoted $\sigma_{x}$ and $\sigma_{z}$ and the angular widths $\sigma_{x}$ and $\sigma_{z^{\prime}}$. The widths vary around the ring according to 


$$
\sigma_{x}(s)=\sqrt{\varepsilon_{x}^{\beta} \beta_{x}(s)} \text { and } \sigma_{z}(s)=\sqrt{\varepsilon_{z}^{\beta} \beta_{z}(s)} \text {, }
$$

where $\beta_{X}$ and $\beta_{z}$, the "amplitude functions", are determined by the focusing properties of the magnetic lattice, and $\varepsilon_{x}$ and $\varepsilon_{z}$, the emittances of the electron beam, do not depend on the azimuthal coordinate s. The emittances define an area in the $x-x^{\prime}$ plane and, independently, one in the z-z' plane, each of which encompasses a specifled fraction (say one standard deviation) of the electrons. At those azimuthal locations in the orbit where $\beta_{x}^{\prime}=0$, the area in $x-x^{\prime}$ phase space is an upright ellipse with its axes along $x$ and $x^{\prime}$, and $\sigma_{x^{\sigma}} \sigma^{\prime}=\varepsilon_{x^{*}}$ In transposition to other locations around the azimuth the ellipse maintains constant area, but becomes t1lted and changes its proportions. Therefore, around the orbit the electron beam varies in its geometrical characteristics as a light source. Deviations from ideality of the magnetic field, si:ch as those due to magnet misalignment or to errors in magnet fabrication, have adverse effects on orbit characteristics. For example, they act to couple the horizontal and vertical oscillation modes and, in so doing, they constitute the principle factor limiting the extent to which the vertical size of the electron beam can be squeezed.

The tune values $v_{x}$ and $v_{z}$, which are the number of horizontal and vertical "betatron" oscillations executed by an electron in one revolution, are parameters of fundamental importance in minimizing the effects due to magnetic field imperfections. As is the case in, say, the mechanics of a rotating machine, not all defects in construction are equaliy harmful. Those that have the most deleterious effects are those that drive resonant modes. Similarly, it is resonance phenomena that determine the performance of the storage ring, and it is the choice of the tunes $v_{x}$ and $v_{z}$ which allows one 
to avold resonanres. As one might expect, Integer and half integer values of the tunes must be avoided. Detailed study of the effects of magnetic Imperfection deterwines which regions of tune values are allowed and appropriate for satisfactory operation of the storage ring.

Another function which plays an important role in the description of the electron optics is the dispersion function $n(s)$. It is a measure of the sensitivity of the position of the equilibrium orbit to small deviations of the electron momentum from the ideal value $p_{0_{0}}$ If the electron beam has a fractional momentum spread $\Delta \mathrm{p} / \mathrm{p}_{\mathrm{o}}$, then the radial beam size is

$$
\left\{E_{x^{\beta}}(s)+\left[n(s) \frac{\Delta_{p}}{p_{0}}\right]^{2}\right\}^{1 / 2} .
$$

In summary, let us collect a few of the functions which will be used in describing the optics of the electron beam:

$$
\begin{aligned}
& \beta_{x}(s), B_{z}(s) \text {, amplitude functions; } \\
& E_{x}, \varepsilon_{z} \text {, emittances; } \\
& \nu_{x}, \nu_{z}, \text { tunes; } \\
& \text { and } \quad n(s), \text { dispersion function. }
\end{aligned}
$$

Also, in an effort to prepare the reader for what follows in our discussion of the electron optics, we note that when dealing with the equations of motion, it is convenient to utilize trangfer matrices to describe the evolution of electron trajectories as a particle traverses the orbit azimuth. Consider an electron in a "drift space" region free of magnetic fields. If its coordinates are $x(0)$ and $x^{\prime}(0)$ at some point, then at a distance $\ell$ farther along, it has coordinates $x(\ell)=x(0)+\ell x^{\prime}(0)$ and $x^{\prime}(l)=x^{\prime}(0)$. This can be described by 


$$
\left(\begin{array}{l}
x(l) \\
x^{\prime}(l)
\end{array}\right)=\left[\begin{array}{ll}
1 & l \\
0 & 1
\end{array}\right]\left(\begin{array}{l}
x(0) \\
x^{\prime}(0)
\end{array}\right)
$$

where the square bracket 1E the transfer matrix. It may be noted in passing that this same scheme will be used to describe photon optics.

This Section is organized as follows. We first consider the motion of an electron subject to the magnetic focusing forces - betatron oscillations. Then the effect of the RF cavity is discussed - gynchrotron oscillations. Next we describe the way in which the equilibrium transverse beam sizes, the bunch length, and the energy spread are determined from the quantum fluctuations associated with the photon emission. This is followed by a discussion of the Touschek effect, electron-electron scattering within a bunch which causes loss of particles from it. Such an interaction has a rate depending on the square of the electron density, and the Touschek beam Iffetime therefore varies inversely with the electron density. At low energies, the Touschek effect tends to be dominant among the factors determining the length of time that beam may be stored. Increase in stored current (high photon flux), decrease in beam cross section (high source "brightness"), and decrease in electron bunch length (short light pulse duration) - three characteristics which separately or in combination are required for many experiments - all are associated with high eiectron density and hence with the effects of increased electron-electron interaction: namely spatial spreading of the bunch and loss of electrons from 1 .

The section concludes with a list of the parameters appropriate to one storage ring as an illustration.

The material discussed in the first part of this section is considered in greater detail in Courant and Snyder (1958) and Steffen (1965), while the 
material in the latter half benefits from Robinson (1958) and Sands (1970). Sands (1970) and Bruck (1966) are excellent general references.

\subsection{Equations of Motion}

To define the system of coordinates for the equations of motion, we first specify an origin and let $\underline{R}(s)$ be the radius vector from the orfgin to a point on the ideal orbit a distance $s$ from the reference point. Denoting the derivative $\mathrm{d} / \mathrm{ds}$ by a prime, the unit vector tangent to the reference orbit is

$$
\mathrm{e}_{s}(s)=\underline{R}^{\prime}(s) \text {, }
$$

and the unit vector in the horizontal plane normal to the ideal orbit is

$$
\underline{e}_{x}(s)=\rho(s) \underline{R}^{\prime}(s),
$$

where $\rho(s)$ is the local radius of curvature. The unit vector in the vertical

direction is

$$
e_{z}=\underset{m s}{e}(s) \times \underset{m x}{e}(s)
$$

and one sees that

$$
\underset{j}{e}(s)=-G(s) \underset{s x}{e}(s) \text { and } \underset{i m x}{e}(s)=G(s) \underset{m s}{e}(s),
$$

where

$$
G(s)=1 / 0(s) \text {. }
$$

The radius vector of an electron near the reference orbit. and its

first time derlvative are given by

$$
\underline{r}=\underline{R}+x \stackrel{e}{x}_{x}+\underline{e}_{z}
$$


and $\underline{v}=\underline{\dot{r}}=w[1+G:(s) x] e_{-8}+\dot{x} e_{x}+\dot{z} e_{z}$,

where $w=\dot{s}$ is the speed of the electron projected along the ideal orbit.

It follows that

$$
v^{2}=w^{2}[1+G(s) x]^{2}+\dot{x}^{2}+\dot{z}^{2}
$$

Keeping only first-order cerms,

$$
\frac{d \ell}{d s}=\frac{v}{w} \cong 1+G \cdot(s) x \text {, }
$$

where in time dt the eisctron travels g-distance dl along th trajectory while its projection on the reference orbit travels a distance ds (see Fig. 10)..

The Lorentz force equations (3) imply

$$
\begin{aligned}
y-w^{2} G(s)[1+G(s) x] & =\frac{e}{\gamma m c}\left[{ }_{B}-w[1+G(s) x] B_{z}\right], \\
\ddot{z} & =\frac{e}{\gamma m c}\left[z[1+G(s) x] B_{x}-\dot{x B}_{s}\right],
\end{aligned}
$$

and the invariance of the magnitude of the velocity v. Evaluating (66a)

on the ideal orbit $\left(x=x^{\prime}=z=z^{\prime}=0\right)$ with $w=v$, we find

$$
G(B)=\left.\frac{e}{P_{0} c} B_{2}\right|_{X=2=0},
$$

where $p_{0}$ is the momentum of the electron on the ideal orbit. In order to linearize the Lorentz equations $(66 a, b)$ about the reference orbit, one approximates the magnetic field in its vicinity by 


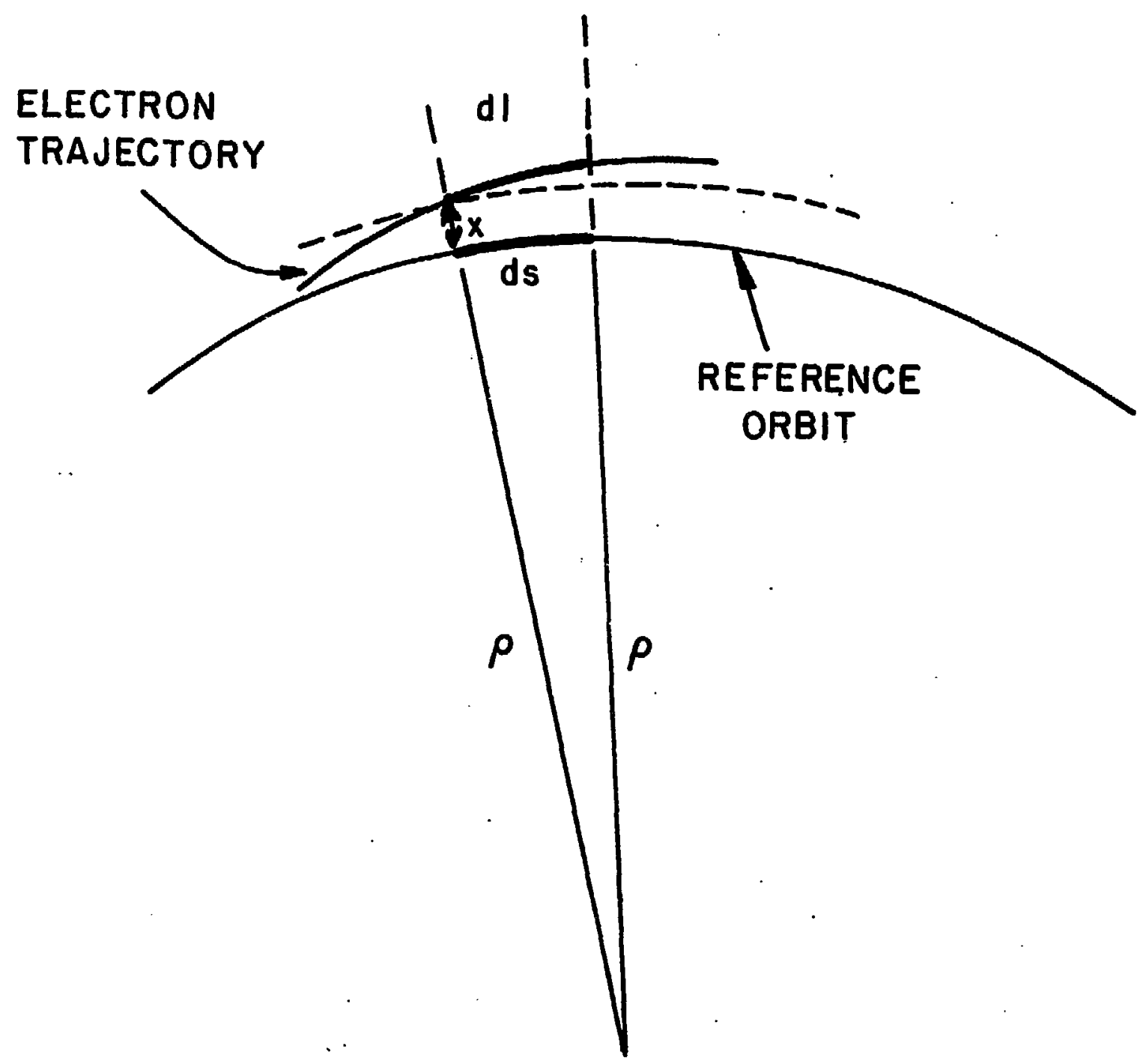




$$
\begin{aligned}
\frac{e}{P_{0} c} B_{z} & \cong G(s)+K(s) x, \\
\frac{e}{p_{0} c} B_{x} & \cong K(s) z, \\
B_{s} & \cong 0,
\end{aligned}
$$

where the focusing function

$$
K(s)=\left.\frac{e}{p_{0} c} \frac{\partial z}{\partial x}\right|_{x=z=0} .
$$

Keeping only first-order terms, we obtain the trajectory equations

$$
\begin{gathered}
z^{\prime}-k(s) z=0 \\
=0
\end{gathered}
$$

here $\Delta p=p-p_{0}$, and we use the first-order approximations (65) and $z \simeq w^{2} z^{\prime}$.

From Eqs. $\left(70_{a}, b\right)$ we see immediately the need for alternating gradients to obtain strong focusing, since a gradient $K$ having the proper sign to be focusing for the radial (x) displacements is defocusing for the vertical (z) oscillations. Defining the field gradient index

$$
n(s)=-K(s) / G^{2}(s) \text {, }
$$

we see that if $n(s)=n$, independent of $s$, then one obtains stable $x$ and $z$ oscillations only if

$$
0<n<1
$$




\subsection{Betatron Oscillations, Dispersion Function, Momentum Compaction}

The specific array of magnetic flelds around the storage ring, referred to as the magnetic lattice, enters Eqs. (70a,b) through the functions $G(s)$ and $K(s)$, where $G(s)$ is proportional to the dipole component of the magnetic field and $K(s)$ to the quadrupole component. The proportionality constant is the inverse of the magnetic rigidity of the electron of energy $E_{0}$,

$$
B(s) / G(s)=B(s) \rho(s)=p c / e=33.35 \mathrm{E}(\mathrm{GeV}) \text {, kgauss }-\mathrm{m} \text {. }
$$

Clearly $G(s)$ and $K(s)$ are perlodic,

$$
G(s+L)=G(s) \text { and } K(s+L)=K(s)
$$

where $L$ is the total Iength or "clrcumference" of the reference orbit. It is conventional to define the dispersion function $\eta(s)$ as the periodic solution to

$$
\begin{aligned}
n^{\prime}+\left[K(s)+G^{2}(s)\right] n & =G(s), \\
n(s+L) & =n(s),
\end{aligned}
$$

80 that the solution to the radial equation $(70 \mathrm{a})$ can be written

$$
x(s)=x_{e}(s)+n(s) \frac{\Delta p}{p}
$$

The term $n(s) \Delta_{p} / p$ arises because the equilibrium orbit (often called the closed orbit) for an electron of momentum $P_{0}+\Delta p$ differs from the reference orbit corresponding to $\Delta \mathrm{p}=0$. Transverse oscillations in $\mathrm{x}$ about this. closed orbit are represented by $x_{B}$, a non-perlodic solution of

$$
x_{\beta}^{\prime \prime}+\left[K(s)+G^{2}(s)\right] x_{\beta}=0
$$

which depends on the initial conditions. The transverse modes described by (74) 
as well as by $(70 \mathrm{~b})$ are called betatron oscillations. In what follows we shall drop the subscript " $\beta$ " when referring to radial deviation from the closed orbit.

The circumference of the closed orbit depends on the momentum deviation, and one defines the momentum compaction $\alpha$ by

$$
\frac{\Delta L}{\mathrm{~L}}=\alpha \frac{\Delta \mathrm{p}}{\mathrm{p}}
$$

With Eq. (65.) we can obtain $\alpha$ in terms of the dispersion function,

$$
\alpha=\frac{1}{\mathrm{~L}} \int_{0}^{\mathrm{L}} \mathrm{ds} n(s) G(s) .
$$

3.4 Solution of Hill's Equation

The relations describing the betatron oscillations have the form of

Hill's equation

$$
\begin{aligned}
\frac{d^{2} y}{d s^{2}}+k(s) y & =0, \quad y=x \text { or } z \\
k(s+L) & =k(s) ;
\end{aligned}
$$

here $y$ represents efther an $x$ or $a z$ displacement from the ideal orbit. A

solution is facilitated by change of variables. We introduce the phase

$$
\phi=\frac{1}{v_{y}} \int_{0}^{s} \frac{d \tilde{s}}{\beta_{y}(\tilde{s})}
$$

where the tune $v$ is defined by

$$
2 \pi v_{y}=\int_{0}^{L} \frac{d s}{\beta_{y}(s)} \quad y=x \text { or } z
$$


and $\beta_{y}(s)$, the amplitude function, will be determined shortly. Normalizing the displacement by the square root of the amplitude function,

$$
\xi=B^{-1 / 2} y \text {, }
$$

one easily shows that

$$
\frac{1}{v} \frac{d \xi}{d \phi}=\beta^{-1 / 2}\left(\beta y^{\prime}-1 / 2 \beta^{\prime} y\right),
$$

again with a prime to denote d/ds. If $\beta(s+L)=\beta(s)$ and if $\beta(s)$ satisfies

the relation

$$
\frac{1}{2} \beta \beta^{\prime \prime}-\frac{1}{4} \beta^{\prime 2}+k(s) \beta^{2}=1 \text {, }
$$

Eq. (77) becomes

$$
\frac{d^{2} \xi}{d \phi}+\nu^{2} \xi=0,
$$

the equation of a harmonic oscillator.

The Courant-Snyder invariant, a first-integral of the motion, is

$$
\varepsilon_{y}=\xi^{2}+\left(\frac{1}{\nu} \frac{d \xi}{d \phi}\right)^{2}
$$

Introducing the perlodic functions called the Twlss parameters,

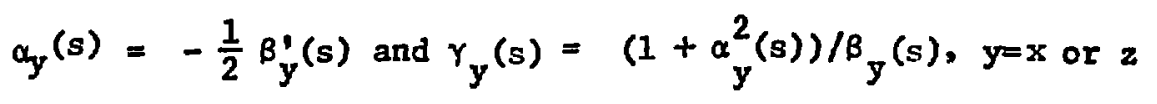

and using (78c) and (79), we find the Courant-Snyder invariant to be

$$
\varepsilon_{y}=\gamma_{y}(s) y^{2}+2 \alpha_{y}(s) y y^{\prime}+\beta_{y}(s) y^{\prime 2}, y=x \text { or } z \text {. }
$$

We stress that, although $\alpha_{y}, \beta_{y}, \gamma_{y}$, and $y$ depend on the azimuthal coordinate $s$, 
the invariant $\varepsilon_{y}$ does not, This invariant $\varepsilon_{y}$, the emfttance, constitutes an ellipse in phase space which defines the position and trajectory angle of the electron in its betatron oscillations.

The general solution of (77) for the betatron osclilations can be

written

$$
y(s)=\sqrt{\varepsilon_{y} \beta_{y}(s)} \cos \left[\int_{0}^{s} \frac{d \tilde{s}}{\beta_{y}(\hat{s})}+\delta\right],
$$

with $\varepsilon_{y}$ and $\delta$ depending on the inttial condition $y_{0}$ and $y_{0}$ The argument;

$\psi_{y}(s)$, of the cosine changes by $2 \pi v$ after each revolution. Differentiating,

we obtain

$$
y^{\prime}(s)=-\sqrt{\varepsilon_{y} / \beta_{y}(s)}\left[\sin \psi_{y}(s)+\alpha_{y}(s) \cos \psi_{y}(s)\right] .
$$

\subsection{The Phase Ellipse}

On each revolution around the ring, as a given electron passes a

particular azimuthal location, the corresponding values of $y$ and $y^{\prime}$ vary, but they always 1le on the phase ellipse (84). Again we let y represent either the horizontal coordinate $x$ or the vertical $z$. Although the shape and orientation of the ellipse change from point to point around the ring, the area remains invariant at the value $\pi \varepsilon_{y}$. In Fig. 11 we illustrate a phase ellipse and four points at extrema and intercepts. The coordirates 


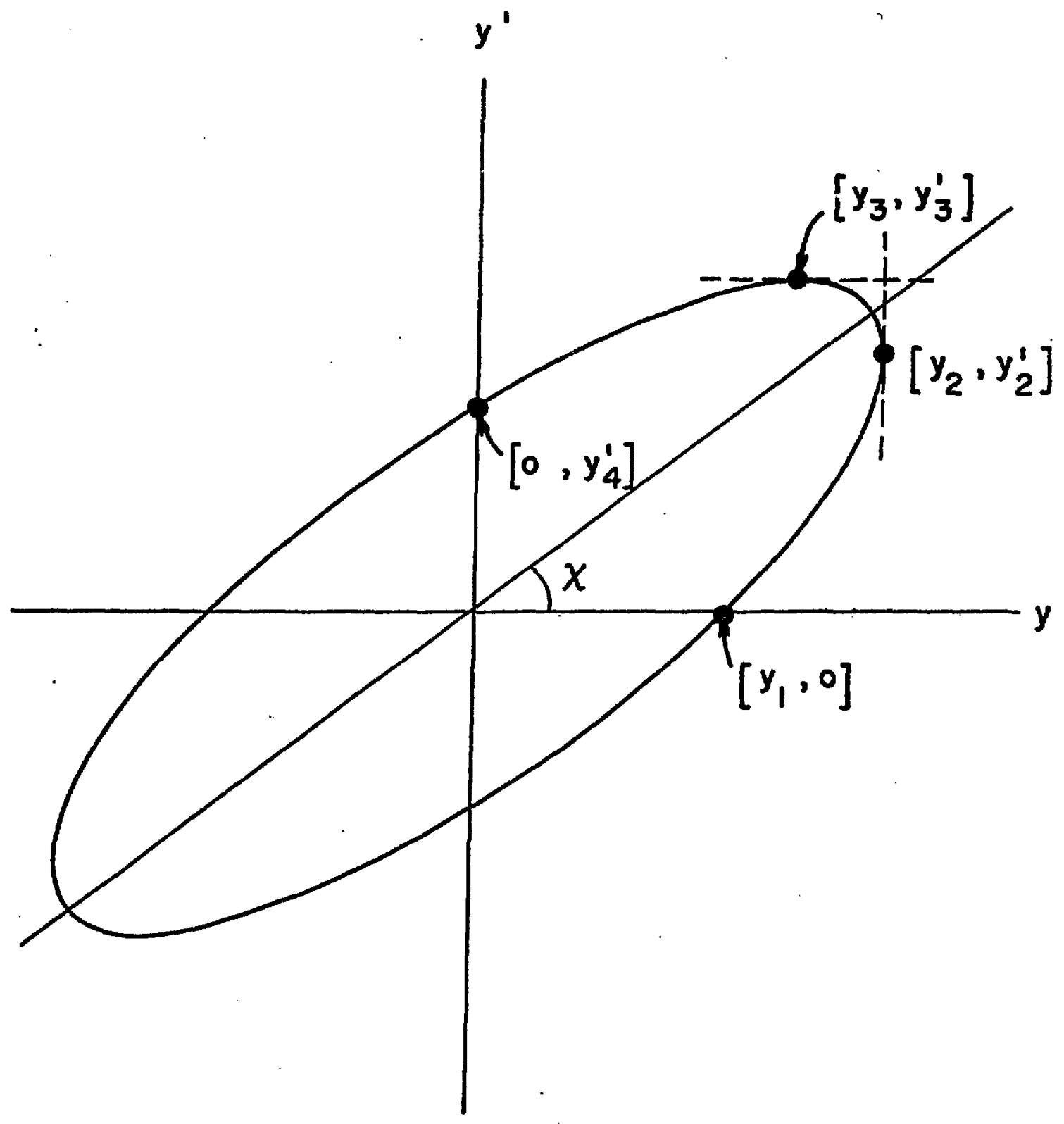




$$
\begin{aligned}
& y_{1}=\sqrt{\varepsilon_{y} / \gamma_{y}}, y_{1}^{\prime}=0, \\
& y_{2}=\sqrt{\beta_{y} \varepsilon_{y}}, y_{2}^{\prime}=-\alpha_{y} \sqrt{\varepsilon_{y} / \beta}, \\
& y_{3}=-\alpha_{y} \sqrt{\varepsilon_{y} / \gamma_{y}}, y_{3}^{\prime}=\sqrt{\varepsilon_{y} \gamma_{y}}, \\
& y_{4}=0, y_{4}^{\prime}=\sqrt{\varepsilon_{y}^{\prime} / \beta_{y}},
\end{aligned}
$$

and the tilt angle $X$ of the ellipse is given by

$$
\tan 2 x=-2 \alpha_{y} /\left(\beta_{y}-\gamma_{y}\right)
$$

An upright ellipse, which corresponds to $q_{y}=-\beta_{y}^{\prime} / 2=0[E q$. (83) ],

occurs at locations in the ring where the amplitude function takes its

extrema values. At a location where $\alpha_{y}=0$,

$$
y_{\max }=\sqrt{E_{y}^{\beta} \beta_{y}} \text { and } y_{\max }^{\prime}=\sqrt{E_{y} / \beta_{y}} \text {. }
$$

Hence, the maximum displacement anywhere in the ring occurs at the location

of the $\beta_{\max }$, and the maximum angular deviation occurs at the position of the $B_{\min }{ }^{*}$

3.6 The Transfer Matrix

Let $\approx\left(s / s_{0}\right)$ be the "cosine-like" snlution of (77)' satisfying the initial conditions $c\left(s_{0} / s_{0}\right)=1$ and $c^{\prime}\left(s_{0} / s_{0}\right)=0$; and let $s\left(s / s_{0}\right.$ be the "sine-11ke" solution satisfying $S\left(s_{0} / s_{0}\right)=0$ and $s^{\prime}\left(s_{0} / s_{0}\right)=1$. Since (77) is a linear equation, an arbitrary solution can be related to its infial 
values by

$$
\begin{aligned}
& y(s)=C\left(s / s_{0}\right) y\left(s_{0}\right)+s\left(s / s_{0}\right) y^{\prime}\left(s_{0}\right) \\
& y^{\prime}(s)=C^{\prime}\left(s / s_{0}\right) y\left(s_{0}\right)+s^{\prime}\left(s / s_{0}\right) y^{\prime}\left(s_{0}\right) .
\end{aligned}
$$

This is conveniently written as a matrix equation,

$$
\left[\begin{array}{c}
y\left(s_{2}\right) \\
Y^{\prime}\left(s_{2}\right)
\end{array}\right]=\underline{M}\left(s_{2} / s_{1}\right)\left[\begin{array}{l}
Y\left(s_{1}\right) \\
y^{\prime}\left(s_{1}\right)
\end{array}\right],
$$

where $\underline{M}\left(s_{2} / s_{1}\right)$ is called the transfer matrix between $s_{1}$ and $s_{2}{ }^{*}$. It follows from (77) that $c\left(s / s_{0}\right) s^{\prime}\left(s / s_{0}\right)-c^{\prime}\left(s / s_{0}\right) s\left(s / s_{0}\right)=1$ for any $s$, so the determinant of the transfer matrix is always unity. Of particular interest is the transfer matrix $\underline{M}(s+L / s)$ corresponding to one revolution; using (85) and (86) we see that

$$
\underline{M}(s+L / s)=\left[\begin{array}{lc}
\cos 2 \pi \nu_{y}+\alpha_{y}(s) \sin 2 \pi \nu_{y} & \beta_{y}(s) \sin 2 \pi v_{y} \\
-\gamma_{y}(s) \sin 2 \pi \nu_{y} & \operatorname{sos} 2 \pi v_{y}-\alpha_{y}(s) \sin 2 \pi v_{y}
\end{array}\right]
$$

The quantity $v_{y}$, the tune value introduced in $(78 \mathrm{~b})$, enual.s

the number of betatron oscillations per revolution. The trace of this matrix

is $2 \cos 2 \pi v_{y}$ independent of the starting point $s$, and its determinant is unity.

A storage ring is generally constructed of magnetic segments in each

of which the focusing function has the same value. A focusing quadrupole of

length $\ell$ and gradient $g=\mathrm{dB}_{2} / \mathrm{dx}$ operating on an electron with magnetic 
rigidity $B \rho$ has the transfer matrix

$$
\left[\begin{array}{ll}
\cos \ell \sqrt{K} & \frac{1}{\sqrt{K}} \sin \ell \sqrt{k} \\
-\sqrt{K} \sin \ell \sqrt{K} & \cos \ell \sqrt{K}
\end{array}\right]
$$

with $K=g / B \rho$. A defocusing quadrupole has the transfer matrix

$$
\left[\begin{array}{ll}
\cosh \ell \sqrt{-K} & \frac{1}{\sqrt{-K}} \sinh \ell \sqrt{-K} \\
\sqrt{-K} \sinh 2 \sqrt{-K} & \cosh 2 \sqrt{-K}
\end{array}\right] .
$$

Recall that a quadrupole focusing on the $x$-coordfnate is defocusing on the z-coordinate. A non-focusing section, often called a drift-space, has the transfer matrix appearing in (59),

because in such a section $y^{\prime}$ is constant and $y(s+\Delta s)=y(s)+y^{\prime} \Delta s$.

The transfer matrix for one revolution (91), given earlier, can be obtained as the product of the transfer matrices for the individual segments comprising the ring. In this manner, the Twiss parameters (83)' and the tune value can be determined. 
3.7 Thin Lens Approximation and the FODO Lattice

If we consider a quadrupole in the limit

$$
\ell \rightarrow 0, R+\infty, \ell R+\frac{1}{\mathrm{E}} \text { (finite), }
$$

the transfer matrix (.59); (92) is well-approximated by

$$
\left[\begin{array}{ll}
1 & \ell / 2 \\
0 & 1
\end{array}\right]\left[\begin{array}{ll}
1 & 0 \\
-1 / f & 1
\end{array}\right]\left[\begin{array}{ll}
1 & \ell / 2 \\
0 & 1
\end{array}\right] \text {. }
$$

This is called the thin lens approximation, since the quadrupole of length

$l$ is replaced by a thin lens at its center of focal length $f$, bounded by two drift spaces of length $\ell / 2$. Let us write (93) as. $\underline{D}(\ell / 2) \underline{Q}(f) \underline{D}(\ell / 2)$, defining the matrices $\underline{\underline{D}}(\ell / 2)$ for a drift space of length $\ell / 2$ and $\underline{Q}(f)$ for a thin lens of focal length $f$.

The simplegt alternating gradient magnet arrangement is the FODO lattice, designated by letters to represent focusing, non-focusing, defocusing, non-focusing. By the term "focusing quadrupole" one designates a magnetic

link having horizontally focusing and vertically defocusing properties. One can construct a ring composed of cells, each having the FODO structure. The transfer matrix through one such ceil, Fig. 12, can be written in the thin lens approximation

$$
\underline{M}(f)=\underline{Q}(f / 2) \underline{\underline{D}}(\tilde{L}) \underline{Q}(-f) \underline{D}(\tilde{L}) \underline{Q}(f / 2) \text {. }
$$




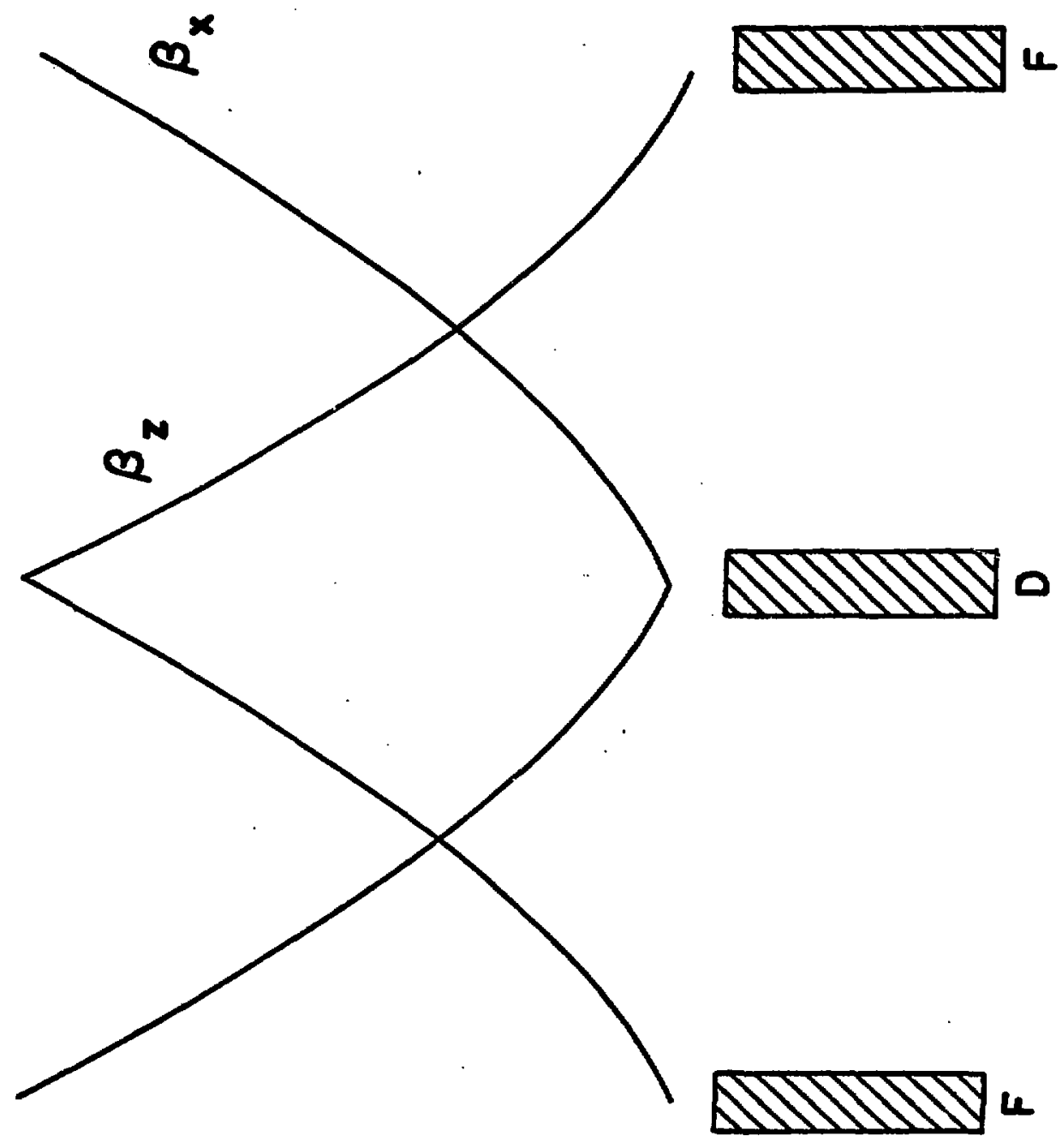


where we start and end in the center of a focusing quadrupole, use $Q(f)=Q(f / 2) Q(f / 2)$, and take the centers of the focusing and defocusing quadrupoies to be a distance $\tilde{L}$ apart. Pexforming the matrix product we obtain

$$
M(f)=\left|\begin{array}{cc}
1-\frac{\tilde{L}^{2}}{2 f^{2}} & 2 \tilde{L}\left(1+\frac{\tilde{L}}{2 f}\right. \\
-\frac{\tilde{L}}{2 f^{2}}\left(1-\frac{\widetilde{L}}{2 f}\right) & 1-\frac{\tilde{L}^{2}}{2 f^{2}}
\end{array}\right| \text {. }
$$

The phase advance $2 \pi v$ through the cell is determined from the trace,

$$
\cos 2 \pi v-\mid=1-\frac{\tilde{L}^{2}}{2 f^{2}},
$$

and the value of the $\beta$-function at the center of the focusing quadrupole is

$$
B_{\max }=\frac{M_{12}}{\cos 2 \pi v}=\frac{2 \tilde{L}(1+\sin \pi v)}{\sin 2 \pi v} i
$$

At the center of the defocusing quadrupole, one has the value $\beta_{\min }$ and

$$
\beta_{\min } / \beta_{\max }=(1-\sin \pi v) /(1+\sin \pi v) \text {, }
$$

which follows immediately since the transfer matrix through a cell beginning In the center of the defocusing quadrupole is $\underline{M}(-f)$.

As shown in Fig. 12 the horizontal and vertical amplitude functions are identical, except that they are out of phase, with $\beta_{x}\left(\beta_{z}\right)$ at maxima (minima) in 
the focusing quadrupoles and at minimum (maximum) in the defocusing quadrupole. This characteristic of alternating gradient focusing provides stability of both the horizontal and vertical betatron oscillations, despite the fact that a single quadrupole is defocusing in one coordinate.

\subsection{Magnetic Field Perturbations}

Magnetic fields in real storage ringseviate from the ideal fields discussed thus far, and treatment of trajectories in such fields requires the addition of perturbation terms to Eqs. (70) The equations of motion become

$$
\begin{aligned}
x^{\prime \prime}+\left[K(s)+G^{2}(s)\right] x & =G(s) \frac{\Delta p}{p}-\frac{\Delta B_{z}(s, x, z)}{B p} \\
z^{\prime \prime}-K(s) z & =\frac{\Delta B_{x}(s, x, z)}{B \rho}
\end{aligned}
$$

and it is convenient to characterize the magnetic field perturbations by harmonic expansions, viz.,

$$
\begin{aligned}
& \Delta B_{z}=b_{0 z}(s)+b_{1}(s) x+a_{1}(s) z+\frac{1}{2} b_{2}(s)\left(x^{2}-z^{2}\right)+a_{2}(s) x z+\cdots \\
& \Delta B_{x}=b_{0 x}(s)+b_{1}(s) z+a_{1}(s) x+b_{2}(s) x z+\frac{1}{2} a_{2}(s)\left(z^{2}-x^{2}\right)+\cdots
\end{aligned}
$$

Dipole field errors $b_{0 z}(s)$ and $b_{0 x}(s)$ are produced, for example,

by errors in the placement of quadrupole magnets, which can result in

deviations of their centers from the reference orbit. Dipole errors 
cause the actual closed orbit, $y_{c o}$, to be displaced from the reference orbit. One can show [see e.g. Sands (1970)] that the displacement in $x$ is given by

$$
x_{c o}(s)=\frac{\beta_{z}^{I / 2}(s)}{2 \sin \pi \nu} \int_{x}^{s+L} d \tilde{s} \beta_{x}^{1 / 2}(\tilde{s}) \frac{b_{p_{2}}(s)}{B \rho} \cos \left[\nu_{x}\left[-\pi+\phi_{x}(s)-\phi_{x}(s)\right]\right],
$$

and a similar relation obtains for $\dot{z}$. The phase $\phi_{x}$ was defined in (78a). We see that the orbit displacement is resonant near integer values of the : tune $v$, which must therefore be avoided. Field errors occurring at locations where the $\beta$-function is large have the greatest influence on the closed

orbit, and the biggest orbit distortions tend to occur at the maxima of the

amplitude function.

Errors in the quadrupole field $b_{1}(s)$, e.g. caused by fluctuations in the power supplies, result in a tune shift [see e.g. Sands (1970)]

$$
\Delta v_{y}= \pm \frac{1}{4 \pi} \int_{0}^{L} d s \beta_{y}(s) \frac{b_{1}(s)}{B \rho}
$$

where $y \quad$ is $x$ or $z$ and the minus sign applles for the z-ccordinate. The tune shift is most sensitive to those gradient errors occurring at locations of maximum amplitude function. One can show [see e.g. Courant and Snyder (1958)] also that the betatron oscillations are driven to instability by gradient errors, if the tune value is near a half-integer.

A skew quadrupole field $a_{1}(s)$ can result from rotation of a quadrupole about its symmetry axis, and such errors introduce coupling between horizontal 
and vertical oscillations. Near the resonance $v_{x}+v_{z}=$ integer, the coupling terms lead to unstable oscillations, while near $v_{x}-v_{z}=$ integer the oscillations remain bounded, though the transfer of oscillation energy between $x$ and $z$ motions becomes large. It is this coupling which limits the extent to which one can succeed in contracting the vertical beam size.

The sextupole field $b_{2}(s)$ and the skew sextupole field $a_{2}(s)$ make the betatron oscillations nonlinear. Resonances to be avoided are $B v_{x}=$ integer, $v_{x} \pm 2 v_{z}=$ integer $]$ and $\left[v_{z}=\right.$ integer, $2 v_{x} \pm v_{z}=$ integer $]$ for the sextupole and skew sextupole flelds, respectively. Nonlinear components such as sextupole and octupole fields arise not only from errors, but are intentionally incorporated as elements of the storage ring. Nonlinearities are essential to the operation of the ring with high electron beam currents. Sextupole magnets are used to correct the dependence of the tune on momentum deviation $\Delta \mathrm{p}$ and thus to avoid the "head-tail" Instability within an electron bunch. Octupoles produce a dependence of tune on oscillation amplitude, which results in Landau damping of instabilities. We emphasize that the choice of tune value is critical to the satisfactory operation of a storage ring in the presence of the inevitable magnetic field 
errors. This is not trivial because $\cdot v_{\mathbf{x}}-v_{z}$ space has many potential resonances as is seen in Fig. 13. It must be kept in mind also that field perturbations existing at locations corresponding to large $\beta$-values have the greatest effect, hence unnecessarily large values of the amplitude functions should be avoided. This is a point to be considered in the design of a low- $\beta$ insertion, a region providing high brightness. It follows from Eq. (80) that in a non-focusing section, 1.e. $(K(s)=0)$,

$$
B(s)=B^{*}+\left(s-s_{0}\right)^{2} / B^{*} \text {. }
$$

where so is the position of the minimum of the amplitude function; usuâty the center of the insertion. The smaller the minimum value $\beta^{*}$, the faster the rise of the $\beta$-function at $s$ values away from $s_{0}$. Hence, very small values of $\beta^{*}$ result in very large values of $\beta$ in the quadrupoles bounding the insertion and thus in great sensitivity to errors in these quadrupoles. An example of a low- $\beta$ insertion is shown in Fig. 14.

3:9 Example: The Lattice of the Brookhaven X-Ray Ring

Eight identical superperiods, one of which is diagrammed in Fig. 14, make up the magnetic lattice of the NSLS 2.5-GeV ring. under construction at Brookhaven. A superperiod is symmetrical about the focusing quadrupole QF, which is located between members of a pair of dipole magnets $B B$. The insertions are bounded at each end by a quadrupole triplet Q1 (defocusing), Q2 (focusing), and Q3 (defocusing); gradients 


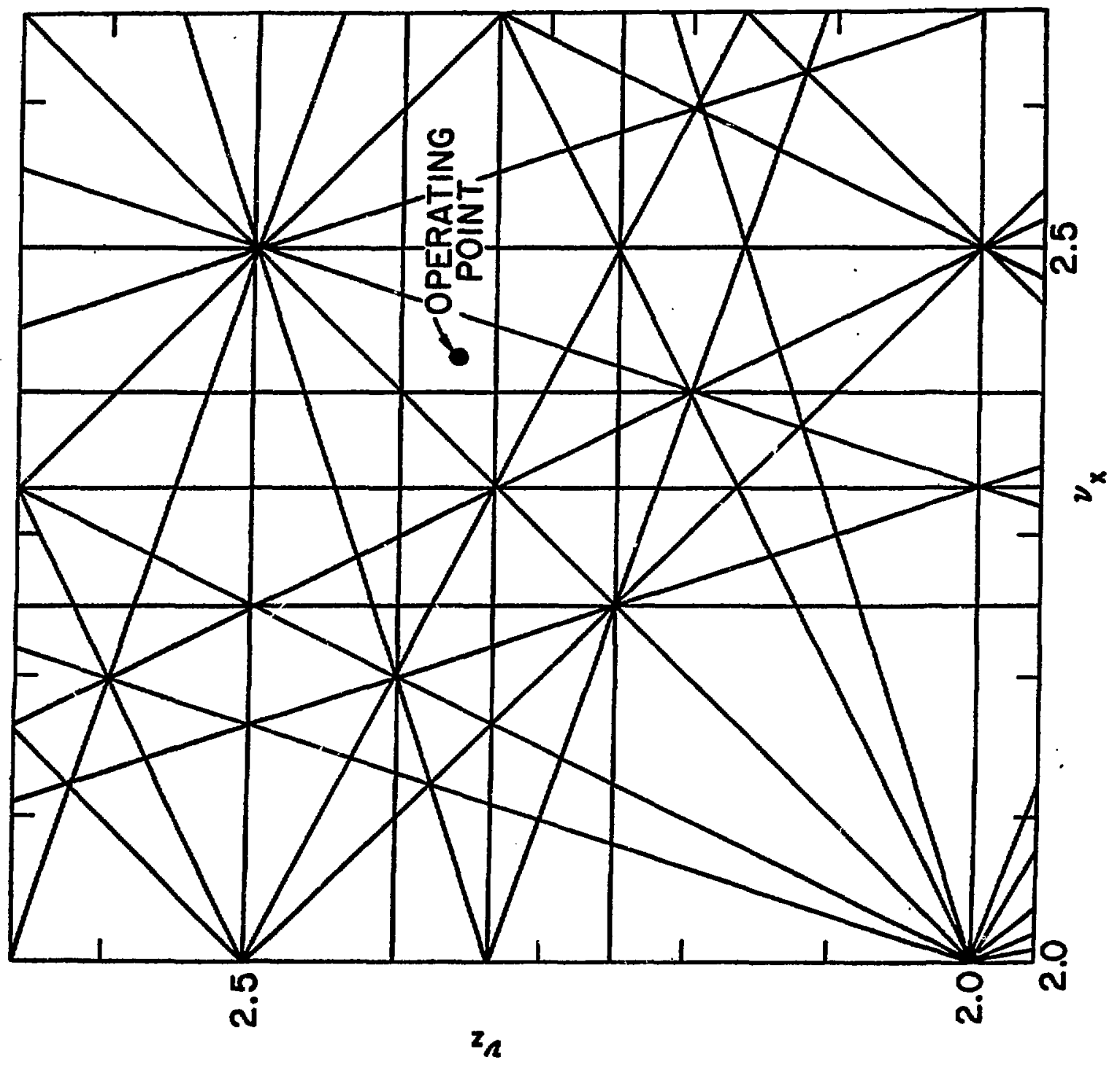




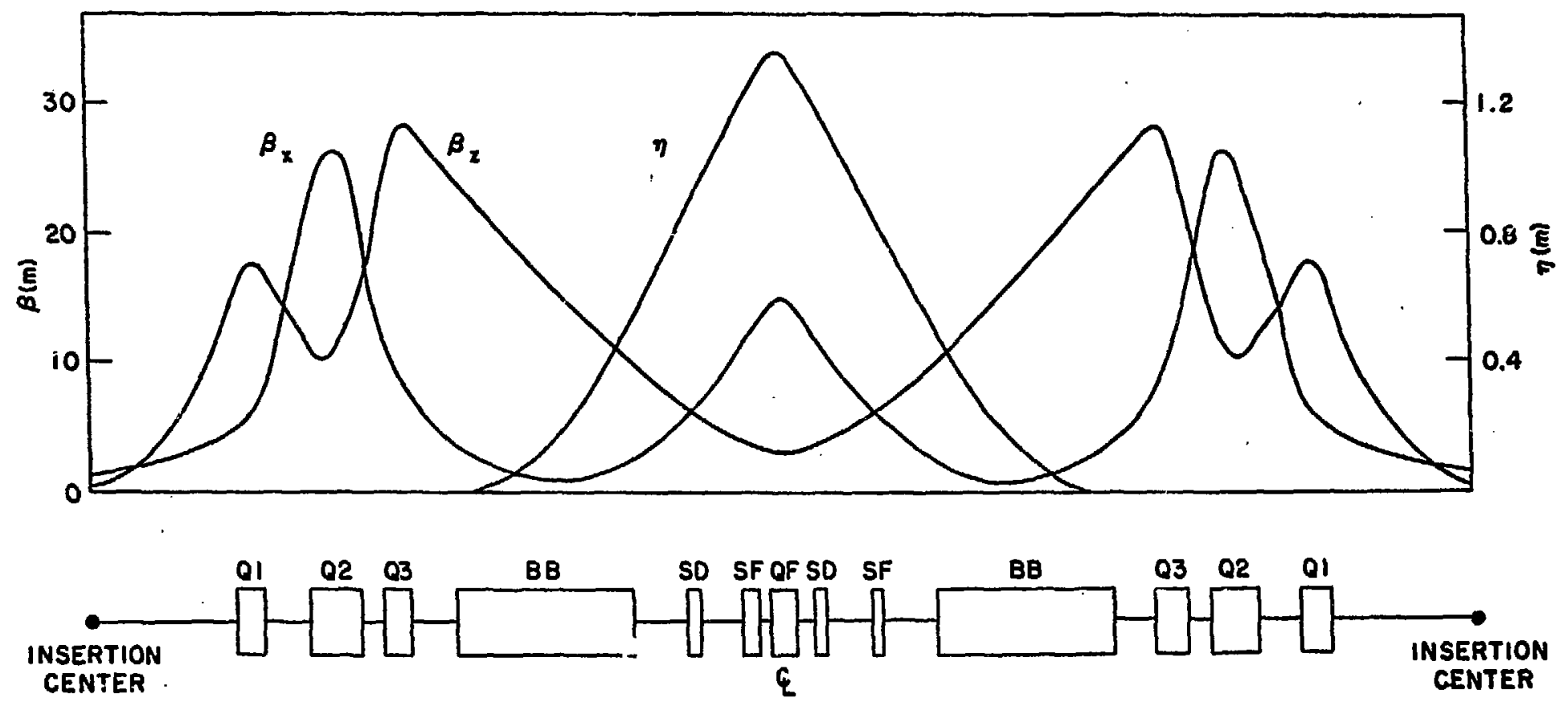


in the triplets are chosen to yield small values of $\beta_{x}$ and $\beta_{z}$ at the insertion center,

IC. The amplitude functions increase from their minimum values at the center

to large values at the triplet, as described by (102).. One notes further that

$\beta_{x}$ and $\beta_{z}$ are out of phase, as expected for an alternating gradient lattice.

SD and SF are sextupole magnets.

In Fig. 14 we also show the horizontal dispersion $n$, which is localized

between the two dipole magnets and fisero in the insertion. This results

from the choice of gradient in QF. Recall from Eq. (72) that

$$
\eta^{\prime \prime}+\left[K(s)+G^{2}(s)\right] \eta=G(s),
$$

so, if $\eta=\eta^{\prime}=0$ at the IC., it remains zero until a region of non-zero

curvature is reached. The dipole bending magnet field acts as a driving term

increasing the dispersion to positive values, and $n$ continues to increase

until it is focused by $Q F$. In $Q F$ the gradient is chosen such that $\eta^{\prime}=0$ at

its center. Such a combination, two dipoles with a quadrupole between

them having the proper gradient to localize the dispersion, is called an

"achromatic bend". 


\subsection{Synchrotron Oscillations, Radiation Daming}

The energ. an electron loses as synchrotron radiation must be restored by one or more RF cavities located around the orbit. Thus, a time varying . field effects the energy transfer and, for one RF cavity whose field has a sinusoidal time dependence, the energy gained by an electron arriving at time $t$ can be written

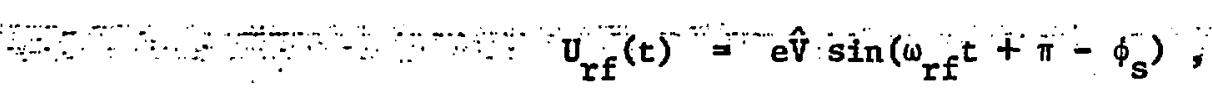

where $\hat{v}$ is the peak $R F$ voltage, $\omega_{r f}$ the $R F$ frequency, and $\phi_{S}$ is a phase difference Gpecified below and Indicated in Fig. 15) between the electron motion and the cavity field.

For a given current excitation of the storage ring magnets, one has in mind an ideal orbit corresponding to electron energy $E_{0}$. The circumference of this orbit is $L$ and the period is $T_{0}=L / c$. One chooses the RF frequency to be an integral multiple of the revolution frequency,

$$
\omega_{\mathrm{rf}}=2 \pi \mathrm{h} / \mathrm{T}_{0} ;
$$

the integer $h$ is called the harmonic number. The energy lost to synchrotron radiation by an electron on the ideal orbit is $v_{0}$, and for such an electron in the

"synchronous phase" this energy loss is fust made up by/cavity field at $t=0$; 


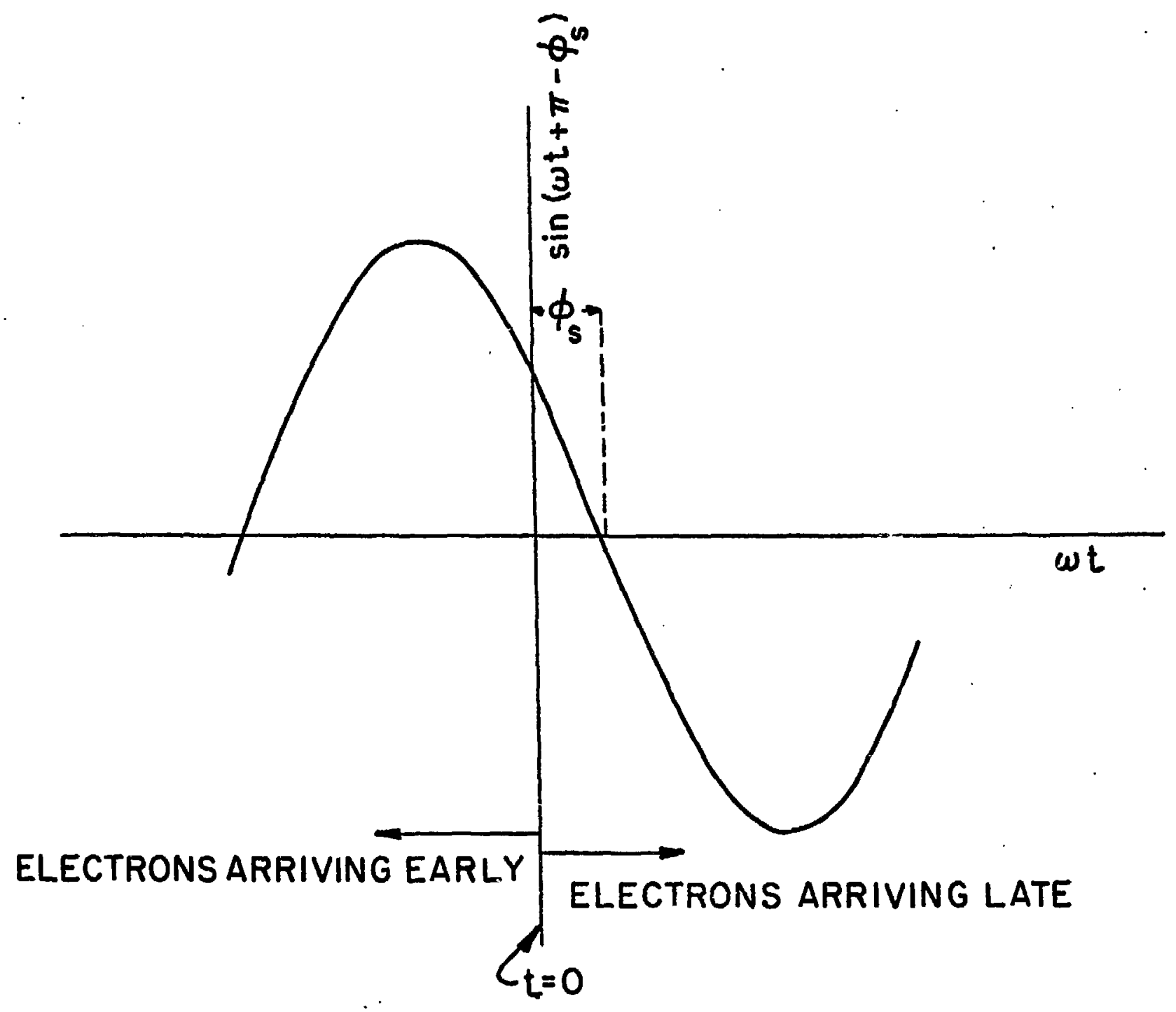


that is,

$$
\begin{aligned}
& u_{0}=u_{r f}(t=0)=e^{\hat{V}} \sin \left(\pi-\phi_{S}\right)=e \hat{V} \sin \phi_{S} \\
& \left(0 \leq \phi_{S} \leq \pi / 2\right)
\end{aligned}
$$

Such a "synchronous electron" will then remain at energy $E_{0}$ on the ideal

orbit, and its arrival time at the cavity satisfies the relation

$$
t_{s}=\text { integer } \times T_{0} / h
$$

There are potentialiy h equaliy space synchronous

electrons moving around the ring, and each can be the center of a bunch of electrons undergoing synchrotron oscillations about It.

To derive the equations describing synchrotron oscillations, we consider

an electron of energy $E_{0}+\varepsilon$ which arrives at the $R F$ cavity a time interval

T ahead of a synchronous electron. The closed orbit corresponding to energy

$E_{0}+\varepsilon$ has a larger circumference $L_{\varepsilon}$ than the ideal orbit,

$$
\left(L_{\varepsilon}-L\right) / L=\alpha \varepsilon / E_{0}
$$

where $\alpha$ is the momentum compaction [Eqs. (75) and (76)]. The corresponding relation for the orbit period is

$$
\left[T(\varepsilon)-T_{0}\right] / T_{0}=\alpha \varepsilon / E_{0}
$$

After one revolution the time interval $\tau$ will have been.reduced 
by $T(\varepsilon)-T_{0} ;$ thus

$$
\frac{d \tau}{d t}=-\alpha \varepsilon / E_{0}
$$

The energy lost per turn to synchrotron radiation by the electron of energy

$E_{0}+\varepsilon$ is $U_{\text {rad }}(\varepsilon)$, with $U_{\text {rad }}(0)=U_{0}$; the energy gained at the RF cavity is

$$
U_{r f}\left(t_{s}-\tau\right) \equiv \operatorname{eV}(\tau)=e^{\hat{V}} \sin \left(\omega_{r f} \tau+\phi_{s}\right)
$$

After one revolution the energy deviation $\varepsilon$ will be increased by eV $(\tau)$ -

$$
\mathrm{U}_{\mathrm{rad}}(\varepsilon) \text { so }
$$

$$
\frac{d \varepsilon}{d t}=\frac{e V(\tau)-u_{r a d}(\varepsilon)}{T_{0}} .
$$

Equations (109) and (111) provide a description of the synchrotron oscillations. Suppose $\tau$ and $\varepsilon$ are sufficiently small so that these equations can be

Iinearized,

$$
e V(\tau) \cong U_{0}+\left[e \hat{V}_{r f} \cos \phi_{s}\right] \tau,
$$

and

$$
\mathrm{U}_{\mathrm{rad}}(\varepsilon) \cong \mathrm{U}_{0}+\left[\frac{\mathrm{dU}}{\mathrm{rad}}\right]_{\delta=0} \varepsilon
$$

Equations (109) and (111) can then be combined to obtain

$$
\frac{d^{2} \tau}{d t^{2}}+2 \alpha_{\varepsilon} \frac{d \tau}{d t}+\Omega^{2} \tau=0
$$

with 


$$
\begin{gathered}
\alpha_{\varepsilon}=\frac{1}{2 T_{0}}\left[\frac{\mathrm{dU}}{\mathrm{rad}}\right]_{\varepsilon=0}, \\
\Omega_{s}^{2}=\frac{e \hat{V} \alpha \omega_{r f} \cos \phi_{s}}{E_{0} T_{0}}=\frac{2 \pi e h \hat{v} \alpha \cos \phi_{s}}{E_{0} T_{0}^{2}}
\end{gathered}
$$

Equation (112) represents a damped harmonic oscillation having angular

syachrotron oscillation frequency $\Omega_{s}$ and damping coefficient $a_{\varepsilon}$. The oscillations are stable $\left(\Omega_{s}^{2}>0\right)$ for $\cos \phi_{s}>0$ and unstable for $\cos \phi_{s}<0$.

This behavior led ' us, in Eq. (105), to restrict attention to $0 \leq \phi_{s} \leq \pi / 2$.

$$
\text { To evaluate the damping constant } \alpha_{\varepsilon} \text {, we compute the derivative }
$$

$\left[\mathrm{dU} \mathrm{rad}_{\mathrm{rad}} / \mathrm{d} \varepsilon\right]_{\varepsilon=0^{*}}$ With $\mathrm{P}$ as the power radiated by an electron of energy $\mathrm{E}$

in a magnetic field $B$, the energy loss is

$$
v_{\text {rad }}=\oint \frac{d \ell}{c} P .
$$

If ds is the arc length projection on the ideal orbit which corresponds to

a distance $d l$ travelled by an electron of energy $E_{0}+\varepsilon$ in a region where

the inverse radius of curvature is $G$ and dispersion is $\eta$, we may write

[Eq. (65)]

$$
d l=\left(I+G n \frac{\varepsilon}{E_{0}}\right) d s
$$

We find

$$
u_{\text {rad }}(\varepsilon)=\oint \frac{d s}{c}\left[1+\eta(s) G(s) \frac{\varepsilon}{E_{0}}\right] P(s)
$$


and hence

$$
\left[\frac{\mathrm{dU}}{\mathrm{rad}}\right]_{\varepsilon=0}=\oint \frac{\mathrm{ds}}{\mathrm{C}}\left[2 \frac{\mathrm{P}}{\mathrm{E}_{0}^{-}}+2 \frac{\mathrm{P}}{\mathrm{B}} \frac{\mathrm{dB}}{\mathrm{dE}}+\eta \mathrm{G} \frac{\mathrm{P}}{\mathrm{E}_{\mathrm{O}}}\right] \text {. }
$$

Since

$$
\frac{1}{B} \frac{d B}{d E}=\frac{1}{B} \frac{d B}{d x} \frac{d x}{d E}=\frac{K}{G} \frac{n}{E_{0}}
$$

and

$$
U_{0}=\oint \frac{d s}{c} P=T_{0}\langle P\rangle
$$

where $T_{0}$ is the revolution period : and $\langle P\rangle$ is the average power. it

follows from Eqs. (113) and (117) that

$$
{ }_{\varepsilon}=\frac{U_{0}}{2 \mathrm{E}_{0} T_{0}}(2+\infty),
$$

with

$$
\mathscr{D}=\frac{\oint d s G(s) n(s)\left[2 R(s)+G^{2}(s)\right]}{\oint d s G^{2}(s)} .
$$

Let us evaluate $\mathcal{D}$ in two cases: (1) an isomagnetic separated function

lattice, (2) an isomagnetic combined function lattice. By isomagnetic we mean that where the dipole field is nonzero, it always has the same value, i.e.

$$
G(s)= \begin{cases}G_{0} & \text { in dipoles, } \\ 0 & \text { elsewhere. }\end{cases}
$$

Separated function means that the gradient is zero where the dipole field is non-zero, and visa-versa, 1.e. 


$$
G(s) K(s)=0 .
$$

For the isomagnetic, separated function lattice, Eq. (121) reduces to

$$
\Phi=\frac{G_{0}^{2}}{2 \pi} \int_{M a g} n(s) d s \text {, }
$$

where the integral of the dispersion function extends only ovex the dipole

magnets. The momentum compaction can be written in terms of the dispersion

function as [Eq. (76)]

$$
\alpha=\frac{1}{L} \oint d s n(s) G(s) .
$$

Here, as in the foregoing, L.designates the total length or perimeter of the orbit. With $L=2 \pi R$, $R$ being the effective radius of the ring,

$$
\alpha=\frac{G_{0}}{2 \pi R} \int_{M a g} n(s) d s ;
$$

and then

$$
\Phi=\frac{R \alpha}{\rho_{0}}, \text { (isomagnetic, separated function) }
$$

where $\rho_{0}$ is the dipole bending radius.

In the combined function lattice all magnets have an inverse radius of curvature $G(s)=G_{0}$, and the focusing gradient $K$ is non-zero in at least some of them. Since the dispersion function satisfies [Eq. (72)] 


$$
n^{\prime \prime}+\left[K(s)+G^{2}(s)\right] n=G(s)
$$

with

$$
n(s+L)=n(s) \text {, }
$$

one obtains

$$
\oint d s G n\left(R+G^{2}\right)=G_{0} \oint d s n\left(R+G^{2}\right)=2 \pi G_{0} \text {. }
$$

It then follows from (121) that

$$
\mathcal{D}=2-\frac{R \alpha}{\rho} \text { (combined function). }
$$

The implications of these two values for $\mathscr{W}$ and hence for $\alpha_{\varepsilon}$ are considered below.

\subsection{Robinson's Theorem}

Radiation damping of the transverse betatron motion is most easily treated using the powerful theorem derived by Robinson (1958): We consider the $6 \times 6$ transfer matrix for going from position $s_{1}$ to $s_{2}$

$$
\left[\begin{array}{l}
x \\
x^{\prime} \\
z^{\prime} \\
\tau \\
\varepsilon / E_{0}
\end{array}\right]_{2}=\left[M\left(s_{2} / s_{1}\right) \mid\left[\begin{array}{l}
x \\
x^{\prime} \\
z \\
z^{\prime} \\
\tau \\
\varepsilon / E_{0}
\end{array}\right]_{1} \cdot\right.
$$

For one complete revolution $\left(s_{2}=s_{1}+L\right)$, the elgenvalues of the matrix

$\underline{M}\left(s+L_{1} / s\right)$ are $\exp \left(-\tilde{\alpha}_{j} \pm 1 \tilde{\beta}_{j}\right), j=1,2,3$; and the determinant is 


$$
\operatorname{det} \underline{M}(s+L / s)=\exp \left(-\sum_{j=1}^{3} 2 \tilde{\alpha}_{j}\right) \cong 1-\sum_{j=1}^{3} 2 \tilde{a}_{j} \cdot
$$

The amplitudes of the three oscillation modes behave like $\exp \left(-\alpha_{j} t\right)$, where

$$
\alpha_{j}=\tilde{\alpha}_{j} / T_{0},
$$

and $T_{0}$ is the revolution period.

For transfer from $s$ to $s+d s$ the matrix can be written $\underline{M}(s+\mathrm{ds} / s)=\underline{1}+\underline{\underline{m}} \mathrm{ds}$, and its determinant is

$$
\operatorname{det} \underline{M}(s+d s / s) \cong 1+(\operatorname{Tr} \underline{M}) d s ;
$$

(Try) is the trace of the matrix, that is, only diagonal terms contribute.

Diagonal terms for $x, z$, or $\tau$ are nonexistent, since e.g. $x+x+x^{\prime} d s$,

$z \rightarrow z+z^{\prime} d s$, and $\tau \rightarrow \tau+f(\varepsilon) d s$. On the other hand, there are diagonal terms for $x^{\prime}, z^{\prime}$, and $\varepsilon$. Those for $x^{\prime}$ and $z^{\prime}$ arise from the energy gain

$\delta \varepsilon_{1}$ in traversing an element ds of an RF cavity. As one sees from Fig. 16; the momentum galned by the electron is in the longitudinal direction and

$$
\delta x^{\prime}=-\left(\delta \varepsilon_{1} / E_{0}\right) x^{\prime}
$$

The point is that the change in angle is proportional to the incident angle, hence one has the diagonal terms

$$
x^{\prime} \rightarrow\left(1-\frac{\delta \varepsilon_{1}}{\varepsilon_{0}}\right) x^{\prime},
$$

and

$$
z^{\prime} \rightarrow\left(1-\frac{\delta \varepsilon_{1}}{E_{0}} z^{\prime}\right.
$$




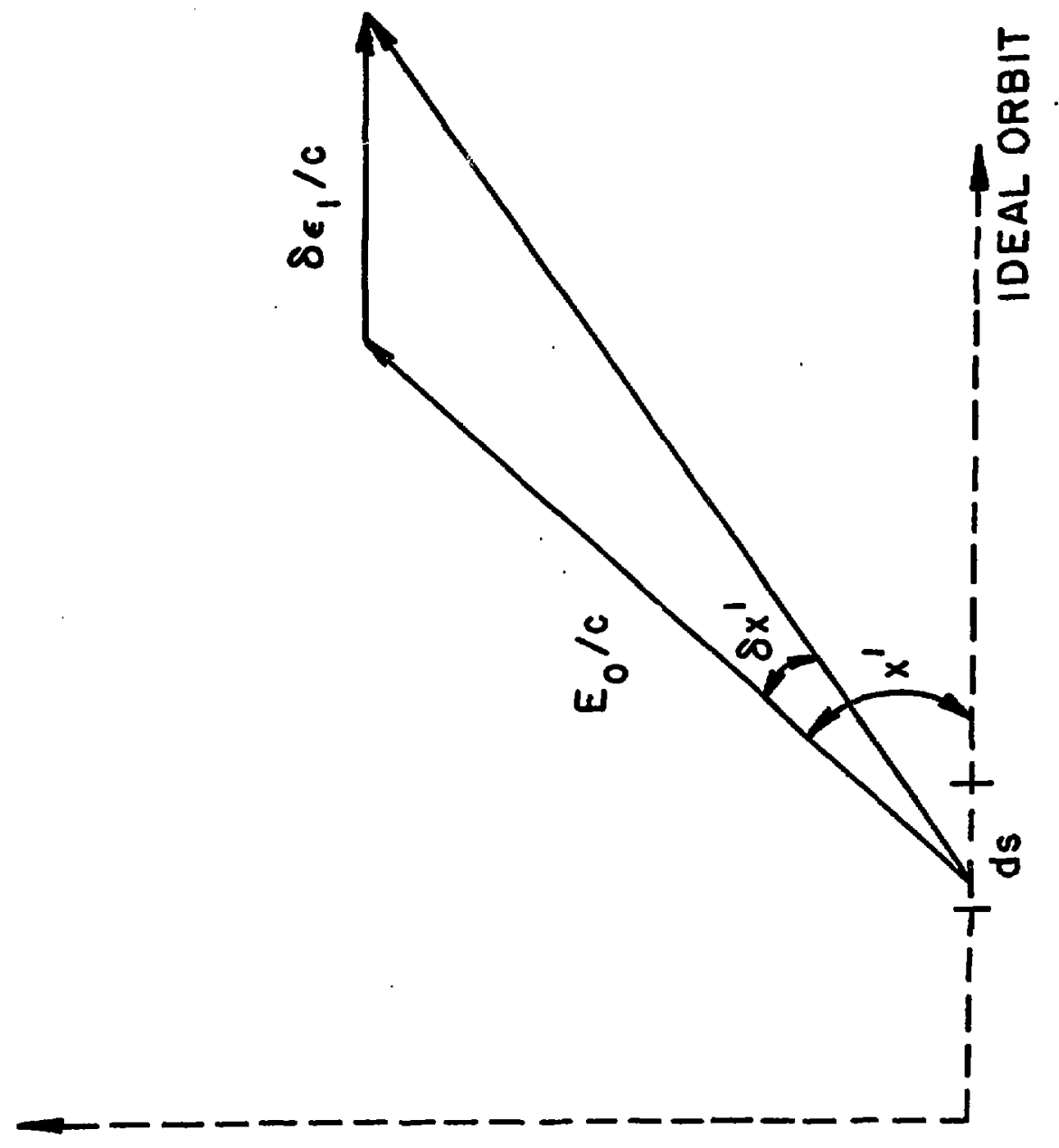


It follows from Eq. (35c) or (35e) that the energy lost to synchrotron

radiation in the interval $s \rightarrow s+d s$ by an electron of energy $E_{0}+\varepsilon$ is

$$
\delta U(\varepsilon)=\left(1+2 \varepsilon / E_{0}\right) \delta \varepsilon_{2},
$$

where $\delta \varepsilon_{2}$ is the energy lost by an electron of energy $E_{0^{*}}$ This results in

the diagonal term

$$
\varepsilon \rightarrow\left[1-2 \delta \varepsilon_{2} / E_{0}\right] \varepsilon
$$

Using Eq. (126) together with $(128 a, b)$ and $(130)$, we find

$$
\operatorname{det} \underline{M}(s+d s / s)=1-2 \frac{\delta \varepsilon_{1}}{E_{0}}-2 \frac{\delta \varepsilon_{2}}{E_{0}}
$$

and

$$
\begin{aligned}
\operatorname{det} \underline{M}(s+L / s) & =\pi \operatorname{det} \underline{M}(s+d s / s) \\
& =1-2 \frac{\varepsilon_{1}^{0}}{E_{0}}-2 \frac{\varepsilon_{2}^{0}}{E_{0}},
\end{aligned}
$$

where $\varepsilon_{1}^{0}$ is the energy gain from the RF in one revolution and $\varepsilon_{2}^{0}$ is the energy lost to synchrotron radiation in one revolution. In equilibrium obviously, $\varepsilon_{1}^{0} \cdot=\varepsilon_{2}^{0}=U_{0}$. Combining Eq. (132) with (124) and (125), one obtains

$$
\sum_{j=1}^{3} \alpha_{j}=\frac{2 U_{0}}{E_{0} T_{0}}
$$

the relation known as Robinson's theorem. 
In the absence of coupling between the $x$ and $z$ betatron oscillations,

the transfer matrix is block diagonal with a $2 \times 2$ z-z' block and a $4 \times 4$ $x-x^{\prime}-\tau-\varepsilon$, block. If the argument leading to (133) is applied separately

to each block, we obtain

$$
a_{x}+a_{\varepsilon}=\frac{3 U_{0}}{2 E_{0} T_{o}^{2}}
$$

and

$$
a_{z}=\frac{U_{0}}{2 E_{0} T_{0}} \equiv \tau_{z}^{-1}
$$

From Eq. (120) we know that

$$
a_{\varepsilon}=(2+\infty) \frac{U_{0}}{2 E_{0} T_{j}} \equiv \tau_{\varepsilon}^{-1}
$$

hence

$$
a_{x}=(1-D) \frac{U_{0}}{2 E_{0} T_{0}} \equiv \tau_{x}^{-1}
$$

with $\theta$ defined in Eq. (121).

In an isomagnetic separated-function lattice, for which $\mathcal{D}$ is given In (122), we have

$$
\alpha_{x}=\left(1-\frac{R a}{\rho_{0}}\right) \frac{U_{0}}{2 E_{0} T_{0}}
$$

Typically $\mathrm{Ra} / \rho_{0}$ is less than unity so that $\alpha_{\mathbf{x}}>0$ and the radial oscillations are damped. In the case of a combined function lattice, however, the situation is different. Here $D_{\text {is given by (123); then }}$

$$
\alpha_{x}=\left(\frac{R a}{\rho_{0}}-1\right) \frac{U_{0}}{2 E_{0} T_{0}}
$$


and, since $\mathrm{Ra} / \rho_{0}$ is less than unity, the radial betatron oscillations are antidamped.

In the no Ionger existing Cambridge Electron Accelerator, which had a combined function lattice, Robinson (1958) suggested that the radial antidamping might be counteracted by installation of a wiggler magnet. His idea was to utilize a magnet intended to reduce the damping rate of energy oscillations

and thereby, according to Eq. (134), increase damping of the radial motion

A wiggler magnet functions in this way if the higher energy electrons passing

through it radiate less than the lower energy electrons. Now, from Eq. (35c)

we know that

$$
P \approx P_{0}\left(1+2 \frac{\Delta E}{E}+2 \frac{\Delta B}{B}\right) \text {, }
$$

and taking

$$
\frac{\Delta B}{B}=\frac{1}{B_{w}} \frac{\partial B_{w}}{\partial x} \eta_{w} \frac{\Delta E}{E}
$$

where $B_{w}$ is the wiggler dipole field, $\partial B_{w} / \partial x$ its gradient, and $\eta_{w}$ is the dispersion in the wiggler, we see that higher energy electrons will indeed radiate less if

$$
\frac{1}{B_{w}} \frac{\partial B_{w}}{\partial x} n_{w} \ll \quad-I .
$$


In summary, the damping rates are given by

$$
\begin{aligned}
& \tau_{j}^{-1}=\alpha_{j}=J_{j} \tau_{0}^{-1}, \\
& \tau_{0}^{-1}=\frac{\langle P\rangle}{2 E_{0}}=U_{0} /\left(2 E_{0} T_{0}\right),
\end{aligned}
$$

where the damplng partition numbers $J_{f}$, which satisfy the relation $J_{1}+J_{2}+J_{3}=$ 4, describe the division of the damping among the three modes. In the absence of $x-z$ coupling,

$$
\mathrm{J}_{\mathrm{x}}=1-\Phi, \mathrm{J}_{2}=1, \mathrm{~J}_{\varepsilon}=2+3,
$$

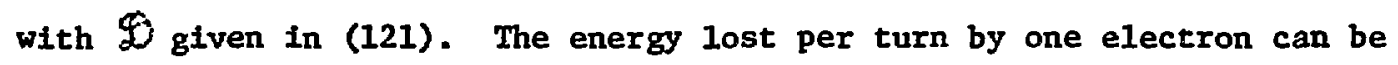
written [Eq. 4a]

$$
U_{0}=\frac{88.5 \mathrm{E}_{\mathrm{o}}^{4}(\mathrm{GeV})}{\rho(\mathrm{m})}(\mathrm{keV} / \text { electron-turn })
$$

for an isomagnetic lattice. If we take the bending radius to be $\rho=10 \mathrm{~m}$, the circumference $L=200 \mathrm{~m}$, and the revolution period $T_{o}=L / c=0.66 \mu \mathrm{sec}$, then for $E_{0}=1 \mathrm{GeV}$, we see that

$$
\begin{gathered}
U_{0}=8.9 \mathrm{keV} / \mathrm{el},-\operatorname{turn} \text { and } \tau_{0}=0.15 \mathrm{sec} \text {; and for } E_{0}=3 \mathrm{GeV}, \\
U_{0}=717 \mathrm{keV} / \mathrm{el} .-\mathrm{turn} \text { and } \tau_{0}=5.5 \mathrm{msec} .
\end{gathered}
$$




\subsection{Quantum Fluctuations}

The power $\mathbf{P}$ emitted as synchrotron radiation by an electron of energy $\mathbf{E}$

In a magnetic field B is given in Eqs. (35) and (4)... If we let $P(\omega)$ d $w$

be the power radiated in the photon frequency interval between $\omega$ and

$\omega+d \omega$, then

$$
P=\int_{0}^{\infty} d \omega Q(\omega)
$$

with

$$
\theta(\omega)=\frac{P}{\omega_{c}} s\left(\frac{\omega}{\omega_{c}}\right) \text {, }
$$

where the characteristic frequency $\omega_{c}$ is given by [Eq. (8)].

Taking into account the fact that the radiation is emitted in discrete photons of energy $u=h w$, we let $n(u) d u$ be the number of photons emitted per sec with energy between $u$ and $u+d u$. Then

$$
u n(u) d u=P\left(\frac{u}{h}\right) \frac{d u}{\hbar} \text {. }
$$

and we may write

$$
n(u)=\frac{9 \sqrt{3} P}{8 \pi u_{c}^{2}} \int_{u / u_{c}}^{\infty} K_{5 / 3}(x) d x,
$$

where the characteristic energy, in $\mathrm{kev}$, is

$$
u_{c}=\hbar \omega_{c}=2.22 \mathrm{E}^{3}(\mathrm{GeV}) / \rho(\mathrm{m})
$$

It may be noted thai: $\mathrm{Eq.} \mathrm{(142)} \mathrm{corresponds} \mathrm{to} \mathrm{(54).}$ 
The number of photons emitted per sec is

$$
N=\int_{0}^{\infty} n(u) d u=\frac{15 \sqrt{3}}{8} \frac{P}{u_{c}} \text {, }
$$

and the average quantum energy is

$$
\langle u\rangle=\frac{1}{N} \int_{0}^{\infty} u n(u) d u=\frac{8}{15 \sqrt{3}} u_{c} \simeq 0.32 u_{c}
$$

The average number of photons emitted by an electron per radian of arc is

$$
\frac{\mathrm{U}_{0}}{2 \pi<u>} \cong 20 \mathrm{E}(\mathrm{GeV})
$$

Since this is a small number, the effects of fluctuations in the rate of photon emission are important.

We have seen that the average power loss to synchrotron radiation damps the energy oscillation in a storage ring. Deviations from the average rate of energy loss result in an energy spread $\sigma_{\varepsilon}$ among the electrons in the beam, since at a given time different electrons will have radiated different amounts of energy. As noted by Sands (1970) the typical energy fluctuation is expected to arise from the deviation from its mean of the number of photons emitted in one damping period ${ }^{\prime} \varepsilon$. If photon emission is assumed to be a Poisson process, i.e. each event is independent of all others, the standard deviation from the mean is 


$$
\sigma_{\varepsilon} \sim \sqrt{\mathrm{N} \tau_{\varepsilon}} u_{c}
$$

and since $\mathrm{N} \sim \mathrm{P} / \mathrm{u}_{\mathrm{c}}$ and $\tau_{E} \sim \mathrm{E}_{\mathrm{o}} / \mathrm{P}$,

$$
c_{\varepsilon} \sim \sqrt{E_{o} u_{c}} \text {, }
$$

the geometric mean of the electron energy and the tripical photon energy.

This shows that the energy spread in the electron beam is very much larger

than the photon energy. For a 1-GeV electron and $p=10 \mathrm{~m}, u_{c}=0.22 \mathrm{keV}$ and $\sigma_{\varepsilon} \sim 0.5 \mathrm{MeV}$

\subsection{Equilibrium Energy Distribution and Bunch Length}

In the absence of quantum fluctuations, the synchrotron oscillations are described by Eqs. (109) and (111). To include the effect of quantum fluctuations we replace the linearized version of Eq. (111) by

$$
\frac{d \varepsilon}{d t}=\frac{e V_{1} \tau-\nabla_{1} \varepsilon}{T_{0}}-[\tilde{P}(t)-P],
$$

where

$$
\begin{aligned}
& \tilde{P}(t)=\Sigma_{i} u_{i} \delta\left(t-t_{i}\right), \\
& v_{1}=\hat{v}_{\omega_{\tau f}} \cos \phi_{s},
\end{aligned}
$$

and

$$
\mathrm{U}_{1}=\left[\frac{\mathrm{d} U_{\mathrm{rad}}}{\mathrm{d} \varepsilon}\right]_{\varepsilon=0}
$$

This is a stochastic equation in which $u_{i}$ and $t_{i}$ iie random variables. From (149) together with (109) one obtains 


$$
\frac{d^{2} \varepsilon}{d t^{2}}+2 \alpha_{\varepsilon} \frac{d \varepsilon}{d t}+\Omega_{s}{ }^{2} \varepsilon=-\frac{d}{d t}[\tilde{P}(t)-P] .
$$

with $\alpha_{\varepsilon}=\mathrm{U}_{1} / 2 \mathrm{~T}_{0}$ and $\Omega_{s}^{2}=\mathrm{eV}_{1} \alpha / \mathrm{E}_{0} \mathrm{~T}_{0}$. It follows that

$$
\varepsilon(t)=\frac{-1}{\Omega_{s}} \int_{-\infty}^{t} d t^{\prime} \frac{d P\left(t^{\prime}\right)}{d t^{\prime}} e^{-\alpha_{\varepsilon}\left(t-t^{\prime}\right)} \sin \Omega_{s}\left(t-t^{\prime}\right) .
$$

When $\alpha_{\varepsilon} \ll \Omega_{s}$, integration by parts together with Eq. (150) ylelds

$$
-\varepsilon(t)=-\sum_{t_{i}=-\infty}^{t} u_{i} e^{-a_{\varepsilon}\left(t-t_{i}\right)} \cos \Omega_{B}\left(t-t_{i}\right) \cdots
$$

Since values of $E(t)$ are normally much larger than typical photon

energies and since the $t_{1}$ are randomly distributed, the $\varepsilon(t)$ sum consists of

a large number of statistically independent, thus randomly positive and

negative, small terms. The Central Limit Theorem implies that the probability

that the energy $\varepsilon(t)$ is between $\varepsilon$ and $\varepsilon+d \varepsilon$ is

$$
w(\varepsilon) d \varepsilon=\frac{1}{\sqrt{2 \pi} \sigma_{\varepsilon}} e^{-\varepsilon^{2} / 2 \sigma_{\varepsilon}^{2}} \mathrm{~d} \varepsilon .
$$

To estimate $\sigma_{\varepsilon}$ let us consider the quantity

$$
A_{s}^{2}=\varepsilon^{2}+\frac{1}{\Omega_{s}{ }^{2}}\left(\frac{d \varepsilon}{d t}\right)^{2},
$$

which would be invariant in the absen'e of radiation damping and quantum 
fluctuations. The emission of a photon of energy u results in the change

or

$$
A_{s}^{2}+\delta A_{s}^{2}=(\varepsilon-u)^{2}+\frac{1}{\dot{\Omega}_{s}^{2}}\left(\frac{d \varepsilon}{d t}\right)^{2}
$$

$$
\delta A_{s}^{2}=-2 \varepsilon u+u^{2} \text {. }
$$

Since $\varepsilon$ is as likely to be positive as negative,

$$
\left\langle\delta A_{s}^{2}\right\rangle=\left\langle u^{2}\right\rangle
$$

this average is over the photon energy distribution (142),

$$
N\left\langle u^{2}\right\rangle=\int_{0}^{\infty} u^{2} n(u) d u=\frac{55}{24 \sqrt{3}} u_{c} P,
$$

with $\mathrm{N}$ being the number of photons emitted per sec given in Eq. (144).

Using (157) together with the change in $A_{s}^{2}$ due to radiation damping, we find

$$
\frac{d<A_{s}{ }^{2}>}{d t}=N<u^{2}>-\frac{2<A_{s}^{2}>}{\tau_{E}} .
$$

At equilibrium $\frac{d_{i}<A_{a}>^{2}}{d t}=0$, hence

$$
\sigma_{E}^{2}=\frac{1}{2}\left\langle A_{s}^{2}\right\rangle=\frac{1}{4} \tau_{E}{ }^{N}\left\langle u^{2}\right\rangle
$$

The bunch length may be obtained from equipartition, by not:ling that the standard deviation of $\frac{1}{\Omega_{s}} \frac{d \varepsilon}{d t}$ should be equal to $\sigma_{\varepsilon^{*}}$ Using Eqs. (109) and (112), we see that for $\alpha_{E} \ll \Omega_{s}$, 


$$
-\Omega_{s}{ }^{2} \tau=\frac{d^{2} \tau}{d t^{2}}=-\frac{\alpha}{E_{0}} \frac{d \varepsilon}{d t},
$$

hence

$$
\frac{1}{\Omega_{s}} \frac{d E}{d t}=\frac{\Omega_{s} E_{0}}{\alpha} \tau .
$$

Equipartition implies that the bunch length, in time,

$$
\sigma_{\tau}=\left(\begin{array}{l}
a \\
\sigma_{s}
\end{array}\right)\left(\begin{array}{l}
\sigma_{s} \\
E_{0}
\end{array}\right)
$$

and the bunch length, in space, $\sigma_{\mathrm{L}}$ 1s just co $\sigma_{\tau}$

To be more precise, we must average $\mathrm{N}\left\langle\mathrm{u}^{2}>\right.$ over the orbit:

$$
\sigma_{\varepsilon}^{2}=\frac{1}{4} \tau \varepsilon \frac{1}{2 \pi R} \oint N<u^{2}>d s .
$$

From (158), (164), and the relations

$$
\begin{aligned}
P & =\frac{2 \pi R<P>G^{2}(s)}{\oint_{G}^{2}(\tilde{s})}, \\
u_{c} & =\frac{3}{2} \hbar c \gamma_{0}^{3} G(s), \\
\tau_{\varepsilon} & =\frac{2 E_{c}}{J_{\varepsilon}<P>},
\end{aligned}
$$

where $J_{E}$ is a damping partition number (138), one finds

$$
\left[\frac{\sigma_{\varepsilon}}{E_{0}}\right]^{2}=\frac{c_{q} \gamma_{0}^{2}}{J_{\varepsilon}} \frac{d s\left|G^{3}\right|}{d s G^{2}}
$$

with

$$
C_{q}=\frac{55}{32 \sqrt{3}} \frac{\hbar}{\mathrm{mc}}=3.84 \times 10^{-13} \mathrm{~m} \text {. }
$$


In the :ase of an isomagnetic lattice, $G(s)=1 / \rho_{0}$ or 0 , hence

$$
\left(\frac{\sigma_{\varepsilon}}{E_{n}}\right)^{2}=\frac{c_{q} r_{0}^{2}}{J_{\varepsilon} \rho_{0}}
$$

With a typical set of parameter values: $\rho_{0}=10 \mathrm{~m}, \mathrm{~J}_{\varepsilon}=2$,

$\Omega_{s}=10^{5} \mathrm{sec}^{-1}, \alpha=10^{-2}$, and for $E_{0}=1 \mathrm{GeV}$ we see that

$\sigma_{\varepsilon} / E_{0}=2.7 \times 10^{-4}$ and $\sigma_{\tau}=2.7 \times 10^{-11} \mathrm{sec} ;$ for $E_{0}=3 \mathrm{GeV}$ we obtain

$\sigma_{\varepsilon} / E_{0}=8.1 \times 10^{-4}$ and $\sigma_{\tau}=8.1 \times 10^{-11} \mathrm{sec}$.

Let us also note, from Eq. (163) for the bunch length and Eq. (114)

for the synchrotron frequency $\Omega_{s}$, that the bunch length is proportional to

$(\alpha / h)^{1 / 2}$, where $\alpha$ is the momentum compaction, Eq. (75), and $h$ is the harmonic number (104). Short bunch lengths result from large values of time rate of change of cavity voltage and are thus achieved by high $R F$ frequencies and high RF voltages; however, the RF frequency need not be so high if a is small. For example, in the Daresbury storage ring the $R F$ frequency is $500 \mathrm{MHz}$ and $\alpha$ is 0.15 , while for the NSLS x-ray ring the RF frequency is only $50 \mathrm{MHz}$ and $\alpha$ is 0.0065 . Bunch lengths for these two rings are comparable. 


\subsection{Equilibrium Horizontal Beam Size, Effect of Wigglers}

An argument similar to that used to treat photon emission effects on

synchrotron oscillations can be used to determine effects on the equilibrium horizontal beam size. We begin with the Courant-Snyder amplitude, invariant in the absence of photon emission,

$$
A_{x}^{2}=\gamma_{x}(s) x^{2}+2 \alpha_{x}(s) x x^{\prime}+\beta_{x}(s) x^{\prime 2} .
$$

Upon the emission of a photon of energy $u$, we see that

$$
\begin{aligned}
& A_{x}^{2}+\delta A_{x}^{2}=\gamma_{x}(s)\left(x-\eta(s) \frac{u}{E_{0}}\right)^{2} \\
& +2 \alpha_{x}(s)\left(x-\eta(s) \frac{u}{E_{0}}\right)\left(x^{\prime}-n^{\prime}(s) \frac{u}{E_{0}}\right) \\
& +\beta_{x}(s)\left(x^{\prime}-n^{\prime}(s) \frac{u}{E_{0}}\right)^{2},
\end{aligned}
$$

so that

$$
\left\langle\delta A_{x}^{2}\right\rangle=\frac{\left\langle u^{2}\right\rangle}{E_{0}^{2}} \mathcal{H}(s)
$$

with

$$
\mathcal{F}(s)=\gamma_{x} n^{2}+2 \alpha_{x} n n^{\prime}+\beta_{x} n^{\prime 2} .
$$

Equilibrium is determined by

$$
\frac{d<A_{x}^{2}>}{d t}=\frac{1}{2 \pi R E_{0}{ }^{2}}\left\{\oint d s N<u^{2}>\mathcal{H}(s)\right\}-\frac{\left.2<A_{x}^{2}\right\rangle}{\tau x}=0 ;
$$

and, If we use the same substitutiong as were used to obtain (165a), we find that 


$$
\varepsilon_{x} \equiv \frac{\sigma_{x}^{2}(s)}{B_{x}(s)}=\frac{1}{2}\left\langle A_{x}^{2}\right\rangle=\frac{c_{g} \gamma_{0}^{2} \oint d s\left|G^{3}\right| \mathcal{H}}{J_{x} \oint d s G^{2}},
$$

where the equilibrium horizontal emittance $\varepsilon_{x}$ is an invariant, independent of the azimuthal position.

Let us consider the effect of a high field wiggler on the horizontal emittance [See e.g. Kose (1977]. We take as before $G_{0}(s)$ to be the inverse radius of curvature in bending magnets and $G_{w}$ that in the wiggler field, where $\int G_{w}(s) d s=0$. If the bending magnets are isomagnetic and if a In the wiggler, we have purely vertical fields with I/o values $\pm G_{w}$, then the emittance $\varepsilon_{x w}$ with the wiggler on, 1 , in analogy with Eq. $(170)$,

$$
\varepsilon_{x w}=\frac{c_{q} \gamma_{0}^{2}\left[G_{0}^{3} \int_{j a g} g+\left|G_{w}^{3}\right| \int_{w} q j\right.}{J_{x}\left[G_{0}^{2}\left[\frac{2 \pi}{G_{0}}\right]+G_{w}^{2} \ell_{w}\right]},
$$

where $l_{w}$ is the length of orbit in the wiggler and $\left(2 \pi / G_{0}\right)$ is the length in the bending magnets. The ratio of the emittance with wiggler on to that with wiggler off is

$$
\frac{\varepsilon_{\mathrm{ww}}}{\varepsilon_{\mathrm{x}}}=\frac{1+\left(\frac{\rho_{0}}{\rho_{\mathrm{w}}}\right)^{2}\left(\frac{l_{\mathrm{w}}}{2 \pi \rho_{\mathrm{w}}}\right) \frac{\langle\rho\rangle_{\mathrm{w}}}{\langle q\rangle_{\mathrm{Mag}}}}{1+\frac{\rho_{\mathrm{o}}}{\rho_{\mathrm{w}}}\left(\frac{l_{\mathrm{w}}}{2 \pi \rho_{\mathrm{w}}}\right)} .
$$

Suppose the ratio of the dipole bending radius to the wiggler bending radius $\rho_{0} / \rho_{w}=4$, and $\ell_{w} / 2 \pi \rho_{w}=0.1$, then

$$
\frac{\varepsilon_{\mathrm{XW}}}{\varepsilon_{\mathrm{x}}}=\frac{1+1.6 \frac{\langle q\rangle_{\mathrm{W}}}{\langle 9+\rangle_{\mathrm{Mag}}}}{1.4}
$$

If $\langle \%\rangle_{\mathrm{w}} \cong 0$, as is the case if the wiggler is placed at a location where the dispersion is zero, it will act to reduce the emittance, $\varepsilon_{x w} / \varepsilon_{x}=0.7$. 
For a synchrotron light source facility this is desirable; small

emittance leads to high source brightness. On the other hand,

if $\langle 9\rangle_{\mathrm{w}} \approx\langle 9\rangle_{\mathrm{Nag}}$, then the emittance is increased by the wiggler,

$\varepsilon_{x w} / \varepsilon_{x}=1.9$, a result to be desired for a colliding beam macinine, because with

increased horizontal emittance one can obtain larger currents and an increased

beam-beam collision rate. At SPEAR a wiggler was recently installed at a

location with non-zero dispersion. The short wavelength photon spectrum produced

in the relatively high $18-\mathrm{kg}$ field has benefitted synchrotron radiation

users and the concomitant increase achieved in luminosity has been appreciated

by the particle physicists.

\subsection{Vertical Beam Size}

If the vertical dispersion is taken to be zero, the change in the

Courant-Snyder anplitude,

$$
A_{z}^{2}=\gamma_{z}(s) z^{2}+2 \alpha_{z}(s) z z^{\prime}+\beta_{z}(s) z^{\prime 2},
$$

resulting from the emission of a photon of energy $u$ is associated with

the angular deviation $\psi$ of the photon momentum $(u / c)$ from the electron

momentum $\left(E_{0} / c\right)$. At the moment of photon emission,

$$
\delta z=0 \quad \text { and } \quad \delta z^{\prime}=\frac{u}{E_{0}} \cdot \psi
$$


so that

$$
\delta\left\langle A_{z}^{2}\right\rangle=\frac{u^{2}}{E_{0}^{2}} \psi^{2} B_{z}
$$

Equilibrium is determined by

$$
\frac{d\left\langle A_{z}^{2}\right\rangle}{d t}=\frac{1}{2 \pi R}\left(\left\{d s N\left\langle u^{2} \psi^{2}>\frac{B_{z}(s)}{E_{0}^{2}}\right)-\frac{2\left\langle A_{z}^{2}\right\rangle}{\tau_{z}}=0,\right.\right.
$$

and, using

$$
\left\langle u^{2} \psi^{2}\right\rangle \approx\left\langle u^{2}\right\rangle\left\langle\psi^{2}\right\rangle \approx\left\langle u^{2}\right\rangle / 2 \gamma_{0}^{2}
$$

one finds the emittance

$$
\varepsilon_{z} \equiv \frac{\sigma_{z}^{2}(s)}{\beta_{z}(s)}=\frac{\tau_{z}}{16 \pi R_{0}^{2} \gamma_{0}^{2}} \oint N\left\langle u^{2}\right\rangle \beta_{z}(s) d s
$$

It follows that

$$
\varepsilon_{\mathrm{z}} \sim \mathrm{C}_{\mathrm{q}}=3.8 \times 10^{-13} \mathrm{~m}-\mathrm{rad} \text {. }
$$

This is to be compared with

$$
\varepsilon_{x} \sim \gamma_{0}^{2} c_{q}=3.8 \times 10^{-7} \operatorname{m}-\operatorname{rad} \quad\left(\gamma_{0}=1000\right)
$$

Relative to the horizontal emittance, then the natural vertical emittance is negligible; in real machines the dominant contribution to the vertical emittance derives from $x-2$ coupling. One introduces the phenomenological coupling constant $k_{x z}$ in writing the emittance

$$
\varepsilon_{z}=k_{x z}^{2} \varepsilon_{x}
$$

and, because experience with operating machines indicates $k_{x z}$. can be made 
as small as $10^{-1}$, its value is taken to fall between $10^{-1}$ and 1 . If $\varepsilon_{x}^{0}$ is the natural emittance, that is no $x, z$ coupling, then, in the real case when coupling exists, the "oscillation energy" is shared between the $x$ and $z$ modes and the combined emittances must equal $\varepsilon_{x}^{0}$, thus $\varepsilon_{x}+\varepsilon_{z}=\varepsilon_{x}^{0}$. We then have

$$
\begin{aligned}
\varepsilon_{x} & =\frac{\varepsilon_{x}^{0}}{1+k_{x z}^{2}} \\
\text { and } \varepsilon_{z} & =\frac{k_{x z}^{2} \varepsilon_{x}^{0}}{1+k_{x z}^{2}} .
\end{aligned}
$$

\subsection{Quantum Lifetime for Betatron Oscillations}

The equilibrium established between the quantum fluctuations and the

radiation damping results in a Gaussian phase space distribution,

$$
\frac{1}{2 \pi \varepsilon_{x}} e^{-\frac{1}{2 \varepsilon_{x}}\left(\gamma x^{2}+2 \alpha_{x} x^{\prime}+\beta_{x} x^{\prime 2}\right)} d x d x^{\prime}
$$

with

$$
\left\langle x^{2}\right\rangle=\beta_{x} \varepsilon_{x} \text { and }\left\langle x^{\prime 2}\right\rangle=\gamma_{x} \varepsilon_{x}
$$

If we write the Courant-Snyder invariant for $x$ and $x^{\prime}$, 


$$
\begin{aligned}
W_{x}=A_{x}^{2}=Y_{x} x^{2}+2 a_{x} x x^{\prime}+B_{x} x^{\prime 2} \text {, and introduce the change of variables } \\
x=\left(W_{x} B_{x}\right)^{\frac{1}{2}} \cos \phi \\
x^{\prime}=-\left(W_{x} / \beta_{x}\right)^{\frac{3}{2}}\left(\sin \phi+\alpha_{x} \cos \phi\right),
\end{aligned}
$$

then the phase space distribution can be written

$$
D\left(W_{x}, \phi\right) d W_{x} d \phi=\frac{1}{2 \pi<W_{x}>} e^{-W_{x} /<W_{x}>} d W_{x} d \phi,
$$

where $\left\langle\frac{T}{x}\right\rangle=2 \varepsilon_{x}$ and $d x d x^{\prime}=d W_{x} d \phi / 2$.

In a stored beam of $M$ electrons the number having values of $W$ between $w_{x}$ and $w_{x}+d w_{x}$ is simply

$$
M D\left(W_{x}\right) d W_{x}=\frac{M}{\left\langle W_{x}\right\rangle} e^{-W_{x} /\left\langle W_{x}\right\rangle} d W_{x} \text {. }
$$

For a given electron the rate of change of $w_{x}$ due to radiation damping is

$$
\frac{d W_{x}}{d t}=-\frac{2 W_{x}}{\tau_{x}},
$$

which implies that the number of electrons per second whose value of $w_{x}$

decreases through $\hat{\mathrm{w}}_{\mathbf{x}}$ is, given the ideal distribution,

$$
M D\left(\hat{\omega}_{x}\right) \frac{2 \hat{H}_{x}}{\tau_{x}}
$$

At equilibrium this rate of decrease would equal the number of electrons per sec having values of $W_{x}$ which increase through $\hat{w}_{x} \cdot$ However, if, in the 
real situation, $\hat{W}_{x}$ represents the working aperture of the machine, electrons whose values of $W_{x}$ increase beyond $\hat{W}_{x}$ are lost from the beam. Actual loss rates in storage rings are small, that is, an electron makes of the order of $10^{11}$ turns and undergoes perhaps $10^{12}$ oscillations before it is lost. We therefore assume that the equilibrium rates of flux thru $w_{x}$ values in actual rings approximate the the ideal rates.

It follows that the rate of electron loss from the stored beam is

$$
\frac{d M}{d t}=-M D\left(\hat{w}_{x}\right) \frac{2 \hat{w}_{x}}{T_{x}},
$$

and the quantum 1ifetime, $\tau_{q}=\cdots M / \frac{d M}{d t}$, becomes

$$
\tau_{q}=\frac{\tau_{x}}{2} \frac{\left\langle W_{x}\right\rangle}{\hat{W}_{x}} e^{\hat{W}_{x} /\left\langle W_{x}\right\rangle} .
$$

Recall that $\tau_{x}$ is the radial damping time defined in Egs. (135)-(138). Let us assume that the machine aperture is determined at a location In the ring corresponding to a maximum of the $\beta$-function. Then $\hat{W}_{x}=x_{\max }^{2} / \beta_{\max }$ and, with $\left\langle W_{x}\right\rangle=2 \sigma_{x}{ }^{2} / \beta_{\max }$, we obtain

$$
\tau_{q}=\frac{\tau_{x}}{\left(x_{\max } / \sigma_{x}\right)^{2}} e^{\left(x_{\max } / \sigma_{x}\right)^{2} / 2},
$$


where $x_{\max } / \sigma_{x}$ is the number of distribution standard deviations allowed by the aperture. We see that the quantum lifetime varies very rapidly with this number; for $\left(x_{\max } / \sigma_{x}\right)=6, \tau_{q} / \tau_{x}=1.8 \times 10^{6}$ while for $\left(x_{\max } / \sigma_{x}\right)=10$, an aperture typical of machine operating conditions, $\tau_{q} / \tau_{x}=5 \times 10^{19}$. Now $\tau_{x}$ is typically a millisecond from which it follows that the quantum Iffetime in the second case is of the order of $10^{16} \mathrm{sec}$.

\subsection{Energy Aperture}

In the preceding discussion we have confined our attention to the small amplitude synchrotron osctllations describen by the linear Eq. (112). For oscillations of larger amplitude nonlinearities in the RF potential $V(\tau)$ must be taken into account. From Eqs, (109) and (111) we derive

$$
\frac{d^{2} \tau}{d t^{2}}=-\frac{a}{E_{0} T_{0}}\left[e V(\tau)-U_{0}^{j}\right]
$$

where we have neglected the radiation damping by setting $U_{\text {rad }}(\tau)=U_{0}$. We introduce the "potential energy"

$$
\Phi(\tau)=\frac{\alpha}{E_{0} T_{0}} \int_{0}^{\tau} \mathrm{d} \tau\left[\mathrm{\tau}\left[\mathrm{eV}(\tilde{\tau})-U_{0}\right],\right.
$$


and write the first-1ntegral of (190) as

$$
\frac{1}{2}\left[\frac{d \tau}{d t}\right]^{2}+\Phi(\tau)=\Phi_{0}
$$

with $\Phi_{0}$ a constant having a value which depends upon the initial conditions. Using Eq. (109), we see that (192) is equivalent to

$$
\frac{\alpha^{2}}{2}\left[\frac{\varepsilon}{E_{0}}\right]^{2}+\Phi(\tau)=\Phi_{0}
$$

An electron undergoing synchrotron oscillations is confined to the phase space trajectory of (193), with constant $\Phi_{0} \cdot$ If the RF potential has the form of Eq. $(110)$, 1.e. $V(i)=\hat{v} \sin \left(\omega_{r f} \tau+\phi_{\delta}\right)$, the potential energy becomes

$$
\Phi(\tau)=-\frac{e \theta_{\alpha}}{E_{0} T_{0}^{\omega} \mathrm{rf}}\left[\cos \left(\omega_{\mathrm{rf}} \tau+\phi_{s}\right)-\cos \phi_{S}+\omega_{\mathrm{rf}} \tau \sin \phi_{s}\right],
$$

the function sketched In Fig. 17. Clearly, the first term in the bracket produces the oscillation and the third the downward slope. The maximum amplitude of stable oscillation corresponds to $\Phi_{0}=\Phi_{\max }$, as indicated in the figure, with the time displacement bounded by $\tau_{1}$ and $\tau_{2} \cdot$ It follows from (194) that the local maximum of the potential energy corresponds to $\tau_{1}=\left(\pi-2 \phi_{g}\right) / \omega_{\mathrm{rf}}$; hence 
$\ddots$

(
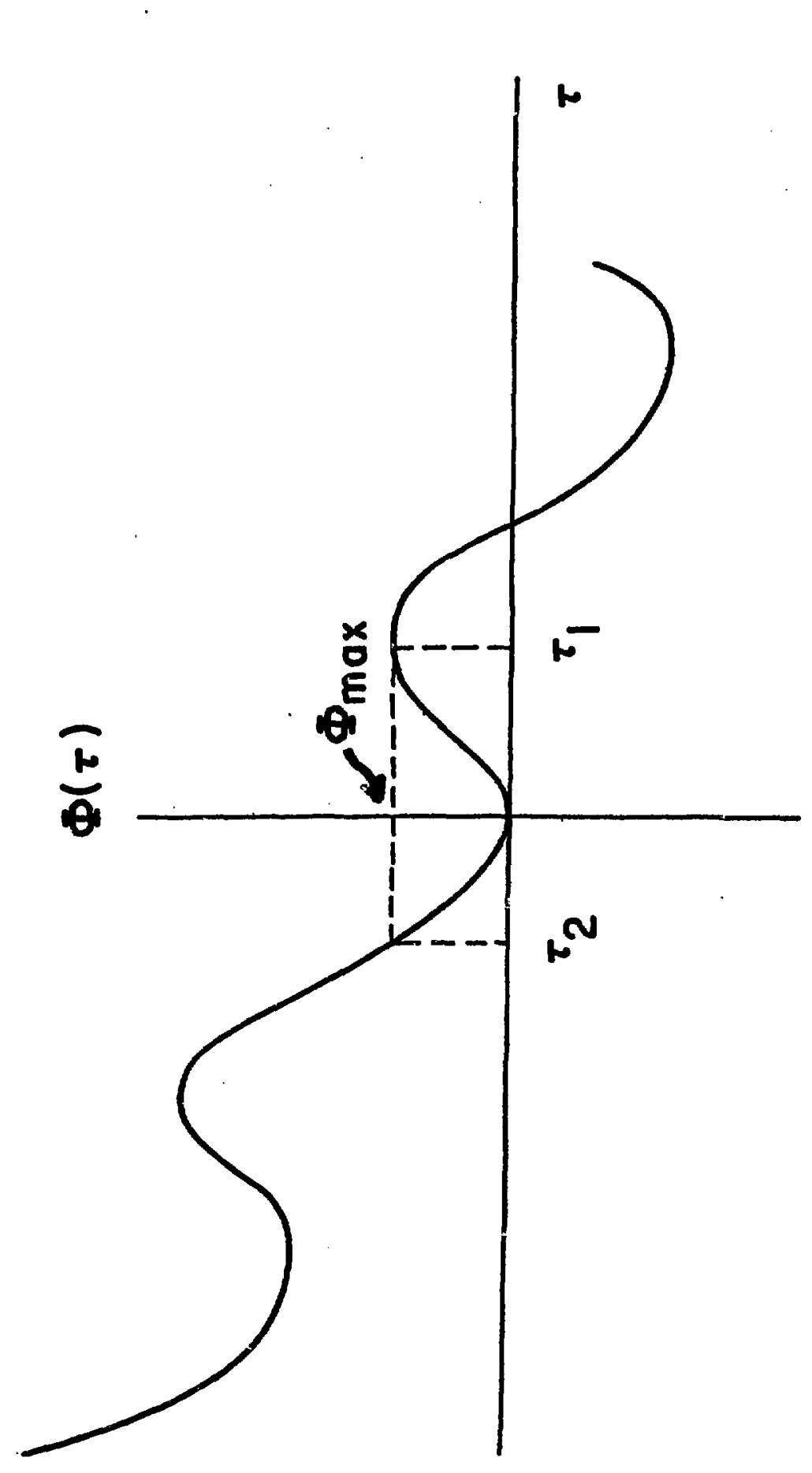


$$
\Phi_{\max }=\Phi\left(\tau_{1}\right)=\frac{\alpha U_{0}}{E_{0} T_{0} \omega_{r f}} F\left(\frac{e \hat{v}}{U_{0}}\right)
$$

with

$$
F(q) \equiv 2\left[\sqrt{q^{2}-1}-\cos ^{-1} \frac{1}{q}\right] \text {. }
$$

where $e f / U_{0}$ is called the overvoltage. In $\varepsilon-\tau$ phase space, stable oscillations occur only inside the fish-shaped separatrix shown in Fig. 18. The maximum energy deviation, $\varepsilon_{\max }$, for which the oscillation can be stabie, the "energy aperture", corresponds to $\Phi_{0}=\Phi_{\max }$ and $\tau=0$, henze from Egs. (193) and (195) we obtain

$$
\left(\frac{\varepsilon_{\max }}{E_{0}}\right)^{2}=\frac{U_{o}}{\pi \alpha E_{o} h} F\left(\frac{e \hat{V}}{U_{o}}\right) \text {, }
$$

where we have set $\omega_{\text {rf }}=2 \pi h / T_{0}$, with $h$ the harmonic number.

\subsection{Quantum Lifetime for Synchrotron Oscillations}

The quantum lifetime associated with synch rotron oscillations may be treated in a way analogous to that used for betatron oscillations in Section 3.16. Neglecting the nonlinearity of the RF potential and ignoring quantum fluctuations and radtation damping, we have written in Eq. (155) an invariant of the synchrotron motion:

$$
w_{s}=A_{s}^{2}=\varepsilon^{2}+\left(\frac{1}{n_{s}} \frac{d \varepsilon}{d t}\right)^{2} \text {. }
$$

With effects of quantum fluctuations and radiation damping included, one obtains the equilibrium phase space distribution 


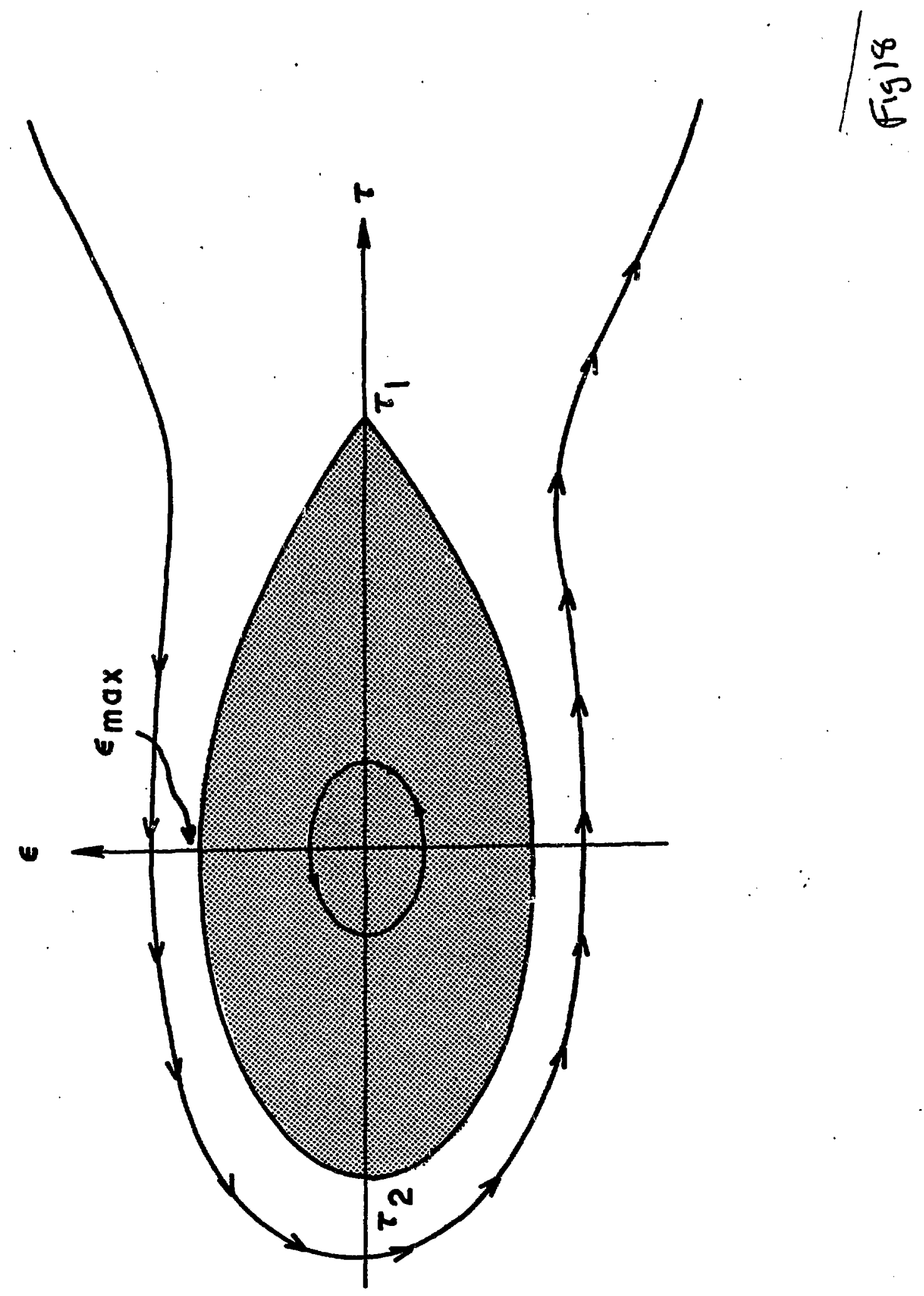




$$
\frac{1}{2 \pi \sigma_{\varepsilon}{ }^{2}} e^{-\frac{1}{2 \sigma_{\varepsilon}{ }^{2}}\left[\varepsilon^{2}+\left(\frac{1}{\Omega_{s}} \frac{d \varepsilon}{d t}\right)^{2}\right]} d \varepsilon d\left(\frac{1}{\Omega_{B}} \frac{d \varepsilon}{d t}\right) \text {. }
$$

An argument analogous to that leading from Eq. (181) to Eq. (184) implies that in a bunch of $M$ electrons the number having values of $W$ between $W_{s}$ and $\mathrm{w}_{s}+\mathrm{dW}$ is

$$
\frac{M}{2 \sigma_{\varepsilon}^{2}} e^{-W_{s} / 2 \sigma_{\varepsilon}^{2}} d w_{s}
$$

ard

Then nonlinearity in the RF potential is taken into account $W_{B}$ is no longer an invariant, and one has instead the invariant $\Phi_{0}$ of $\mathrm{gq}$. (193). If we define $W$ by

$$
\Phi_{0}-\frac{a^{2}}{2 E_{0}^{2}} w
$$

then $W \rightarrow W_{s}$ in the limit of vanishing nonlinearity. For values of $w$ that are not too large, the $W$ distribution should be

$$
\frac{M}{2 \sigma_{\varepsilon}^{2}} e^{-W / 2 \sigma_{\varepsilon}^{2}} d W
$$

Now we know that electrons with values of $w$ larger than $\hat{w}=\left(2 E_{0}{ }^{2} / \alpha^{2}\right) \Phi_{\max }=\varepsilon_{\max }{ }^{2}$ are lost from the beam, and the argument leading from Eq. (184) to Eq. (189) shows that the quantum lifetime for synchrotron oscillation is

$$
\tau_{s}=\frac{\tau_{\varepsilon} \sigma_{\varepsilon}^{2}}{\varepsilon_{\max }^{2}} \mathrm{e}^{\varepsilon_{\max }^{2} / 2 \sigma_{\varepsilon}^{2}},
$$

with $\tau_{\varepsilon}$ given in Eqs. (135)-(138). 
The quantity $\varepsilon_{\max } / \sigma_{\varepsilon}$ depends on the RF peak voltage, $\hat{v}$, a typical ralue of the ratio being 7 . With $T_{\varepsilon} i 1$ millisecond, $\tau_{s}$ is about $10^{6} \mathrm{sec}$. If the NSLS ring is operated at the maximum possible peak voltage, as described in Sect. 3.21, $\varepsilon_{\max } / \sigma_{\varepsilon}$ is much larger; thus, the electron beam occupies only a tiny fraction of the allowed $\varepsilon-\tau$ phase space (Fig. 18) and the synchrotron Iffetime would exceed that of the universe.

$\tau_{q}$ and $\tau_{s}$ are both long, thus other factors generally determine the lifetime of the beam in a storage ring. These include scattering from the residual gas in the vacuum chamber and the Touschek 1ifetime discussed below.

\subsection{The Touschek Lifetime}

When two electrons in a bunch scatter via their mutual Coulomb repulsion, transverse betatron momentum is converted to longitudinal momentum. The scattered electrons will be lost from the beam if their new longt.tudinal momenta fall beyond the acceptance of the RF system. This loss mechanism, known as the Touschek effect, is often dominant in determining the length of time that a high density beam of low-energy electrons may be maintained in a storage ring. A clear discussion of the Touschek lifetime is given in Chapter XXX of Bruck (1966) and here we shall present only the essential features of the calculation.

Consider a frame, $\tilde{F}$, moving with the average velocity of the bunch, and assume that the transverse electron momenta, associated with betatron oscillations, are non-relativistic in this frame. In general, longitudinal velocities in the frame $\tilde{F}$ are small compared with the transverse motion. In the center of mass of two electrons about to 
collide, they have equal and opposite transverse momenta $\pm q$. After scattering, one electron has longitudinal momentum component $+q \cos \theta$, the other a component $-q \cos \theta$, where $\theta$ is the angle, measured in the center of mass Srame, that the two electrons make with respect to the longitudinal direction. The momenta become $\pm \gamma q \cos \theta$ as observed in the laboratory frame $F$, and the electrons are lost from the beam if

$$
r|q \cos \theta|>\Delta \mathrm{p}_{\max }
$$

where $\Delta \mathrm{p}_{\max }$, th: maximum acceptable momentum deviation, corresponds to the energy aperture, Eq. (196). The Touschek process involves knocking the electrons out of the fish shaped area of $\varepsilon-\tau$ stability indicated in Fig. 18. Once outside, the electrons follow trajectories such as that sketched, which generally go in the direction of decreasing electron energy. Hence, even the electron which initially gains energy in the scattering event such that it is oncside the region of stability soon finds itself too low in energy and lost from the beam.

An element of volume $d V$, in the moving frame $\widetilde{F}$, containing $d N$ electrons undergoes loss corresponding to the rate of change

$$
d_{\tilde{N}}=-\overline{v \sigma} \rho d N
$$

Fore, $v=2 q / m$ is the relative velocity of the two electrons, $\rho$ is the electron density $d \mathrm{~N} / \mathrm{dV}$, and $\sigma$ is the total cross-section for loss. This cross-section is an integral over the differential cross-section for Coulomb scattering,

$$
\sigma=\int_{0}^{\cos ^{-1} \mu} d \theta \sin \theta \int_{0}^{2 \pi} d \phi\left[\frac{d \sigma}{d \Omega}\right]_{M \$ 11 e r},
$$


where $\mu=\Delta p_{\max } / \gamma q$ and $\phi$ is the azimuthal angle of the scattered electron. The average $\overline{v \sigma}$ over the betatron momentum distribution is computed in Bruck (1966) and we shall not evaluate it here. If $\overline{v \sigma}$ is taken to be Independent of position in the bunch, the loss rate for the entire bunch $B$, as observed in the moving frame $\widetilde{F}$, is

$$
\dot{N}_{\tilde{E}}=-\overline{v \sigma} \int_{B} \rho^{2} d V
$$

In the laboratory frame $F$, the density at any point in the bunch

$1 s$

$$
-\left[\frac{x^{2}}{2 \sigma_{x}^{2}}+\frac{z^{2}}{2 \sigma_{z}^{2}}+\frac{L^{2}}{2 \sigma_{L}^{2}}\right]
$$

so that

$$
\int_{B} \rho^{2} d V=\frac{V^{2}}{V_{B}}
$$

with

$$
v_{B}=(4 \pi)^{3 / 2} \sigma_{x z} \sigma_{L} \sigma_{L}
$$

In the moving frame $\widetilde{\mathrm{F}}$,

$$
\int_{B} \rho^{2} d V=\frac{N^{2}}{r V_{B}}
$$

and the loss rate, from Eq. (206), thus corresponds to

$$
\dot{N}_{\tilde{F}}=-\overline{v \sigma} \frac{N^{2}}{r V_{B}}
$$


Transforming back to the lab, we find

$$
\dot{\mathrm{N}}=-\left\{\frac{\overline{\mathrm{v \sigma}}}{\gamma^{2} \mathrm{v}_{B}}\right\} \mathrm{N}^{2}
$$

It then follows that

$$
N(t)=\frac{N_{0}}{1+\left\{\overline{v \sigma} / \gamma^{2} v_{B}\right\} N_{0} t},
$$

so the half-1ife $T_{\frac{1}{2}}$ is

$$
\frac{1}{\tau_{1 / 2}}=N_{0}\left\{\frac{\overline{v \sigma}}{\gamma^{2} v_{B}}\right\}
$$

For a ribbon-like beam, in which the vertical betatron oscillations are negligible relative to the horizontal, one finds (Bruck, 1966)

$$
\frac{1}{\tau_{3 / 2}}=\frac{\sqrt{\pi} r_{0} c N C(\xi)}{\sigma_{x} \cdot \gamma^{3}\left(\varepsilon_{\max } / E_{0}\right)^{2} v_{B}}
$$

where $r_{0}=2.8 \times 10^{-15} \mathrm{~m}, \sigma_{x^{\prime}}$ is the one-standard deviation horizontal angular spread, $\varepsilon_{\max } / E_{0}$ is the maximum relative energy deviation accepted by the RF [given in $\mathrm{Eq} .(196)$ ],

$$
\xi \equiv\left[\frac{\varepsilon_{\max } / E_{0}}{\gamma \sigma_{x^{\prime}}}\right]^{2}
$$

and $c(\xi)$ is a function given in Bruck, which for $\xi<10^{-2}$ is well approzimated by 


$$
c(\xi)=\ln \left(\frac{1}{1.78 \xi}\right)-1.5
$$

It may be noted that the instantaneous value of $\tau_{\frac{1}{2}}$ changes, as the bunch traverses the orbit, with change in $\sigma_{x}$, and $\nabla_{B}$ of Eq. (215); the actual Touschek Iifetime is perforce an average.

As an illustration of the Importance of the Touschek effect in dissipating the circulating beam, we may consider the NSLS $x$-ray ring when operating at $2.5 \mathrm{GeV}$ and $0.5 \mathrm{amp}$. From Eq. (215) with the parameters given in Section 3.21, we find $\tau_{\frac{3}{2}}$ to be about 30 hours. If, at lower beam energies, $\sigma_{x} \sigma_{2}$ and $\sigma_{x}$ scale according to ideal behavior, In proportion to energy, the Touschek 11fetime at injection energy $0.7 \mathrm{GeV}$ will be about one minute. In actuality these $\sigma^{\prime} s$ are not so small at $: \cdot i$ injection and a lifetime at injection of the order 10 minutes is expected. This is adequate for successful loading of a high current into such a machine. High current, high brightness rings, intended for $0.7-$ to $1-\mathrm{GeV}$ operation, are under construction at several laboratortes. These have electron densities, $N / V_{B}$, which are similar to that of the $2.5-\mathrm{GeV}$ machine cited above. By operating with greater over voltages, $\varepsilon_{\max } / \mathrm{E}_{0}$ is larger [see Eqs. (195) and (196)] and Touschek Iifetimes of several hours should be attained.

\subsection{Coherent Instabilities}

A high intensity beam of electrons in a storage ring may behave differently from a single particle whose motion is determined entirely by the magnetic guide field. Historically, as attempts have been made 
to Increase the current circulating, it has been necessary to identify and eliminate a series of coherent instabilities. These are caused by the electromagnetic Interaction between the beam and the material structures that surround it, such as RF cavities, bellows, injection equipment, and the vacuum chamber. itself. The beam current generates electromagnetic fields in these structural elexents which act back on the beam and may cause amplification of certain coherent beam oscillation modes. The result can be loss of the beam or dilution of the bunch density via bunch lengthening or widening.

In general, one is not troubled by a particular instability until a certain threshold current is exceeded. For a bunched beam, it is often the peak current which enters the stability criterla, and hence for a given number of electrons, such instabilities cause more trouble the shorter the bunch. One may classify instabilities as longitudinal or transverse, and as being associated with single or multibunch behavior. An example of a multibunch Instability is the interaction of the beam with a high-Q cavity; if the field set up in it by one passing bunch decays slowly, there will be interaction with succeeding bunches as they pass by. The "head-tail" Instability is an example of a single bunch phenomenon in that fields set up by the particles in the front of the bunch act on the particles in the rear. This head-tail interaction can lead to instability because particles are continually moying between the front and rear of the bunch due to the synchrotron oscillations.

In designing a storaze ring one attempts to construct a vacuum enclosure having a minimum of abrupt cross-sectional changes. 
During the early operation of a machine, one works to damp troublesome modes that appear in various structural elements despite the best efforts of the designers. Remaining Instabilities are usually managed by Landau damping or by feedback systems. We remark that Landau damping results from the presence of a range of synchrotron frequencies and betatron tunes in the electron beam. In the absence of a spread in the oscillation frequencies, an external disturbance readily drives a single coherent node to large amplitude. When there exists a frequency spread, the oscillation energy of the coherently driven mode is dissipated among many coupled, but incoherent, modes. One might say that Landau damping results from incoherent stability countering coherent instability. In practice the spread in betatron tunes may be Increased by Inclusion of octupole magnets as part of the lattice; these introduce betatron oscillation frequencies which are amplitude dependent and Incoherent. One may increase the spread of synchrotron frequencies by Including an $R F$ cavity operating at some harmonic, say the fourth, of the basic RF frequency. Furthermore, different bunches can be given different tune values by use of an RF quadrupole. 


\subsection{Exanple: NSLS 2.5-GeV Storage Ring}

The optical propertles of a synchrotron light source are governed by many storage ring parameters and it may be useful to close this section by examining a set of parameters for a yarticular storage ring. For reasons of their own conventence, the authors have chosen for this 11lustration the $2.5-\mathrm{GeV}$ ring under construction at Brookhaven. Let us first list some parameters which are flxed by the machine design and which are not readily varied:

$\begin{array}{lrl}\text { Effective Radius } & R=\text { circumference } / 2 \pi=27.06 \mathrm{~m} \\ \text { Bending Radius } & p=6.875 \mathrm{~m} \\ \text { Orbital Period } & T_{0}=2 \pi \mathrm{R} / \mathrm{c}=0.568 \mu \mathrm{sec} \\ \text { RF Frequency } & v_{\mathrm{rf}}=\omega_{\mathrm{rf}} / 2 \pi=52.88 \mathrm{MHz}, \\ \text { Earmonlc Number } & h=v_{r f} T_{0}=30\end{array}$

Other parameters are subject, in varying degrees, to cholce. Here we consider an operating mode ylelding high brightness at the Insertion centers: that 1s, small values of the amplitude functions $\beta_{x}$ and $\beta_{2}$ (Fig. 14) and small beam sizes $\sigma_{x}$ and $\sigma_{z}(F 18.19)$. Low $B$ values are not always desired, however. When undulators (Section 6) are employed, for example, one wishes to op«rate with small values of the electron angular deviations $\sigma_{x^{\prime}}$ and $\sigma_{z^{\prime}}$; these conditions and others as well may be oitained by changing the focusing strengths of the quadrupoles. For the mode with low $\beta$ at the insertions: 


$$
\begin{aligned}
& \text { Amplituci Functions }{ }_{x}^{\beta}(s),{ }_{z}^{\beta}(s) \\
& \text { [Eqs. (78)-(87)] } \\
& \text { Minimum } \beta \text {-values } \quad \beta_{x} \cong 1 m, \beta_{z} \approx 0.3 m \\
& \text { Maximum B-values } \beta_{x} \cong 26 \mathrm{~m}, B_{z} \cong 28 \mathrm{~m}
\end{aligned}
$$$$
\text { Bee F18. } 14
$$

Dispersion Function $n(s)$

[Eq. (72)]

$$
\text { Maximum } n \text {-value } n \equiv 1.4 \mathrm{~m}
$$

Tunes $\quad v_{x} \cong 9.7, v_{z} \cong 5.7$

[Eqs. (78b), (100), (101)]

Trangverse spatial and angular deviations of electron beam

$$
\sigma_{x}, \sigma_{x}, \sigma_{z}, \sigma_{z}, \quad \text { see Fig. } 19
$$

Momentum Compaction $\alpha=6.5 \times 10^{-3}$

[Eq. (75), (76)]

Damping Partion Numbers $\mathrm{J}_{\mathrm{x}}=1-\mathcal{N}, \mathrm{J}_{\mathrm{z}}=1, \mathrm{~J}_{\varepsilon}=2+\mathscr{W}$

[Eqqs. (135)-(138)]

$$
\theta=\frac{\alpha R}{\rho}=0.025
$$

[Eq. (122)]

Next we consider several parameters which are strongly correlated with the electron beam energy. Values are tabulated for the operating energy $E_{0}=2.5 \mathrm{GeV}(\gamma=4900)$ and for the infection energy $E_{0}=$ $0.7 \mathrm{GeV}(\gamma=1370)$ : 


\section{$2.5 \mathrm{GeV}$}

800

RF Peak Voltage $\hat{v}(k v)$

[Eqs. (103), (110)]

Energy Loss per Turn $U_{0}(k e V)$

503

[Eq. (4a)]

Synchronous Phase $\phi_{\mathbf{S}}$ (deg)

39

3.4

[Eq. (105)]

Damping Times $2 \tau_{\varepsilon} \approx \tau_{x} \cong \tau_{z}$ (msec)

5.6

260

[Eq. (135), $\tau_{1}=1 / \alpha_{1}$ ]

Energy Spread $\sigma_{\varepsilon} / E_{0}$

$8.2 \times 10^{-4}$

$2.3 \times 10^{-4}$

[Eq . (166)]

Synchrotron Oscillation Period $T_{\varepsilon}=2 \pi / \Omega_{s}$ (msec)

0.20

0.38

[Eq. (114)]

Energy Aperture $\varepsilon_{\max } / E_{0}$

0.015

0.015

[Eq. (196)]

Bunch Length $\sigma_{L}=c \sigma_{\tau}(\mathrm{cm})$

5.0

[Eq. (163)]

Horizontal Emittance $\varepsilon_{x}$ (m-rad)

$8 \times 10^{-8}$

$7 \times 10^{-9}$

[Eq. (170)]

Vertical Emittance $\varepsilon_{z} \cong \varepsilon_{x} / 100$

$8 \times 10^{-10}$

$7 \times 10^{-11}$

[Eq. (180), assuming 10\% coupling]

The RF peak voltage is chosen in the above example to give an energy aperture of $1.5 \%$. In practice, the actual peak voltage may be somewhat different, but the numbers given should be representative. Operation of a 
storage ring is wost difficult at the injection energy, the condition under which danping timas are longest and effects such as coherent Instabilities, Touschek Intra-bunch scattering, and scattering by residual gas Ions are the most troublesome. As a result, the very small bunch dimensions quoted above for $0.7 \mathrm{Gev}$ are not expected to be realized in the actual machine; the effects just mentioned tend to "blow-up" the beam and may even cause 1088 of electrons from the bunch. The user of a synchrotron 1ight facility is not directly interested the properties of the eiectron beam at infection, but he certainly is affected by the maximum achievable stored current, and this may be determined at Injection. Experience at operating storage rings ind1cates that the values for the bunch dimensions and energy spread given above for $2.5 \mathrm{GeV}$ should be reliable approximations. 


\section{OPTICAL CHARACTERISTICS OF THE SOURCE}

\subsection{Introduction}

In this section we consider the optical characteristics of a synchrotron 1Ight source, which depend on the spatial distribution of the electrons in orblt as well as on the spatial character of the emissicial process itself. It was seen in the previous section that the distributions of electrong in $x$ and $z$ position and In $x^{\prime}$ and $z^{\prime}$ angles about the ideal orbit have Gausstan forms. Also, the variation in intensity with angle of the parallel polarized component of emission from a single electron is approximately Gaussian, as suggested in Fig. 3. Generally, the use of Gaussians to represent the emission distributions greatly simplifiesdiscussion of the optical properties of synchrotron radiation sources. With this simplification we will compare the contributions of the emission process and of the electron orbit parameters to the effective source size. The source is small in its $x, x^{\prime}, z$ and $z^{\prime}$ dimensions, (Fig. 19) but it is, of course, extended longitudinally along the orbit direction. This has optical consequences to be deduced by use of the transfer matrix techniques of the foregoing section; the extended source can be represented as an effective source lying In a plane normal to the orbit at some polnt. Source "brightness", which is photon flux per unit source area and unit solid angle of emission, must be high for experiments requiring both spatial and angular resolution, as is the case in an accurate scattering measurement of associated energy and momentum transfer. Further, it will be shown that a short source segment has nearly the same central brightness as an extended one. After 


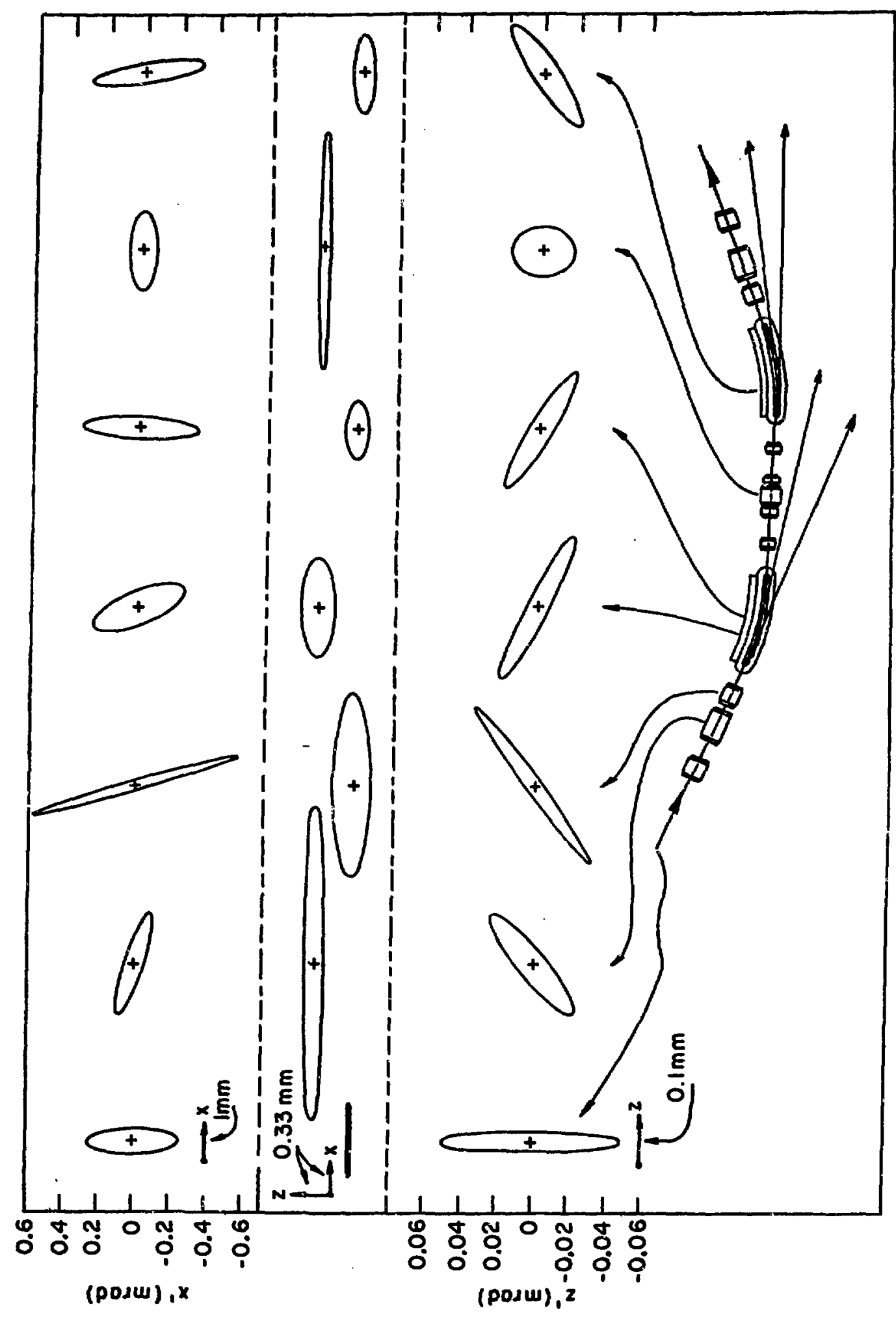

$\mid \frac{\frac{0}{w}}{w^{2}}$ 
considering source characteristics, we will present a few simple examples of the results of coupling the source to optical elements.

This Section 4 is based largely on an important unpublished paper of Green (1976), In which more detail may be found.

\subsection{The Gaussian Approximation to Synchrotron Light Emission}

A probability distribution $P(y)$, where $y$ is a displacement from the most probable value of a coordinate, is of Gausstan form if

$$
P(y)=\frac{1}{\sqrt{2 \pi} \sigma_{y}} e^{-y^{2} / 2 \sigma_{y}^{2}},
$$

where the prefactor normalizes the total probability to unity over the range of $y$ from to to $-\infty$. Of the whole distribution $68 \%$ is associated with the $y$ range between $\sigma_{y}$ and $-\sigma_{y}$ and $95 \%$ for that between $\pm 2 \sigma_{y}$. We have seen that the vertical $(x)$ as well as the horizontal ( $x$ ) phase space distributions of electrons can be described in terms of elliptical contours. The $1 \sigma$ contour is

$$
\frac{y^{2}}{\sigma_{y}^{2}}-\frac{2 x y y^{\prime}}{\sigma_{y^{\sigma} y^{\prime}}}+\frac{\left(y^{\prime}\right)^{2}}{\sigma_{y^{\prime}}{ }^{2}}=\left(1-x^{2}\right), \quad y=x \text { or } z,
$$

where $r$ is an asymetry parameter; ellipse axes are parallel to $y$ and $y^{\text {: }}$ if $r=0$. The fraction of an electron beam within the lo ellipse is 0.39 and for a $2 \sigma$ ellipse the fraction is 0.865 . If we describe the angular intensity dependence of synchrotron radiation from 
a single electron by a Gaussian having angular width parameter $\sigma_{R}$, then the angular divergence of the optical source at some point along the orbit is described by

$$
\sigma_{Y^{\prime}}=\sqrt{\sigma_{y^{\prime}}^{2}+\sigma_{R}^{2}} \quad y^{\prime}=x^{\prime} \text { or } z^{\prime} \text {. }
$$

In IInear dimension, helght and width, the source size is simply $\sigma_{y^{\prime}}$ the electron bunch size. Although one might expect that the central source brightness would be proportional in general to the photon flux divided by $\left(\sigma_{\mathrm{x}} \cdot \sigma_{\mathrm{X}},{ }^{\cdot} \sigma_{\mathrm{z}} \cdot \sigma_{\mathrm{Z}},\right)$, this turns out not to be the case due to the longitudinal extension of the source along the orbit.

Green (1976) has shown and inspection of FIg. 3 suggests that the varlation in intensity, with vertical angle, of the parallel component of light emission from an electron is well described by a Gaussian if $\lambda / \lambda_{c}$ is not more than about 30. Inasmuch as this is the princlpal component of the radiation and that for which optical design is optimized, we shall focus on it here. Let us assume, then; that the intensity, as a function of vertical emission angle $\psi$ ( $\psi$ defined as in Fig. 6b), is

$$
\mathrm{dN}_{\| 1}(\psi, \lambda)=\frac{g(\lambda)}{\sqrt{2 \pi} \sigma_{R}} e^{-\psi^{2} / 2 \sigma_{R}^{2}} d \psi
$$

Here $g(\lambda) /\left(\sqrt{2 \pi} \sigma_{R}\right)$ can be determined from the value for the $\varepsilon_{\|}$component of Eq. (5I) at $\psi=0$. Note that Eq. (51) must be rewritten as a phoron flux Instead 
of as energy per electron-turn. We then find [Eq. (12)]

$\left[\frac{d N}{d \psi}\right]_{\psi=0}^{d \psi}=\frac{d N_{H}}{d \psi}(0, \lambda) d \psi=\frac{g(\lambda)}{\sqrt{2 \pi \sigma_{R}}}-3.461 \times 10^{4} \mathrm{H}_{2}\left(\lambda_{c} / \lambda\right) \gamma^{2}$,

where

$$
\mathrm{H}_{2}\left(\frac{\lambda_{\mathrm{c}}}{\lambda}\right) \equiv\left(\frac{\lambda^{\lambda}}{\lambda}\right)^{2}\left[\mathrm{~K}_{2 / 3}\left(\frac{\mathrm{c}}{2 \lambda}\right)\right]^{2}
$$

and $d x_{y}$ is defined for a milliampere of electron current, a milliradian of the orbit, a milliradian $d \psi$ and a fractional bandwidth, $\Delta \lambda / \lambda$, of 0.01 .

Then $g(\lambda)$ may be determined by integrating Eq. (220) over all angles. $\psi$ and relating the result to the total emission as given by .Eq...(54), which, -like-Eq. (51), is rewritten as a photon flux. One obtains [Eq. (11)]

$$
\int \mathrm{dN}_{\|}(\psi, \lambda) \mathrm{d} \psi=g(\lambda)=1.256 \times 10^{8} \gamma \mathrm{f}^{-} \ddot{G}_{1}\left(\lambda_{c} / \lambda\right),
$$

where $f$ is the fraction of parallel polarization at the wavelength in question and

$$
G_{1}\left(\frac{\lambda_{c}}{\lambda}\right) \equiv\left(\frac{\lambda_{c}}{\lambda}\right) \int_{\lambda_{c} / \lambda}^{\infty} k_{5 / 3}(n) d n .
$$

Eqs. (221) and (223) Imply that

$$
\gamma \sigma_{R}=1.448 \times 10^{3} \frac{f G_{1}}{H_{2}} \text { milliradtans . }
$$

An Important feature of Eq. (225) is that the right-hand side is a function only of $\lambda / \lambda_{c}$. Green (1976) has shown that Eq. (225) is well approximated by the useful relation

$$
\sigma_{R}=565\left(\frac{1}{\gamma}\right)\left(\frac{\lambda}{\lambda_{c}}\right)^{0.425} \text { milliradians. }
$$


Now, what is usually designated as a lo angle subtended at the emitting source is the range from $+\sigma_{R}$ to $-\sigma_{R}$ or $2 \sigma_{R}$; we can see that this angle, at $\lambda=\lambda_{c}$, Is about $1 / \gamma$ radtans, in accord with the results of Section 2 .

\subsection{The Effective Source Size}

It is useful to compare and combine $\sigma_{R}$ of the foregoing subsection wth the $\sigma_{x^{\prime}}$ and $\sigma_{z}$, characteristics of the electrons at various locations in the orbit. Several phase ellipses of the Brookhaven NSLS X-ray ring are plotted in Fig. 19 and values of $\sigma_{x}, \sigma_{x}, \sigma_{z}$ and $\sigma_{z}$ are plotted for the Berlin ring BESSY in Fig. 20. It must be emphasized that, because Figs. 19 and 20 represent design and not performance, it may turn out that they are somewhat optimistic. As noted in Section 3, the vertical beam size arises primarily from coupling of the naturally larger horizontal oscillations into the vertical modes. Fig. 19 is based on an assumed ten percent coupling, 1.e. $k_{x z}=0.1$ in Eqs. (179) and (180). At the Stanford ring SPEAR,significantly smaller coupling than this has been realized. For fig. 20 a relatively pessimistic coupling assumption was made; as a result, $\sigma_{z}$ and $\sigma_{z}$, are not as small, relative to their horizontal eounterparts, as is the case in Fig. 19. At their operating electron energies, $2.5 \mathrm{GeV}$ in NSLS and $0.75 \mathrm{GeV}$ in BESSY, $\sigma_{R}$ values for these two rings for $\lambda=\lambda_{c}$ are $\sim 0.1 \mathrm{mrad}$ and $0.3 \mathrm{mrad}$, respectively. Thus, the figures show that generally

$$
\sigma_{\mathbf{x}}, i \sigma_{\mathrm{R}}
$$

and

$$
\sigma_{\mathbf{z}^{\prime}}<\sigma_{\mathrm{R}}
$$



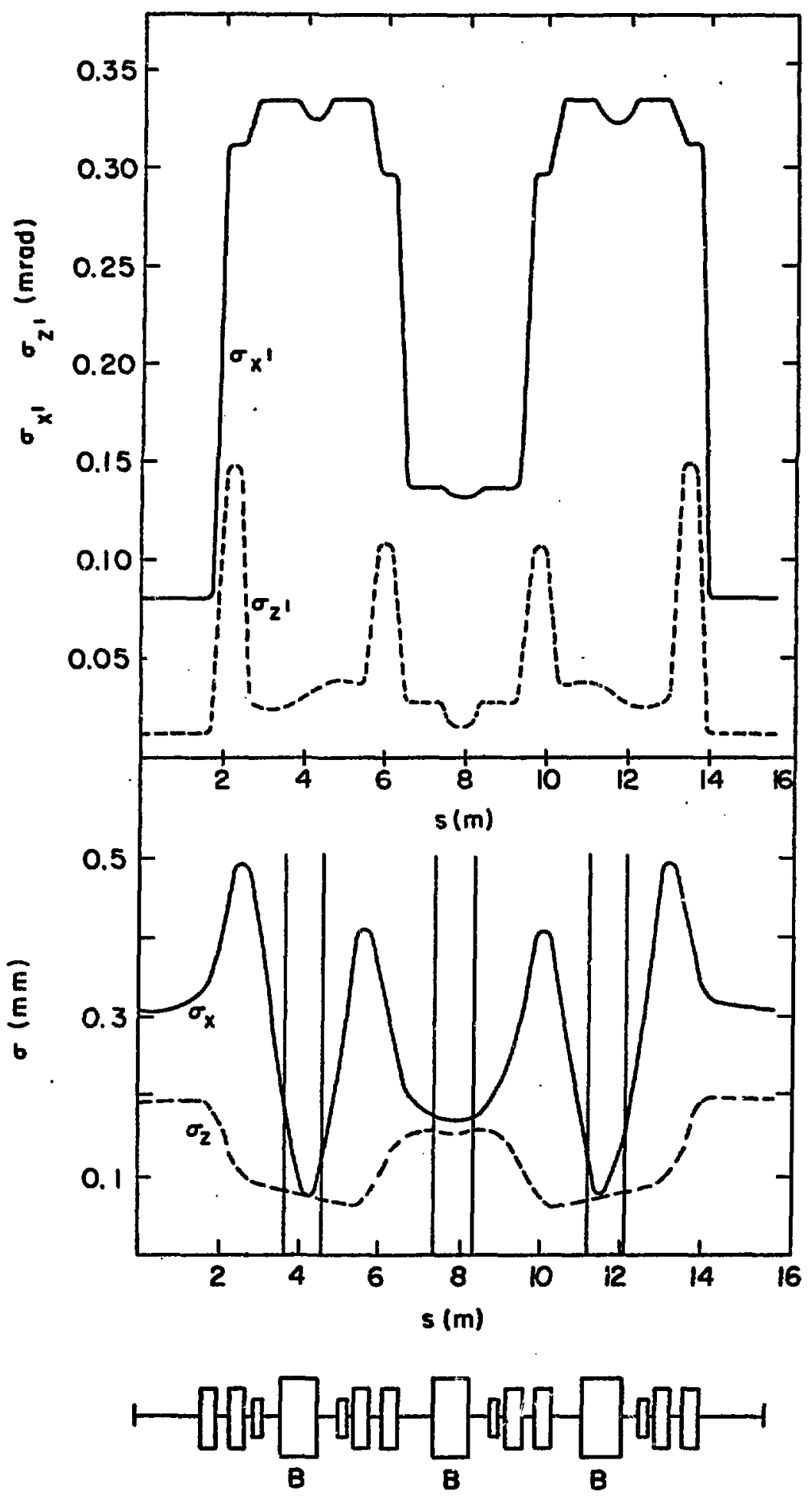
This is a desirable situation: when $\sigma_{x}$, and $\sigma_{z}$, are about equal to or smaller than $\sigma_{R^{\prime}}$, the effective source divergence angles $\sigma_{X}$ and $\sigma_{Z}$, are only slightly larger than the minimum possible, $\sigma_{R}$.

Consider a point along the orbit where the $z, z^{\prime}$ electron ellipse has the symmetrical form of Hig. 21. The photon source, though it has the $z$ dimension of the electrons, is much larger in angular divergence because $\sigma_{R}$ is almost always larger than $\sigma_{2}$, and because these $\sigma^{\prime}$ 's combine to give $\sigma_{Z}$, of $\mathrm{Eq}$. (219). In application one generally collects radiation from a segment of orbit which comprises some distance along the azimuth, say 28, centered on a point. Suppose this point to have the phase space ellipse of Fig. 21 and let us consider the resulting vertical phase space effects for the photon source. If the ellipse is known at one orbit location, it can be obtained at other locations via the transfer matrix methods already described. One can then derive the corresponding photon ellipse and transform it back to the point of origin at the segment center, thus defining the "effective contribution" at the center made by that off-center source element. Thus, if the magnetic field has no focusing properties in the region involved, the electron ellipse of Fig. 21, that of the origin, may be transformed to arrive at the tilted ellipse of Fig. 22a, representing electron phase space at point ts. In Fig. 22b the slightly tilted large ellipse represents the corresponding photon source at +8 . When this ellipse is transformed back to the origin to derive its "effective contribution" as a source element, it is tilted back actually beyond the vertical so that the final extension in $z$ is about $\pm \sqrt{\sigma_{2}{ }^{2}+s^{2} \sigma_{R}{ }^{2}}$, as shown in Fig. 22c. Relative to that of the 


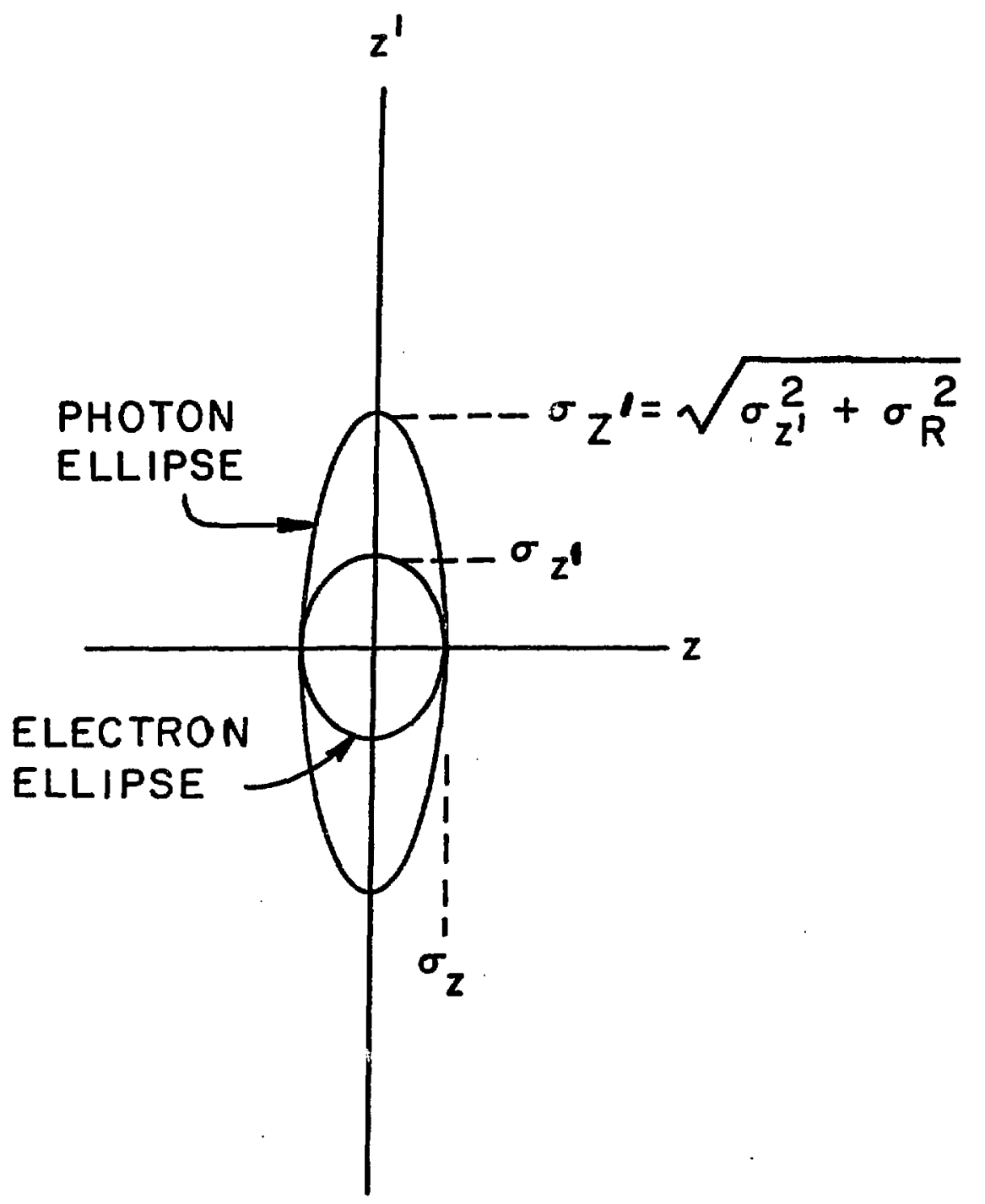




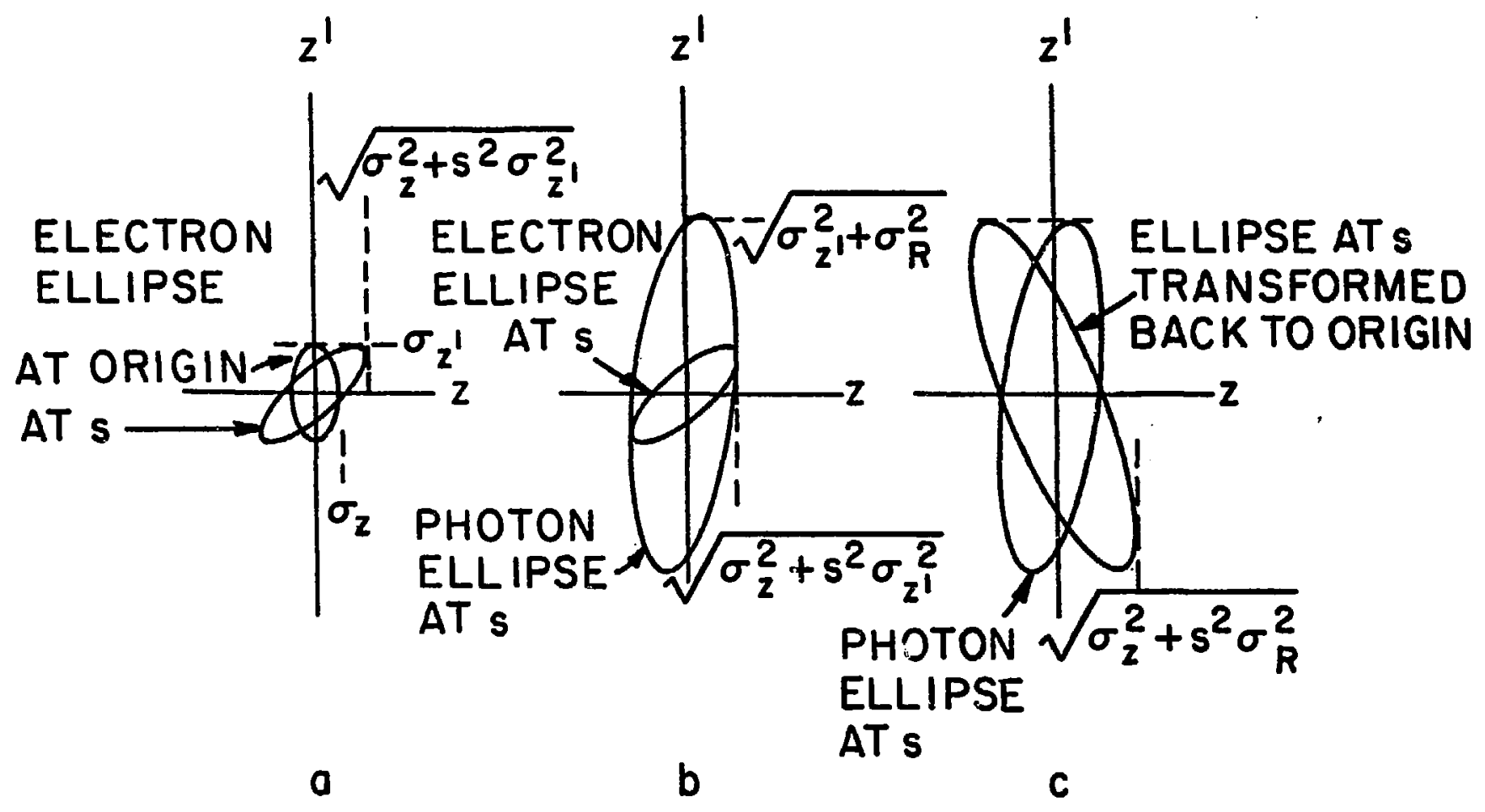


central element, Fig. 21, the contribution of the element at ts has the same $z, z^{\prime}$ center and the same $z^{\prime}$ extent but has considerably greater $z$ extent. The effective phase space for the entire source then may be approximated by suming the contributions of the constituent elements when all have been transformed back to the segment center. If the electron ellipse of the center itself is symetrical about the vertical, as in Fig. 21, the sum for the full source, which comprises paired but oppositely tilted. contributions from elements at positive and negative s values, is symetric in $z$ and $z^{\prime}$. Green (1976), who applied the transfer matrix method to this problem, found that the central photon flux, 1.e. $z=z^{\prime}=0$, for one milliamp of current and one percent bandwidth, is

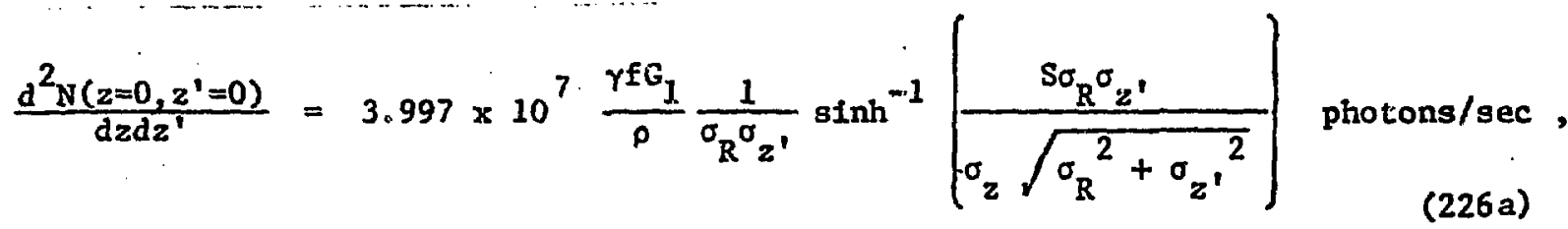

where $\rho$ is in meters and the units otherwise are in and mrad. $S$ is the segment half length and the prefactor, with $G_{1}$, represents the full flux for one milliradian of orbit over the entire vertical angle $\psi$. Eq. (226a) shows that for short sources the central flux varied linearly with $S$, as it must, because $\sinh ^{-1} A \approx A$ for small $A$. For large $A, \sinh ^{-1} A$ increases logarithmically, which means that the $z, z^{\prime}$ brightness is effectively limited for large $S$. This is readily understandable in view of the fact the Io ellipses representing source segments distant from the center have relatively large areas (Fig. 22c) and hence contribute little to the central flux. For a short source Eq. (226a) becomes

$$
\frac{d^{2} N\left(z=0, z^{\prime}=0\right)}{d z d z^{\prime}}=\frac{3.461 \times 10^{4} \gamma^{2} H_{2}}{\sqrt{2 \pi} \sigma_{z}} \cdot \frac{2 S}{\rho},
$$

which could easily have been derlved from Eq. (221) directly. 
In $\left(x, x^{\prime}\right)$, the horizontal phase space, the effects of orbit curvature are more pronounced than is the case in vertical space $\left(z, z^{\prime}\right)$. Consider the geometry shown in Fig. 23, where again the electron ellipse is assumed to be vertical at $s=0$, the segment center. Coordinates of the center of this ellipse are, of course, $x=x^{+}=0$. Coordinates of the centers of ellipses located along the segment on the ideal orbit circle are given by

$$
x^{\prime}=s / \rho \text { for } s \ll \rho
$$

and, for short segments, with the approximation $\cos \theta=1-\theta^{2} / 2$,

$$
x=s^{2} / 2 p
$$

Let us assume that the angular distribution for the radiation process in the horizontal is the same as in the vertical; this is not strictly true, as can be seen from Eq. (48), but it should hold crudely. The lo contour for photons is then approximately

$$
x=\frac{s^{2}}{2 p} \pm \sqrt{\sigma_{x}^{2}+s^{2} \sigma_{R}^{2}}=\frac{\rho x^{\prime 2}}{2} \pm \sqrt{\sigma_{x}^{2}+\sigma_{R}^{2} \rho^{2} x^{2}}
$$

With increasing positive and negative s the ellipses do increase somewhat In size, but the important effect is that their centers are displaced from zero in both the $x$ and $x^{\prime}$ coordinates as seen in Fig. 23b. From Eq. (229) we may determine the probability that a photon emitted at $s$ will pass between $x$ and $x+d x$ at the origin of the orbit segment. It is 


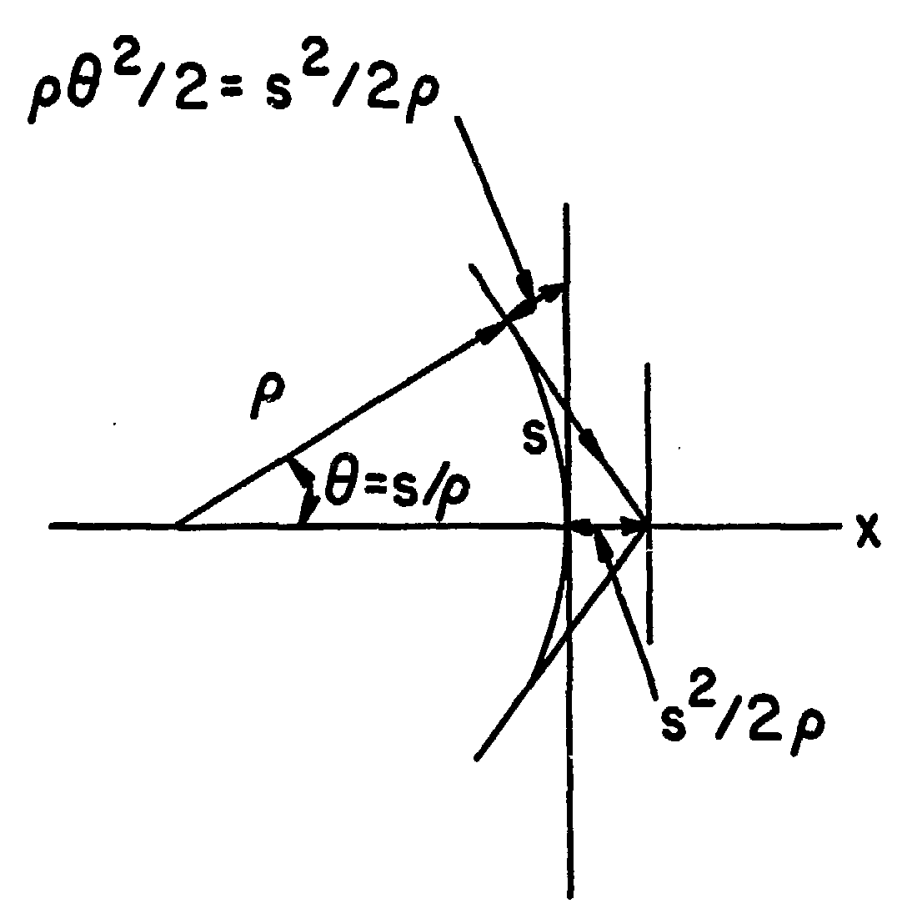

a

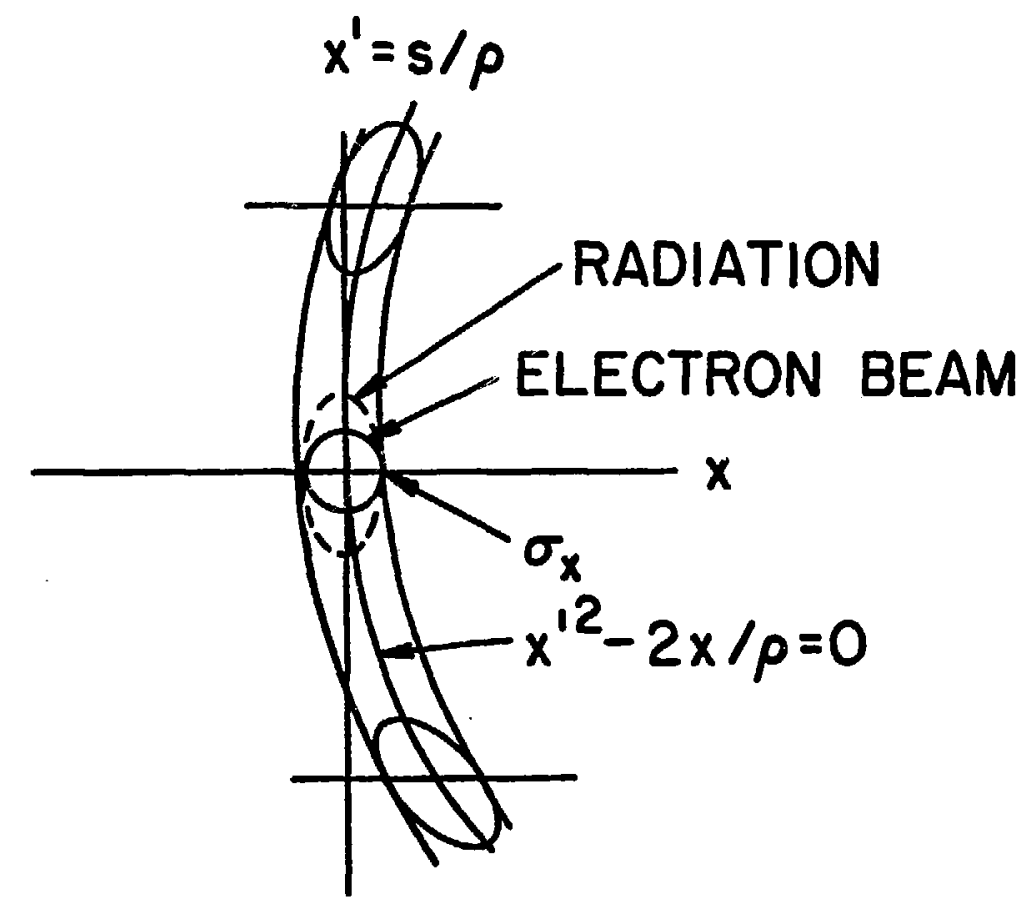

b

Fig23 


$$
P(x)=\frac{d x}{\sqrt{2 \pi} \sqrt{\sigma_{x}^{2}+s^{2} \sigma_{R}{ }^{2}}} e^{-\frac{3}{2} \frac{\left(x-s^{2} / 2 p\right)^{2}}{\sigma_{x}^{2}+s^{2} \sigma_{R}{ }^{2}}} .
$$

One could sum this over a finite orbit segment as was done for the vertical plase space; in practice, however, one often observes orbit segments which are "short" In the sense that the exponential is essentially constant, and in this case the probability that a photon passes through dx at $x=0$ is

$$
P(0)=\frac{d x}{\sqrt{2 \pi} \sigma_{x}}
$$

By multiplying $P(0)$ by the total number of photons enttted per milliradian of orbit [Eq. 223] and by $d x^{\prime}$, we obtain the central density for a short source

$$
d^{2} N\left(x=0, x^{\prime}=0\right)=1.256 \times 10^{8} \gamma f G_{1} \frac{d x d x^{\prime}}{\sqrt{2 \pi} \sigma_{x}}
$$

photons per sec for one percent bandwidth and 1 milliamp current, where $\sigma_{x}$ and $d x$ are in the same units and $d x^{i}$ is in mrad. Unlike the result for the vertical phase space flux, Eq. (226), in Eq. (231) the orbit length does not appear explicitly because of (227) the relationship of $s$ to $x^{\prime}$ and hence $d x^{\top}$. For a short orbit segment, the probability that the photon passes through $\mathrm{dzdz} \mathrm{z}^{\prime}$ at $z=\mathrm{z}^{\prime}=0$ is simply $\mathrm{dzd} \mathrm{z}^{\prime} /\left(2 \pi \sigma_{\mathrm{z}} \sqrt{\sigma_{z^{\prime}}^{2}+\sigma_{R}^{2}}\right)$; thus the central brightness of such a short source is 


$$
\frac{d^{4} N(0,0,0,0)}{d x^{\prime} d x d z^{\prime} d z}=1.256 \times 10^{8} \gamma f G_{1}\left(\frac{1}{\sqrt{2 \pi} \sigma_{x}}\right) \cdot\left(\frac{1}{2 \pi \sigma_{z} \sqrt{\sigma_{z}{ }^{2}+\sigma_{R}^{2}}}\right),
$$

or alternatively

$$
=3.46 \times 10^{4} \frac{r^{2} \mathrm{H}_{2}}{2 \pi \sigma_{\mathrm{x} 2} \sigma_{2}} \text { if } \sigma_{\mathrm{R}} \gg \sigma_{\mathrm{z}^{\prime}}
$$

photons per sec, $\operatorname{mm}^{2}, \operatorname{mrad}^{2}$ for $1 \%$ band width, 1 milliamp current, 1 milliradian of orbit, with $\sigma_{x}$ and $\sigma_{z}$ in $\mathrm{mm} . \mathrm{Eq} .(232 \mathrm{~b})$ can be immediately derived by multiplying Eq. (221) by the probability dxdi/2 $\pi \sigma_{\mathbf{x}} \sigma_{2}{ }^{\cdot}$ Note that the central brightness is not proportional simply to the total flux divided by $\left(\sigma_{X} \sigma_{X}, \sigma_{z} \sigma_{Z}\right)$; this is due, of course, to the extended nature of the source. A "bright" source is designed so that $\sigma_{z},<\sigma_{R}$ and, in this case, $\sigma_{z}$, may be dropped in Eq. (232a). With $\sigma_{R} \curvearrowright \gamma^{-1},(232)$ indicates that the central brightness is proportional to $\gamma^{2} / \sigma_{z} \sigma_{x}$. It is clear that brightness is increased with increase of $\gamma$. However, the gain is less pronounced than $\gamma^{2}$ for wavelengths greater than $\lambda_{c}$ due to the fact that, with the shift of the characteristic spectrum (Fig. 2) to shorter wavelengths, at any given wavelength one is progressively at a lower point of the intensity distribution. With the advantages of large $\gamma$ come a few negative factors also, not all of which are monetary: higher power densities on first optical elements and, because large $\gamma$ generally requires large: bending radil, longer distance between source point and first optics.

The results above were derived for a source segment whose center is a waist or focus of the electron beam such that the axes of its ellipses are the phase space coordinate axes. While such waists do occur (Fig. 19), in practice they rarely coincide with actual source centers. If the source center is described by a tilted ellipse, it is 
often useful to find some nearby point along the orbit at which the ellipse Is vertical and derive the effective extended source with respect to it. The derivation is more tedious than what is considered above. This means that actuä sources w11 rarely be accurately symmetric in phase space. Green concluded, however, that Eqs. (226 and 231) for the central fluxes and Eqs.(232) 'for the central brightness are valid approximations applicable to the situation in which $\sigma_{z^{\prime}}<\sigma_{R}$ and in which the source is "short", meaning $s \sigma_{R}<\sigma_{z}$.

In the foregoing, no consideration has been given to the fact the distribution of electrons in $x$ is affected by the momentum dispersion; that is, the electron ellipses have been calculated on the basis that all electrons have the ideal orbit momentum $p_{0}$. Actually, there is a distribution of momenta around $p_{0}$, and, in accord with Eq. 73, the radial beam size is

$$
\left\{\varepsilon_{x} \beta_{x}(s)+\left[n(s) \frac{\Delta p}{p_{0}}\right]^{2}\right\}^{\frac{1}{2}}
$$

Here $\varepsilon_{x}, B_{X}(s)$ and $n(s)$ are the emittance, amplitude function and dispersion function respectively. Now $n$, because it varies around the orbit, introduces a correspondingly varying contribution to the horizontal source size. This variation is not rapid and has but small effects. 


\subsection{Interaction of the Source with Optical Elements}

We have just seen that the phase space distribution of the photon source, although it is relatively broad in both horizontal dimensions, is narrow and centered at $z=z^{\prime}=0$ in the vertical. As a consequence, most optical systems intended for use with synchrotron radiation beams are designed with horizontal slits for vertical deflection. This geometry, advantageous with respact to resolution and intensity, has the important additional advantage that vertical deflection is more efficlent than horizontal for radiation which is predominantly of horizontal polarization, E in the electron orbit plane. With this general geometry we need to consider the fact that a horizontal instrument aperture, such as the finite horizontal length along a slit narrow vertically, acts to limit horizontal phase space. If, at a point $D=0$ corresponaing to the center of the source segment, we have the source phase space properties shown at the left In Fig. 24, then, through an aperture at distance $D_{1}$ from the segment center, the effective source corresponds to the sketch on the right. A transformation of the aperture back to $D=0$ shows that the aperture imposes limits there, as indicated by the two diagonal lines which cut the left sketch.

There is an important systematic varfation of flux in $x^{\prime}$, the horizontal angle, across the aperture. This is associated with the progression, from point to point in s along the orbit, of the "center" of the source segment element corresponding to and effective at each point along the aperture length. In contrast to the changing $x^{\prime}$ flux distribution along the aperture, the flux distribution in $z^{*}$ is constant 

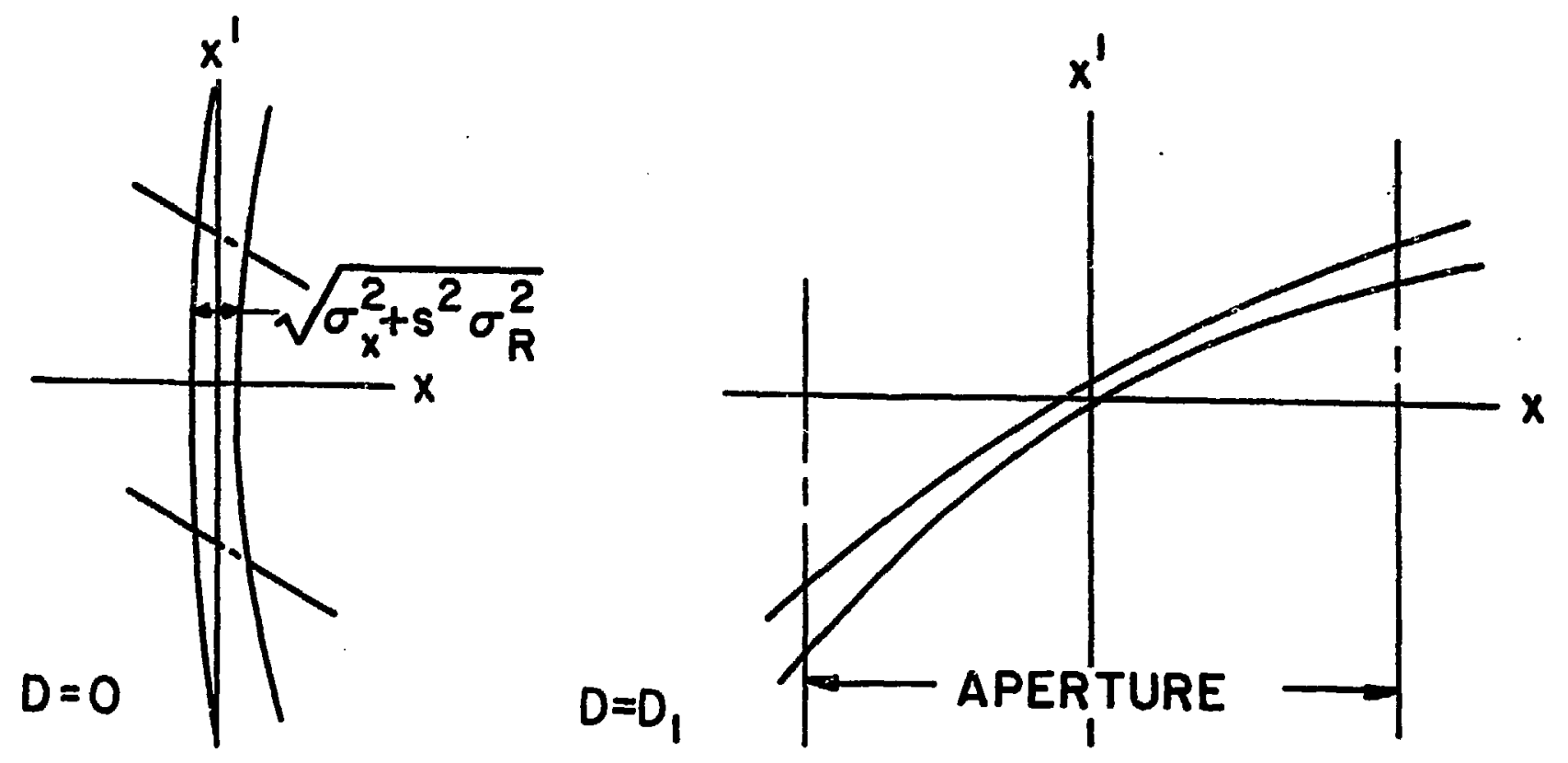
because, as can be seen from Fig. 22, the $z^{\prime}$ distribution does not vary appreclably among the segment elements making up the source. The flux distribution along the linear dimension $z$ does vary along the aperture. This can be shown by integration of the $z, z^{\prime}$ phase space over $z^{\prime}$; for an orbit segment ds located at $s$, we see, following Green (1976), that

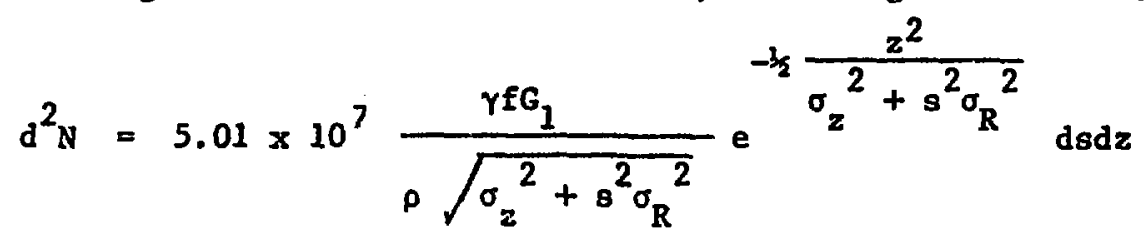

photons/sec, $1 \%$ bandwidth, and 1 milliampere electron current. Here, $p$ is in meters, $s, z$ and $\sigma_{z}$ in militmeters. Note that it is the correspondence of individual points along the aperture with "specific" $s$ values that causes the variation in $\mathrm{d}^{2} \mathrm{~N}$. For a storage $x i n g$ of say $3 / 4 \mathrm{GeV}$, $\sigma_{R}$ at $\lambda_{C}$ is about $1 / 2$ milliradian, and if the lattice is designed for high brightness $\sigma_{2} \sim 0.1$ millimeter. A large angle port subtending 75-90 milliradians of orbit intercepts a segment of about $20 \mathrm{~cm}$, equivalent to $\mathrm{s} \approx 10 \mathrm{~cm}$. In such a case $s \sigma_{\mathrm{R}} / \sigma_{\mathrm{z}} \sim 1 / 2$ and the variation in central intensity $(\mathrm{z}=0)$ across the port is then roughly $10 \%$. At longer wavelengths $\sigma_{R}$ is larger and this intensity effect is more pronounced. For a ring of larger radius, with higher electron energy and with $\sigma_{z}$ the same, $s$ will ordinarily be somewhat smaller and $\sigma_{R}\left(\lambda_{c}\right)$ considerably smalier, so that the intensity variation is less. 
Consider now a slit of width 2d,with its length on the horizontal, which Intercepts vertical phase space at $D_{1}$, as sketched in FIg. 25 on the right. Suppose there to be a source, at $D=0$, described by the contour

$$
\frac{\sigma_{z^{\prime}}}{\sigma_{z}} z^{2}+\frac{\sigma_{z}}{\sigma_{z^{\prime}}} z^{\prime 2}=\sigma_{z^{\prime}} \sigma_{z^{\prime}}
$$

where $\sigma_{z}$ and $\sigma_{Z}$, are parameters of the photon source segment. The emittance, that 1s, the ellipse area or phase space divided by $\pi$, is $\sigma_{2} \sigma_{2} \cdot$ At $D=D_{1}$, the emittance is preserved but the contour of the photon beam becomes

$$
\frac{\sigma_{Z^{\prime}}}{\sigma_{z}} z^{2}-\frac{2 \sigma_{Z^{\prime} D_{1}}}{\sigma_{z}} z^{\prime}+\left[\frac{\sigma_{z}}{\sigma_{z^{\prime}}}+\frac{\sigma_{z^{\prime} D_{1}^{2}}}{\sigma_{z}}\right] z^{\prime 2}=\sigma_{z^{\prime} z^{\prime}} \cdot
$$

The maxima are no longer the intercepts, as is obvious from the figure. The $Z^{\prime}$ intercept is

$$
z^{\prime}=\frac{ \pm \sigma_{z}}{\sqrt{\left(\sigma_{z} / \sigma_{z^{\prime}}\right)^{2}+D_{1}^{2}}}
$$

In the limit, as the slit width goes to zero, this intercept is the : maximum angular resolution (minimum accepted angle) attainable. In general $D_{1}>\sigma_{2} / \sigma_{2}$, , and this angle is then $\sigma_{2} / D_{1}$. For a finite but small slit opening $2 d$, the half angle is approximately

$$
z^{\prime}(d) \simeq z^{\prime}(d=0)+\frac{d z^{\prime}}{d z} d,
$$



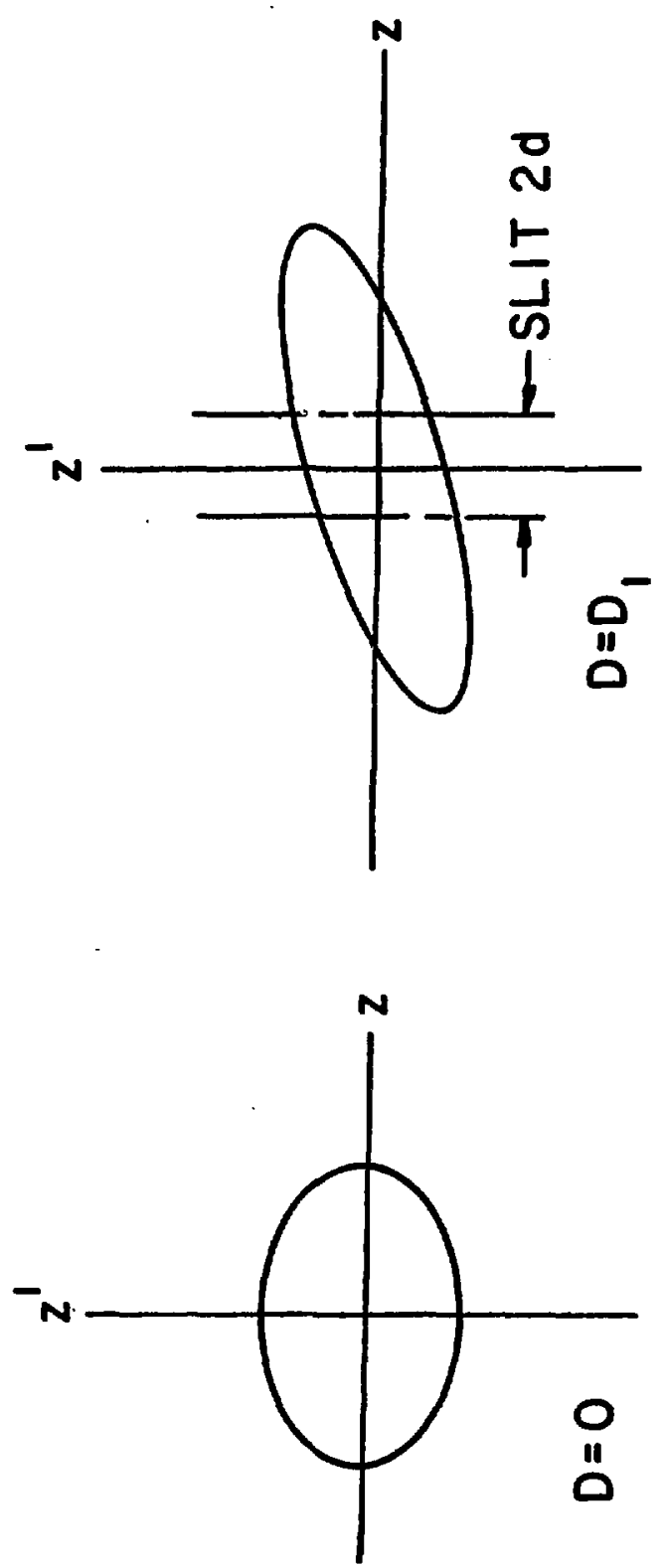
and, obtaining the derivative from (235), we find

$$
\begin{aligned}
z^{\prime}(1) & \simeq \frac{\sigma_{z}}{\sqrt{\left(\sigma_{z} / \sigma_{z^{\prime}}\right)^{2}+D_{1}^{2}}}+\frac{D_{1} d}{\left(\sigma_{z} / \sigma_{z^{\prime}}\right)^{2}+D_{1}{ }^{2}} \\
& \simeq \frac{\sigma_{z}+d}{D_{1}}
\end{aligned}
$$

One may easily make a rough estimate of the fraction of the flux passing through the slit. The area of the ellipse intercepted by the slit is approximately a parallelogram of area

$$
4 z^{\prime}(d=0) d=\frac{4 d \sigma_{z} \sigma_{z^{\prime}}}{\sqrt{\sigma_{z}{ }^{2}+\sigma_{z}{ }^{2} D_{1}^{2}}}
$$

Since the area of the ellipse is $\pi z^{\sigma} z^{\prime}$, the fraction of $z, z^{\prime}$ flux transmitted through the slit becomes

$$
\frac{4 d}{\pi \sqrt{\sigma_{z}{ }^{2}+\sigma_{z},{ }^{2} D_{1}{ }^{2}}}
$$

A two crystal monochromator, in the antiparallel configuration, is unusual in that it constitutes an angle defining slit in phase space, as indicated in Fig. 26. We assume vertical dispersion and a crystal, located at $D=D_{1}$, of perfection such that the angular acceptance, $\Delta Z^{\prime}$, is of the order ten seconds of arc. Barring focussing elements between source and the monocinromator, this angle transforms back to the source 

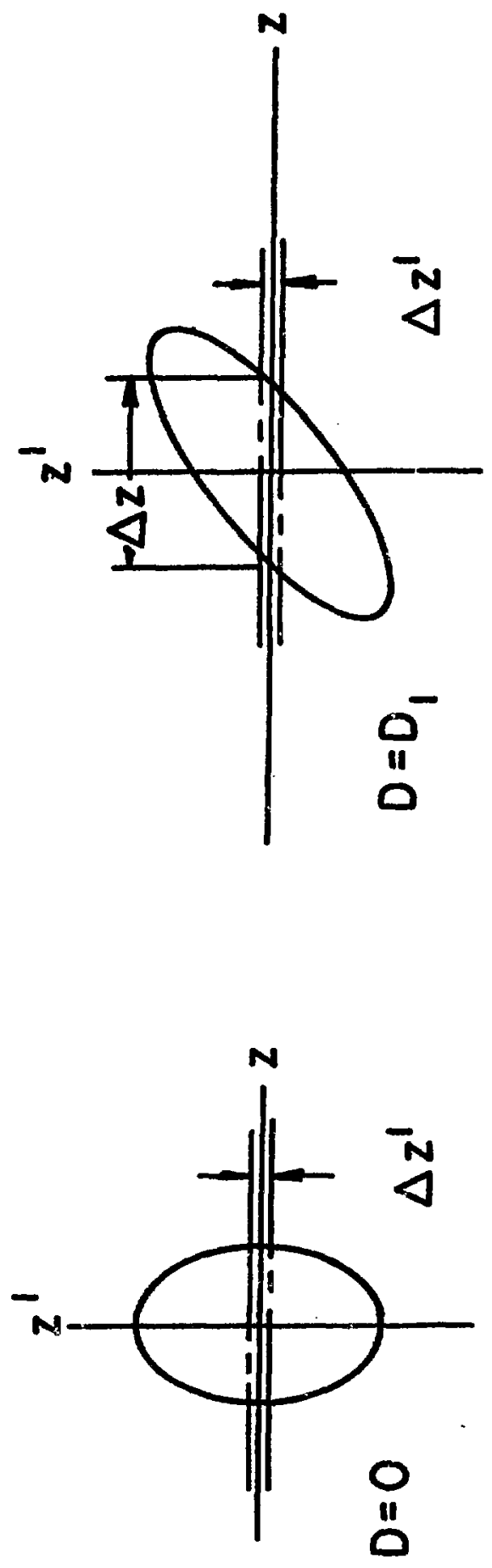
unchanged. The angular flux is [Green (1976)]

$$
\operatorname{dN}\left(Z^{\prime}\right) i 7 \times 10^{7} \gamma^{2} H_{2} \frac{W}{D_{1}} \frac{\sigma_{R}}{\sqrt{\sigma_{Z^{\prime}}{ }^{2}+\sigma_{R}^{2}}} \Delta Z^{\prime} \text { photons/sec, 1\%bandwidth, } 1 \text { milliamp }
$$

where $W$, the crystal width, and $D_{1}$ are in comon units and $\Delta Z^{\prime}$ is in milliradians. The horizontal angle accepted by the monochromator $1 \mathrm{w} / \mathrm{D}_{1}$ and $i t$ corresponds to the angle, $s / \rho$, subtended by the source segment.

Photon beams at visible, UV and even X-ray wavelengths may be focused in the horizontal, the vertical, or in both dimensions simultaneously. However, any change in transverse linear dimension, $x$ or $z$, is necessarily accompanied by a change of opposite sense in angle $x^{\prime}$ or $z^{\prime}$; in other words enfttance and brightness are conserved. For experiments which require high resolution both in angle and in linear dimension there is thus no substitute for....high source brightness. 


\section{SYNCHROTRON RADIATION: THEORY VERSUS EXPERIMENT}

A detalled comparison between the theoretical description of characteristics of synchrotron Iight (SectionII) and experimental observations is not easily made because it is quite difficult to make absolute measurements of some of the important parameters with the desired accuracy. For example, measurements of the radiation spectrum suffer from the fact that different methods must be used for different wavelength regions and absolute sengitivity varies with the technique and is often wavelength dependent for a given technique. Even some of the geometrical measurements of orbit characteristics, such as electron bunch cross section and deviation of the orbit from circular symmetry, are not easily determined.

Shortly after the characteristic light was observed for the first time at the 70-MeV synchrotron of General Electric Company, Elder and coworkers (1947 and 1948) Investigated the spectral distribution at visible wavelerigths. Later, in their classic paper, Tomboulian and Hartman (1956) reported studies of the spectrum as far down as $60 \AA$ and of angular distributions as we11, which they had made with the 300-MeV synchrotron at Corne11. These studies were difficult because they were carried out, by necessity at. synchrotrons rather than at storage rings. For each observation, data were collected over a number of acceleration cycles, so that in addition to the fact that each observation was made over a range of electron energies, there was undoubtedly cycle-to-cycle drift and fluctuation. Also, these investlgators had to rely on intensity measurements made with photographic emulsions over the range of wavelengths. Calibration of sensitivity over a range of wavelengths is even now a troublesome matter. 
Within the accuracy of these older measurements it was found that the results are in accord with theory, and this situation has held as subsequent investigations have reduced the uncertainties. While the later investigations are certainly importani, it seems fair to say that Tomboulian and Hartman (1956) provided experimental verification for the classical relativistic theory of synchrotron radiation.

Joos (1960), also at Corne11, was the first to observe the polarization intensity distribution as a function of angle. These measurements have been made with greater precision by Codling and Madden (1965), whose results appear in FIg. 27. Bathow and coworkers (1966) complemented the long wavelength work by studying the total flux, polarization, and angular distribution of hard $x \rightarrow-$ anating from 4- to 6-GeV electrons in the synchrotron DESY at Hami $4 \mathrm{~g}$. Their flux measurements for $\lambda<\lambda_{\mathrm{c}}$, shown In Fig. 28, are indicative of the quality of accord they obtained between their observations and theory.

In the course of describing their results, Tomboulian and Hartman (1956) pointed out that, because theory accounted very well for the spectra they observed, synchrotron light sources show promise as standards for callbration of optical instruments and of other sources. The National Bureau of Stand-rds has been concerned with work of this kind and operates SURF II, a 240-MeV storage ring. In SURF II the orbit lies between the poles of a single large magnet. Therefore, to the extent that $\vec{H}$ is circunferentially constant, the electron orbit is circular, an advantage for absolute intensity work. Ederex and coworkers (1975) analyzed the exrors introduced in their experiments by the uncertainties 


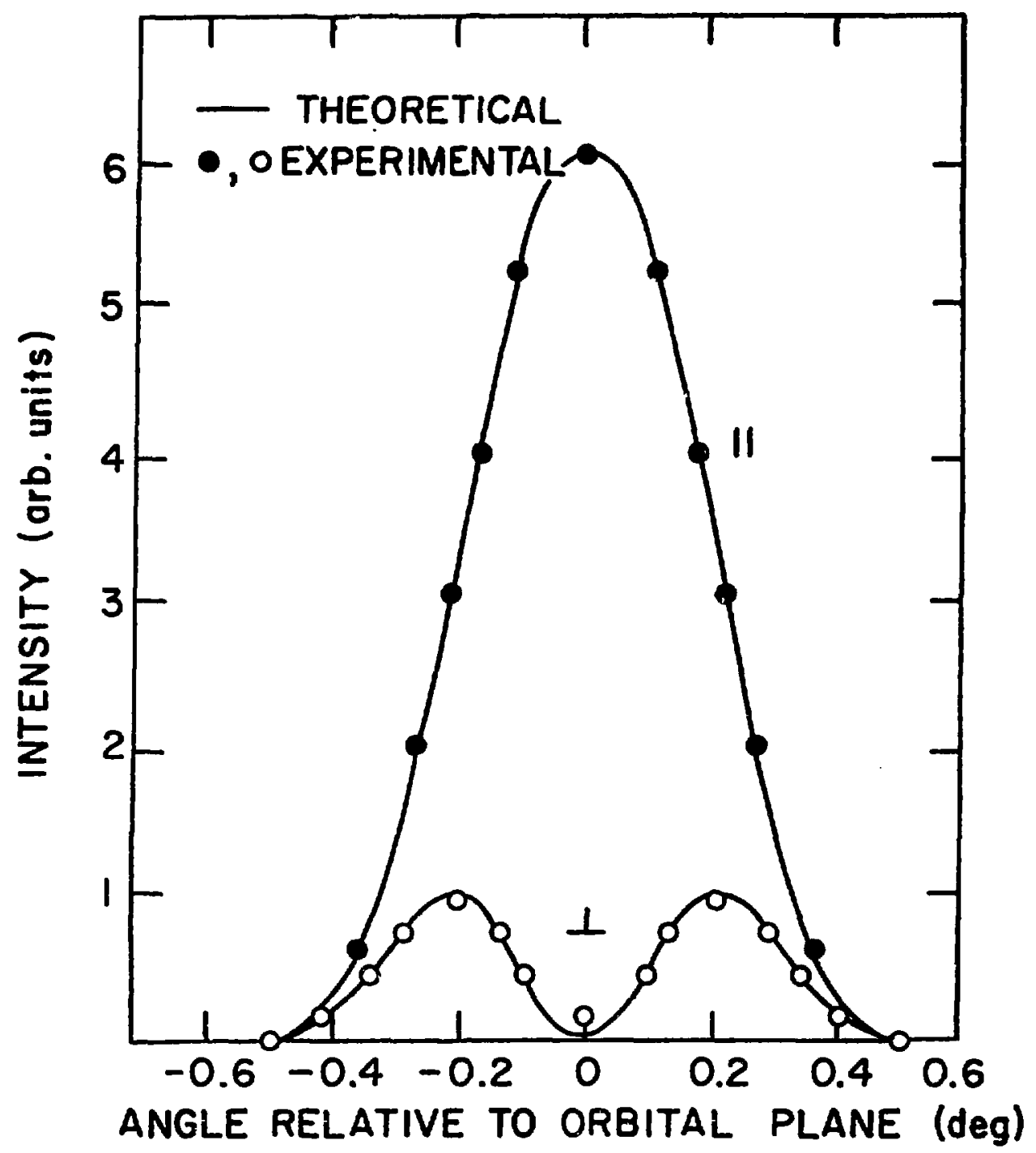

Fig27 
PHOTON FLUX (PHOTONS / eV·sec·ELECTRON)

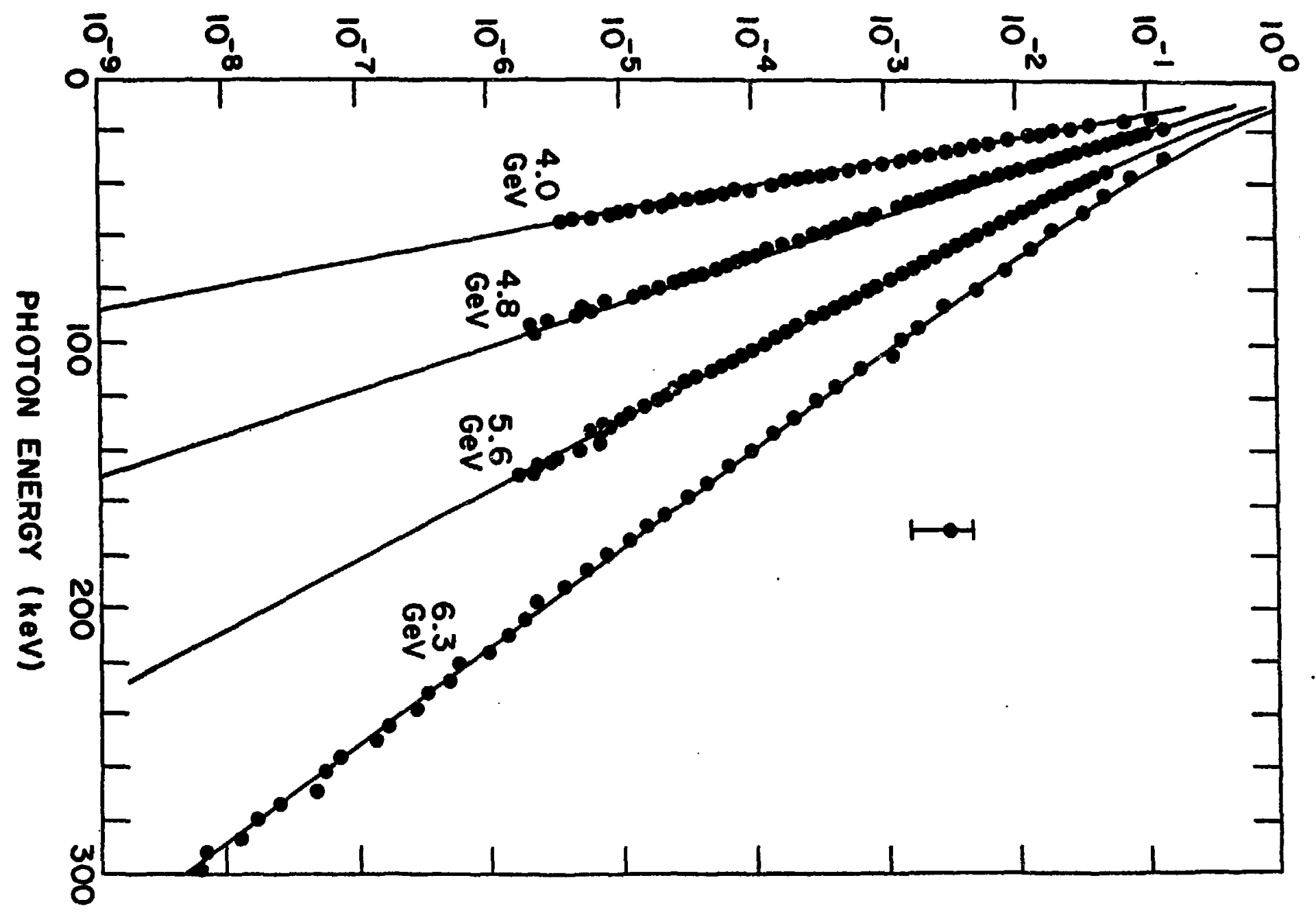


In electron energy, current and orbit position; they concluded that there was $3 \%$ random error and $6 \%$ systematic error in use of SURF II as a VWV standard. A major source of error was in determination of the number of electrons in the beam. Currently, the group is in the process of calibrating photon detector response by observing the light from a single electron. With improvements in technique and instrumentation these workers anticipate achieving in 1980 an accuracy of better than $1 \%$. The long range accuracy target is $0.1 \%$-presuming, of course, that theory and experiment maintain their agreenent. 


\section{WIGGLERS, UNDULATORS AND THE FREE ELECTRON LASER}

\subsection{Introduction}

The discussion of this chapter has concentrated, thus far, on the radiation emanating from the bending magnets of an accelerator or storage ring. We shall now consider the possibility of obtaining a different kind of radiation source by placing in a straight section of a machine, such as Aladdin sketched in Fig. 8, a magnetic device whtch wiggles the electrons. A device of this type, indicated in Fig. 29, In general consists of $h$ periods of length $\lambda_{0}$, the overall length being

$$
\chi=h \lambda_{0}
$$

If the wiggler is comprised of one or a few perlods, the radiated spectrum is essentially that of "normal" synchrotron radiation, characterized by the crittcal wavelength $\lambda_{c}[E q .(8)]$ corresponding to the wiggler's magnetic fleld strength. By the use of superconducting colls, magnetic fields significantly stronger $(\approx 40 \sim 50$ kilogauss) than those in bending magnets ( $\approx 10$ kilogauss) can be obtained. The central brightness of the wiggler source can be optimized by placing. it in a low- $\beta$ insertion (Fig. 14), where the horizontal and vertical electron beam sizes are smaller than in the bending magnets. Also, as discussed in Section 3.14, if the insertions are designed so that the dispersion $n$ is zero in them, the high magnetic flelds of the wigglers will reduce rather than increase the emittance of the electron beam.

In a wiggler having several periods, the orbit geometry is different from that in a bending magnet, there being in the multipole wiggler several source segments on a line. This clearly allows for a flux enhancement, but under certain circumstances this benefit may be 


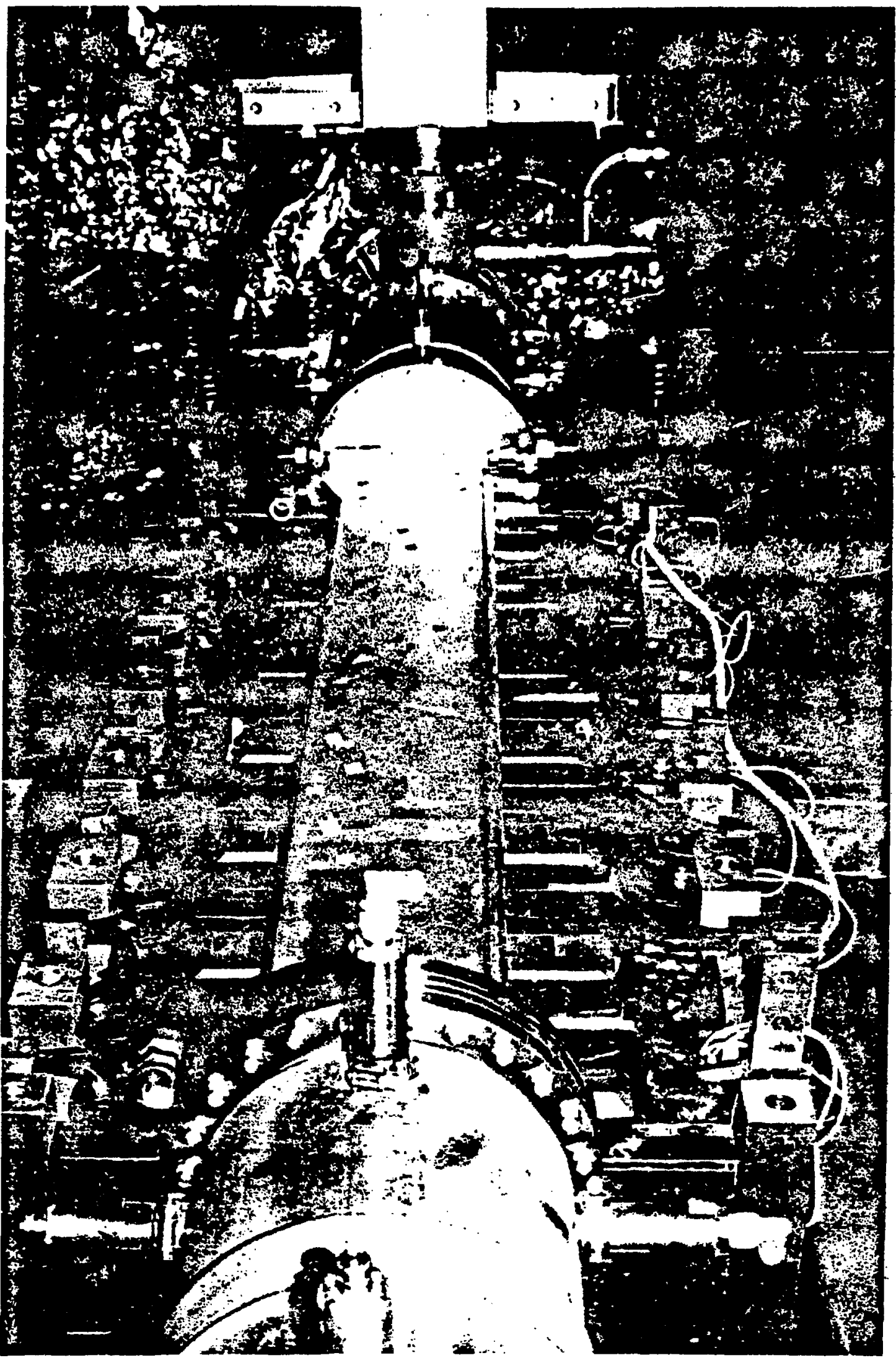

Fig 29 
reduced by an increase in the size of the effective source. In Section 6.3 we apply, to the wiggler, the type of phase space analysis used in Section 4 to discuss the bending magnet source.

When a wiggler magnet has many perlods, the radiated spectrum is no longer that of normal synchrotron radiation. Provided the magnetic fleld is not too great, the spectrum is sharply peaked about the wavelength

$$
\lambda=\lambda_{0} / 2 \gamma^{2}
$$

We see that the characteristic wavelength at a given electron energy is determined not by the magnetic field strength, as in the case of normal synchrotron radiation, but by the magnetic spatial perlodicity. We shall use the term "undulator" to refer to a multiperiod wiggler magnet of this type,designed to produce narrow-band radiation. Such a device is also often referred to as a "coherent wiggler". The history of the undulator can be traced back to. the aarly 1950's when Motz (1951) first analyzed and later demonstrated [Motz, Thon, and Whitehusst (1953)] the operation of such a device for the generation of millimeter wavelength and visible radiation. Today there is great interest in undulators as sources of intense narrow-band radiation in the ultra-violet and soft x-ray regions, see e.g. Alferov, Bashmakov and Bessonov (1973) and Kincald (1977). We discuss the spectrum of undulator radiation in Sections 6.4 and 6.5 .

As is seen from Eq. (242), shorter radiated wavelengths result from reducing the undulator wavelength $\lambda_{0}$. There $1 s$, however, a practical 1imit. As a crude rule of thumb, In order to obtain substantial magnetic field strength at the middle of each half-peziod, $\lambda_{0}$ must be roughly 1.5 to 2 times the gap between magnet pole faces. On the other hand, electron orbits undergo large excursions at injection, and vacuum 
chamber cross-sections of at least several centimeters are required. As a result, $\lambda_{0}$ of less than $4 \mathrm{~cm}$ is very difficult to achieve, though not impossible if the mechanical design allows movement of the magnet into position after injection.

A source of much recent excitement is the fact that an electron beam passing through the static magnetic fleld of an undulator can amplify radiation moving with the electrons through the field. This seems first to have been realized by Motz and Nakamura (1959, 1960), who showed that an undulator can be used as an amplifier of mfllimeter waves in a rectangular waveguide. Several devices of this type, called ubitrons (undulated beam interaction), were built by PhIllips (1960). In the ubitron, the basic gain process is dominated by collective effects, i.e. many electrons oscillating together to yield amplification non-linear in the current. The free electron laser (FEL), as recently developed at Stanford University, was originally proposed and analyzed by Madey (1971). This conslats of an undulator situated in an optical cavity, and, in contrast to the ubitron, its gain mecharism does not depend upon collective effects. A report of actual opticàl gain at a wavelength of $10.6 \mu \mathrm{m}$ was made by Ellas et al. (1976), and the successful operation of a FEL oscillator at a wavelength of $3.4 \mu \mathrm{m}$ was reported about one year later by Deacon et al. (1977). In Section 6.6 we give a brief discussion of the basic gain mechanism, following the approach of Colson (1978), who showed that the Stanford FEL can be described by a theory based upon the one-body classical Lorentz force equations for the static periodic magnetic tield of an undulator and a plane electromagnetic wave.

\subsection{A Single Period Wavelength Shifter}

Consider a single period wiggler with the magnetic fleld and orbit 
sketched in Fig. 30. The entering and exiting orbit segments are in line so that a storage ring could operate with the magnet elther excited or not. In general, one would employ superconducting magnets at fields of 40 to 50 kilogauss to produce a spectrum whose critical wavelength would be typically one-fifth that from a bending magnet. One can obtaln hard radiation relar tively economically by providing a few ports from short orbit segments at high magnetic field on a ring operating at moderate electron energies. Accomplishing this with wavelength shifters placed in straight sections, rather than with a set of short high field bending magnets, has the advantage of decoupling the use of somewhat complex superconducting magnets from normal machine operation. Also, as was seen in Section 3.14, the process of photon emission makes the principal contribution to finite electron beam size and thus limits source brightness. The effect is the more severe, the larger the dispersion function $\eta$ in the region of emission. In a lattice such as that represented In Fig. 14 the electron optics are designed so that the dispersion function has zero value in the Insertion region. Th1s eliminates the adverse effect on beam size of generating hard radiation from a wiggler placed in the insertion.

In general, one will view such a wiggler parallel to the axis of the unperturbed orbit in the straight section. The photon port, through the vacuum wall, is placed just at the adjacent down stream bending magnet. Observation at other angles is generally difficult because of the presence of such obstructions as quadrupole magnets which encircle the racuun enclosure and make take off angles of $5^{\circ}$ or $10^{\circ}$ "necessary. Inspection of Fig. 30 shows th th the field strength is small where the orbit has 1ts largest off axis angles. If the orbit is to be inmaximum field at some given angle $\theta_{m}$, the angular excursion required will be nearly twice $\theta_{m}$ and the total 
$X$, ORBIT DISPLACEMENT
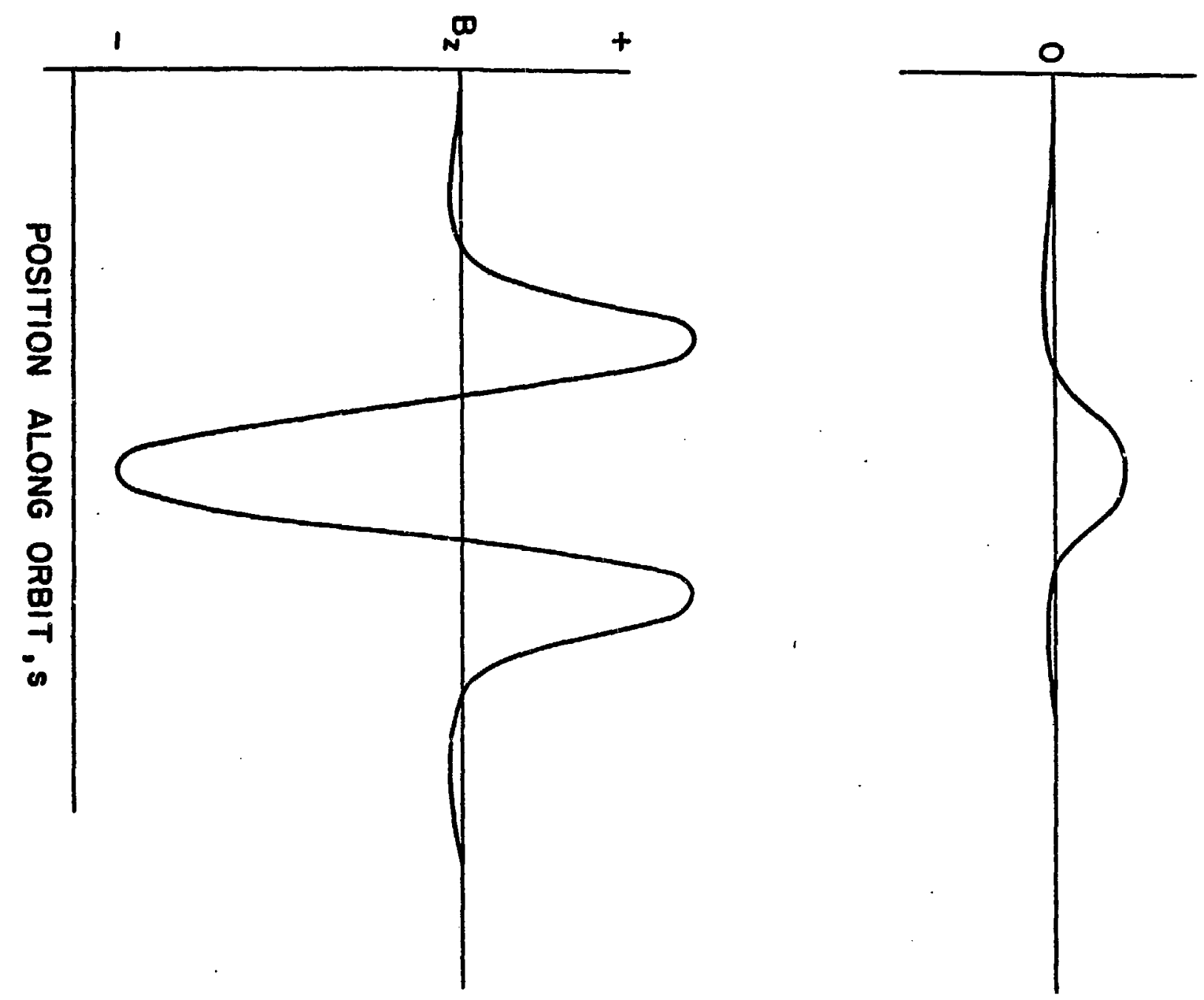
orbital angle executed must be roughly six to elght times $\theta_{m}$. This is costly in synchrotron radiation power which must be supplied by the RF system only to be dissipated in heating the vacuum chamber wa11s.

There are disadvantages in utllizing a wavelength shifter beam at zero degrees. The optical element nearest the source is necessarily farther from it than is the case if the source is in a bending magnet. Again, the angular cone of radiation which may be extracted, defined by the cross-sectional dimensions of the vacuum chamber, Is strongly limited. Depending on partlcular dimensions in the storage ring, 10 to 20 milliradians is the maximum extractable on-axis horizontal angle of radiation from a single or multiperiod wiggler situated in a low- $\beta$ insertion.

Like the radiation from a bending magnet, the $E$ vector of the radiation from a transverse wiggler of the type shown in Fig. 29 . is polarized strongly in the horizontal plane. Now, crystals and gratings reflect most efficiently when $E$ lies in the plane of their surfaces; therefore, efficient scattering of normal synchrotron light is in the vertical plane. In many cases vertical scattering is advantageous also because the vertical source size is smaller than the horizontal. For some experiments, however, it may be useful to scatter efficiently in the horizontal. The $\underline{E}$ vector is of course vertical if the magnetic field direction is horizontal, and horizontal field wigglers may become useful.

\subsection{Wigglers with Several Periods}

Consider an $n$ pole wiggler in which $n$ is not so large as qualitatively to change the spectrum. Such a source 
is made up of $n$ segmients of orbit

in a Iine and,as a result, it is not the curved extended ribbon sketched in Fig. 23. Instead, the effective source assoclated with either end of the wiggler, when transformed to the middle, is an ellipse centered at $x=x^{\prime}=0$, as was seen for the vertical coordinates in Fig. 22.

Vertical source characteristics in this case are essentially those already determined for a bending magnet. The angular spread of the radiation from the center of the wiggler is

$$
\sigma_{z^{\prime}}=\left(\sigma_{z^{\prime}}^{2}+\sigma_{R}^{2}\right)^{\frac{1}{2}}
$$

It is clearly undesirable for $\sigma_{z}$, to be much larger than the natural radiation width, therefore we require

$$
\sigma_{2} \lesssim \sigma_{R}
$$

A photon emitted from one end of the wiggler, $s \equiv \mathcal{X} / 2$, at position $z_{0}$ and angle $z_{0}^{1}$ appears as though it originated at the center from $\left(z_{0}-\frac{\mathcal{L} z_{0}^{\prime}}{2}, z_{0}^{\prime}\right)$, and the effective size of the synthesized source is

$$
\sigma_{z}=\left[\sigma_{z}^{2}+\left(\frac{x}{2}\right)^{2} \sigma_{R}^{2}\right]^{\frac{1}{2}}
$$

In order that $\sigma_{z}$ be not much larger than $\sigma_{z}$, we impose the condition

$$
\frac{\chi \sigma_{R}}{2} \leq \sigma_{2}
$$

When the properties of the phase ellipse (Section 3.5), are taken into 
account, the inequalities (244) and (246) imply that

$$
\frac{\mathscr{Z}}{2} \leq \beta_{\mathrm{z}} .
$$

If these inequalities are satisfied, the central vertical phase space density increases linearly with the number of wiggler periods, i.e.

$$
\frac{d^{2} N}{d z^{\prime} z^{\prime}}\left(z=z^{\prime}=0\right) \sim \frac{n}{\sigma_{R} \sigma_{z}} .
$$

For 2 large enough to violate (246) the dens. $-y$ increase is less than linear, and it eventually becomes logarithmic in $n$.

In the horizontal plane, the angular distribution of the radiation is determined predominantly by the geometry of the electron orbit. For an observer at angle $\theta_{0}$ with respect to the wiggler axis, the effective size of the synthesized source is

$$
\sigma_{x}=\left[\sigma_{x}^{2}+\left(\theta_{0}^{2}+\sigma_{R}^{2}\right)\left(\frac{x}{2}\right)^{2}\right]^{\frac{1}{2}} .
$$

In the forward direction, the analysis is the same as for vertical phase space; i.e., when

$$
\sigma_{\mathbf{x}^{\prime}} \leqslant \sigma_{\mathrm{R}}
$$

and

$$
\frac{\mathcal{L}\left(\theta_{0}+\sigma_{R}\right)}{2} \leqslant \sigma_{x},
$$

then

$$
\frac{d^{2} N}{d x d x^{\prime}}\left(x=x^{\prime}=0\right) \sim \frac{n}{\sigma_{R} \sigma_{x}},
$$


and the central brightness is

$$
\frac{d^{4} N}{d X d Z^{\prime} d z d Z^{\prime}}\left(X=X^{\prime}=Z=Z^{\prime}=0\right) \sim \frac{n}{\sigma_{R}^{2} \sigma^{\sigma}{ }^{\prime}} \text { : }
$$

As the number of wiggler periods is increased, the length $\mathcal{X}$ eventually becomes sufficientiy large to violate condition (251). Although further Increase in $\chi$ results in a linear Increase in the number of photons, the $\mathcal{Z}$ increase causes a linear increase in source size. The brightness thus tends to saturate and increases on ly logarithmically with $\boldsymbol{\eta}$. At large viewing angles, $\theta_{0}>\sigma_{x} / \lambda_{0}$, there is essentially no increase in brightness with increase in $n$ since (251) is violated. Now, from Section 4 we saw that $\sigma_{x}$ is typically $1 / 2$ to $1 \mathrm{~mm}$ in a bright source; for typical $\lambda_{0}$ of 5 to $10 \mathrm{~cm}$ we then have

$$
5<\frac{\sigma_{x}}{\lambda_{0}} \leqslant 20 \mathrm{mrad}
$$

As was noted in the preceding subsections, the opening half-angle of radiation which may be extracted in the forward direction is determined by the size of the machine vacuum chamber and is typically 10 to $20 \mathrm{mrad}$. In such a case $\theta_{0} \sim \sigma_{x} / \lambda_{0}$ and Eqs. (252) and (253) hold, subject to the Inequalities (244), (246), (250) and (251). A port, sufficiently off the wiggler axis to avold mechanical obstructions, will have a source brightness independent of wiggler length.

6.4 Emission from an Undulator-Transverse Magnetic Field

A transverse undulator is designed so that the wagnetic field in the midplane is in the vertical direction and, to a good approximation, has the sinusoidal dependence, 


$$
B_{z}=B_{0} \sin \left(2 \pi \zeta / \lambda_{0}\right)
$$

Here $\lambda_{0}$ is the undulator wavelength and $\zeta$ is the coordinate along the axis of the magnet. The equations of motion of an electron in this field are given by

$$
\operatorname{rm} \ddot{\zeta}=e \frac{\dot{x}}{c} B_{0} \sin \left(2 \pi \zeta / \lambda_{0}\right)
$$

and

$$
r_{\operatorname{mix}}=-\mathrm{e} \frac{\dot{\xi}}{\mathrm{c}} \mathrm{B}_{0} \sin \left(2 \pi \zeta / \lambda_{0}\right)
$$

For convenience, we define the parameters $\delta_{w}$ and $k$, by

$$
\delta_{w}=\frac{K}{\gamma}=\frac{e}{\gamma \operatorname{rc}^{2} B} \frac{\lambda_{o} B_{0}}{2 \pi},
$$

where $\beta$ is, of course, $v / c$ for the electron; $\delta_{w}$ can also be written in terms of the bending radius $\rho_{0}$ corresponding to the peak field $B_{0}$ as

$$
\delta_{w} \equiv \frac{\lambda_{0}}{2 \pi \rho_{0}} .
$$

It follows from Eqs. $(255 a, b)$ that

$$
\dot{x}=\beta c \delta_{w} \cos \left(2 \pi \zeta / \lambda_{0}\right)
$$

and

$$
\dot{\zeta}^{2}=\beta^{2} c^{2}-\dot{x}^{2}
$$

Then

$$
\dot{x}=\left(\beta c \delta_{w}\right) \text { sn }\left(\Omega_{w} t ; \delta_{w}\right) \text {, }
$$

whexe $\Omega_{w} \equiv 2 \pi \beta c / \lambda_{0} \approx 2 \pi c / \lambda_{0}$ is the wiggle frequency imposed on an electron 
as it tranverses the wiggler and $8 n(u ; k)$ is the elliptic function of modulus $k$.

With (256a) we may arrive at

$$
\delta_{w} r=k \cong \frac{B_{0}(k i l o g a u s s) \lambda_{0}(\mathrm{~cm})}{10}
$$

For a highly relativistic electron, $Y$ is very large, so that for reasonable values of $B_{o}$ and $\lambda_{o}, \delta_{w}$ is small. In this case Eqs. $(257 a, b)$ simplify, yielding

$$
\dot{\zeta} \cong \beta c\left[1-\frac{1}{2} \quad \delta_{w}^{2} \cos ^{2}\left(2 \pi \zeta / \lambda_{0}\right)\right] ;
$$

hence the average velocity along the undulator axis is

$$
<\dot{\zeta}>\approx \mathrm{BC}\left(1-\frac{\delta^{2}}{4}\right) \equiv \beta *^{*} \mathrm{C} .
$$

The electron trajectory $\underline{I}(t)=[x(t), z(t), \zeta(t)]$ has the approximate form

$$
\underline{r}(t)=\left(\frac{\beta c \delta_{w}}{\Omega_{w}} \sin \Omega_{w} t, 0, \beta^{*} c t-\frac{\beta c \delta_{w}^{2}}{8 \Omega_{w}} \sin 2 \Omega_{w} t\right) .
$$

In the x-coordinate, the amplitude of oscillation is

$$
a=\frac{\beta c \delta_{w}}{\Omega_{w}}=\rho_{0}^{-1}\left(\frac{\lambda_{0}}{2 \pi}\right)^{2}
$$

with $\rho_{0}$ the bending radius corresponding to the field $B_{0}$. The maximum angular deviation of the electron frow the undulator axis is equal to $\delta_{w}$. The basic properties of the undulator radiation spectrum can be understood intuitively by following Motz (1951) and Purcell (1972) in using a Lorentz transformation to a frame $\tilde{F}$ which keeps up, on average, 
with an electron passing through the magnet. Relative to the lab frane $F$, the frame $\widetilde{F}$ moves at the average velocity $B * c$, defined in Eq. (261). The relativistic parameter $\gamma^{*}=\left(1-\beta *^{2}\right)^{-\frac{1}{2}}$ is approximately given by

$$
r * \tau\left(1+R^{2} / 2\right]^{-\alpha}
$$

Under the Lorentz transformation to $\widetilde{F}$, Eq. (262) gives the electron trajectory

$$
\tilde{x}=\frac{\beta c \delta_{w}}{\Omega_{w}} \sin \left[\Omega_{w} \hat{\gamma}^{*}(\tilde{t}+\beta * \tilde{\zeta} / c)\right]
$$

and

$$
\tilde{\zeta}=\frac{-\beta c \delta_{w}^{2} \gamma^{*}}{8 \Omega_{w}} \sin \left[2 \Omega_{w} \gamma^{*}(\tilde{E}+\beta * \tilde{\zeta} / c)\right] .
$$

For $\mathrm{k} \ll 1$ the ratio $\tilde{\mathrm{a}}_{\boldsymbol{\zeta}} / \mathrm{a}_{\mathrm{x}}$ of the amplitudes of the $\tilde{\boldsymbol{\zeta}}$ and $\tilde{x}$ oscillations is $\mathrm{K} / 8$ and the $\tilde{\zeta}$ motion is negligible. The $\tilde{\mathbf{x}}$ coordinate, then, executes simple harmonic oscillation with frequency $\widetilde{\omega}=\gamma^{*} \Omega_{w}$, and in the frame $\widetilde{F}$ the radiation is that of a simple oscillating dipole, linearly polarized and monochromatie with frequency $\widetilde{w}$. The dipole frequency observed in the laboratory frame, $F$, is related to $\tilde{\omega}$ by a Doppler shift,

$$
\omega_{1}=\frac{\tilde{\omega}}{\gamma *\left(1-\beta * \cos \theta_{0}\right)}
$$

where $\theta_{0}\left(\right.$ and $\phi_{0}$ ) are polar angles, in the laboratory frame, defining the radiation propagation vector with respect to the undulator axis. Since 


$$
\tan \theta_{0}=\frac{1}{\gamma^{\hbar}} \frac{\sin \tilde{\theta}}{\beta^{*}+\cos \tilde{\theta}}
$$

$\tilde{\theta}$ being the angle in the moving frame, the radiation is confined to small laboratory angles $\theta_{0} \sim 1 / \gamma$ and Eq. (265) becomes

$$
\omega_{1}=\frac{\Omega_{w}}{1-\beta^{*} \cos \theta_{0}} \simeq \frac{2 \Omega_{w} \gamma^{2}}{1+\frac{k^{2}}{2}+\gamma^{2} \theta_{0}^{2}}
$$

One notes that the radiation is not monochromatic in the laboratory frame and the frequency depends on the observation angle $\theta_{0}$.

On the other hand, when $K$ is not very small compared to unity, the $\tilde{\zeta}$ motion carnot be ignored and the electron trajectory in the moving frame $\widetilde{F}$ has the "figure-eight" shape sketched in Fig. 31. For large $\mathrm{K}$, the motion of the electron in the moving frame itself becomes relativistic, and the radiation, no longer that from a simple harmonic dipole, is synchrotron radiation with its many harmonic frequencies. That is, in $\widetilde{\mathrm{F}}$, and also in F, the spectrum comprises higher harmonics in addition to the fundamental frequency. The power in these higher modes is easily calculated. Averaged over one period, the power in the fundamental is

$$
P_{1}=\frac{4 \pi^{2}}{3} \frac{e^{2} c \gamma^{2}}{\lambda_{0}^{2}} \frac{k^{2}}{\left(1+k^{2} / 2\right)^{2}}
$$

while the total power radiated is

$$
P_{t o t}=\frac{4 \pi^{2}}{3} \frac{e^{2} c \gamma^{2}}{\lambda_{0}^{2}} R^{2}
$$

The difference $P_{\text {tot }}-P_{1}$ is, of course, the power contained in the higher 


$$
\phi
$$


modes.

A calculation of the spectrum, Including higher harmonics, has been made by Alferov, Bashmakov, and Bessanov (1973), who showed that the energy radiated per electron per unit solid angle per unit frequency interval is given by [see discussion leading to Eq. (48)]

$$
\frac{d I(\omega)}{d \Omega}=\frac{e^{2} \omega^{2}}{4 \pi^{2} c}\left|\int_{-\infty}^{\infty} d t \underline{n} \times(\underline{\underline{n}} \times \underline{B}(t)) e^{I \omega\left(t-\frac{\underline{\underline{n}} \cdot \underline{\underline{r}(t)}}{c}\right)}\right|^{2},
$$

in which Gaussian units are employed. In this equation, $\underline{I}(t)$ Is the electron trajectory of Eq. (262), $\underline{B}(t) \equiv \underline{\dot{\underline{x}}}(t) / c$, and $\underline{\mathrm{n}}$ is the radiation direction with respect to the undulator axis. The latter may be specified by the polar coordinates $\theta_{0}, \phi_{0}$, with $\phi_{0}=0$ corresponding to the plane of the electron motion and $\theta_{0}=0$ to the forward direction along the undulator axis. Thus,

$$
\underline{\underline{n}}=\left(\sin \theta_{0} \cos \phi_{0}, \sin \theta_{0} \sin \phi_{0}, \cos \theta_{0}\right)
$$

The integrals in (269) can be expressed in terms of Bessel functions. We make use of the identfty

$$
e^{-i 2 \sin \psi}=\sum_{n=-\infty}^{\infty} e^{-\ln \psi} J_{n}(z)
$$

for some variable $z$. If we define

$$
\xi_{z} \equiv \frac{\mathrm{k}^{2}}{4\left(1+\frac{\mathrm{K}^{2}}{2}+\gamma^{2} \theta_{0}^{2}\right)}, \xi_{x} \equiv \frac{2 \mathrm{R} \gamma \theta_{0} \cos \phi_{0}}{1+\frac{\mathrm{R}^{2}}{2}+\gamma^{2} \theta_{0}^{2}}
$$

and in turn

$$
s_{1} \equiv \sum_{n=-\infty}^{\infty} J_{n}\left(k \xi_{z}\right) J_{2 n+k}\left(k \xi_{x}\right)
$$




$$
s_{2} \equiv \sum_{n=-\infty}^{\infty} n J_{n}\left(k \xi_{z}\right) J_{2 n+k}\left(k \xi_{x}\right) \text {, }
$$

then for an undulator comprised of $\chi$ perlods,

$$
\frac{d I(\omega)}{d \Omega}=\frac{e^{2} \gamma^{2} h^{2}}{c} \sum_{k=1}^{\infty} G_{k}\left(R, \gamma \theta_{0}, \phi_{0}\right) H_{k}\left(\omega / \omega_{1}\right),
$$

where $k$ is the harmonic number of frequencies in the spectrum. In Eq. (271), the analog of Eq. (51) for ordinary synchrotron radiation,

$$
\begin{array}{r}
G_{k}\left(k, \gamma \theta_{0}, \phi_{0}\right)=\frac{4 k^{2}}{\left(1+\frac{k}{2} k^{2}+\gamma^{2} \theta_{0}^{2}\right)^{2}}\left\{\left[s_{1} \gamma \theta_{0} \cos \phi_{0}-\left(s_{1}+\frac{2}{k} s_{2}\right) \frac{1+i_{1} k^{2}+\gamma^{2} \theta_{0}^{2}}{2 \gamma \theta_{0} \cos \phi_{0}}\right]^{2}\right. \\
\left.+\left(\gamma \theta_{0}\right)^{2} s_{1}^{2} \sin ^{2} \phi_{0}\right\}
\end{array}
$$

and

$$
H_{k}\left(\frac{\omega}{\omega_{1}}\right)=\frac{\sin ^{2}\left[n \pi\left[\frac{\omega}{\omega_{1}}-k\right)\right]}{\pi^{2} x^{2}\left(\frac{\omega}{\omega_{1}}-k\right)^{2}} \text {. }
$$

Note that through $\omega_{1}$, [defined in Eq. (267)], $H_{k}$ depends on $K$ and on the product $r \theta_{0}$ :

The spectrum specified by Eqs. (271 - 273) was derived using an approximation valid for $\chi$ large. If we now consider $\chi^{+\infty}$,

$$
w_{k}+\frac{\omega_{1}}{n} \delta\left(\omega-k w_{1}\right) .
$$

For $\theta_{0} \rightarrow 0$, the dominant term of (272) is that with the factor 
$\left(s_{1}+\frac{2}{k} s_{2}\right)^{2}$, and one finds that the central energy intensity for a bandwidth of unit photon frequency is given by

$$
\left.\frac{d I(\omega)}{d \Omega}\right|_{\substack{\theta_{0}=0 \\ w=k w_{1}}}=\frac{n^{2} e^{2} r^{2}}{c} F_{k}(K)
$$

with

and

$$
F_{k}(K) \equiv \frac{k^{2} k^{2}}{\left(1+\frac{k^{2}}{2}\right)^{2}}\left[\frac{J_{k+1}}{2}\left(\frac{k k^{2}}{4+2 k^{2}}\right)-\frac{J_{k-1}}{2}\left[\frac{k k^{2}}{4+2 k^{2}}\right)\right]_{\text {for } k \text { odd }}^{2}
$$

$$
F_{k}(k) \equiv 0 \quad \text { for } k \text { even. }
$$

The frequency spectrum contains all the harmonics $k w_{1}$, where $w_{1}$ Is the frequency of the dipole mode given In Eq. (267). In the forward direction the spectrum is coinposed of odd harmonics only,

$$
\lambda_{k}=\frac{\lambda_{0}}{2 k \gamma^{2}}\left(1+\frac{k^{2}}{2}\right), \quad(k=1,3,5, \ldots)
$$

where the wavelengths $\lambda_{k}$ are related to the frequencies $\omega_{k}$ by $\lambda_{k}=$ $2 \pi c / \omega_{k}$. In the forward direction the width of the spectral peaks is determined by the $\mathrm{H}_{k}$ factor and is

$$
\left.\frac{\Delta \lambda}{\lambda_{k}}\right|_{\theta_{0}=0} \approx \frac{1}{\eta_{k}}
$$

If one accepts radiation within a cone of finite spread about the forward direction, the spectral peaks are broadened. For a radiation cone of halfangle $\bar{\theta}_{0}$, the spread in wavelengths becomes 


$$
\frac{\Delta \lambda}{\lambda_{k}} \approx \frac{\gamma^{2} \cdot \dot{\theta}_{0}^{2}}{1+\frac{k^{2}}{2}}
$$

More accurately, the width of a peak is the convolution of (279) with the "natural 1Inewidth" (278). The linewidth will not be increased too much bejond its minimal value (278) as long as

$$
\bar{\theta}_{0} \leqslant \frac{1}{r \sqrt{n}}
$$

This requirement is of course not met unless the angular divergence of the electron beam in the undulator is equally small, which means that the amplitude functions, $\beta_{z}$ and especially $\beta_{x}$ [Eq. (87)], cannot be too small at the undulator location. Values for the $B$ function in the insertion region shown in Fig. 14 are undesirably small for this purpose; they can be increased, without adversely affecting other regions of the ring, by change of focusing quadrupole fields and machine tune values.

From Eq. (275) one obtains for the central intensity of the k-th odd harmonic at wavelength $\lambda_{k}$

$$
4.56 \times 10^{6} \eta^{2} \gamma^{2} d F_{k}(K)
$$

photons $/ \mathrm{sec}, 1 \%$ bandwidth, $(0.1 \mathrm{mrad})^{2}$, current $\mathcal{Q}$ amperes, where $F_{k}(K)$ is defined in Eq. (276) and is plotted in Fig. 32. This result should be compared with that for a bending magnet source [Eq. (51)], which, in the same units, is

$$
3.46 \times 10^{5} \gamma^{2} \vartheta\left(\frac{\omega}{\omega_{c}}\right)^{2} \mathrm{k}_{2 / 3}^{2}\left(\frac{\omega}{2 \omega_{c}}\right)
$$

with $\omega_{c}=\frac{3}{2}(c / p) r^{3}$, the critical frequeacy. An undulator with $\chi=50$ 
periods has a central intensity exceeding that of the bending magnet source by four orders of magnitude.

To obtain a quantitative understanding of the undulator spectrum, we consider the example

$$
\lambda_{0}=5 \mathrm{~cm}, r=1370(\mathrm{E}=700 \mathrm{Mev}), \quad \chi=50,
$$

and assume the magnetic fleld can be varied between 0 and $4 k G$, so that from Eq. (259)

$$
0<K<2 \text {. }
$$

From Fig. 32 we see that a wavelength range of $400^{\circ}$ to $400 \AA$ is covered by varying the magnetic field and using the first, third, and fifth harmonics. of course, one eliminates the unwanted harmonics in any experiment by using appropriate optical elements. Eq. (281) shows that one can obtain on the order of $10^{16}$ photons/sec, amp, with $1 \%$ bandwidth at $\lambda_{k}$ in a forward cone of half-angle $0.1 \mathrm{mrad}$. To have this intensity from a bending magnet at $12 \mathrm{kG}$ one must accept 50 mrad of arc.

As prevlously noted, Eq. (279), the spectral peaks are broadened when one accepts radiation over a cone of finite half-angle $\bar{\theta}_{0}$. This effect is shown in Fig. 33, a plot of a function based on Eqs. (271-273),

$$
I_{\bar{\theta}_{0}}(\omega)=\int_{0}^{\bar{\theta}_{0}} d \theta_{0} \sin \theta_{0} \int_{0}^{2 \pi} d \phi_{0} \frac{d I(\omega)}{d \Omega} ;
$$

for the plot the function has been evaluated for $k=1$, for $\gamma \bar{\theta}_{0}=0.1$ and 0.4 , and for $w$ in the region of the first harmonic. It is clear that if the undulator $K$ is tuned so that at $\bar{\theta}_{0} \sim 0^{0}$ the peak of the spectrum is located at the wavelength of interest, there is little to be gained other than extraneous radiation of longer wavelengths by increase of the 


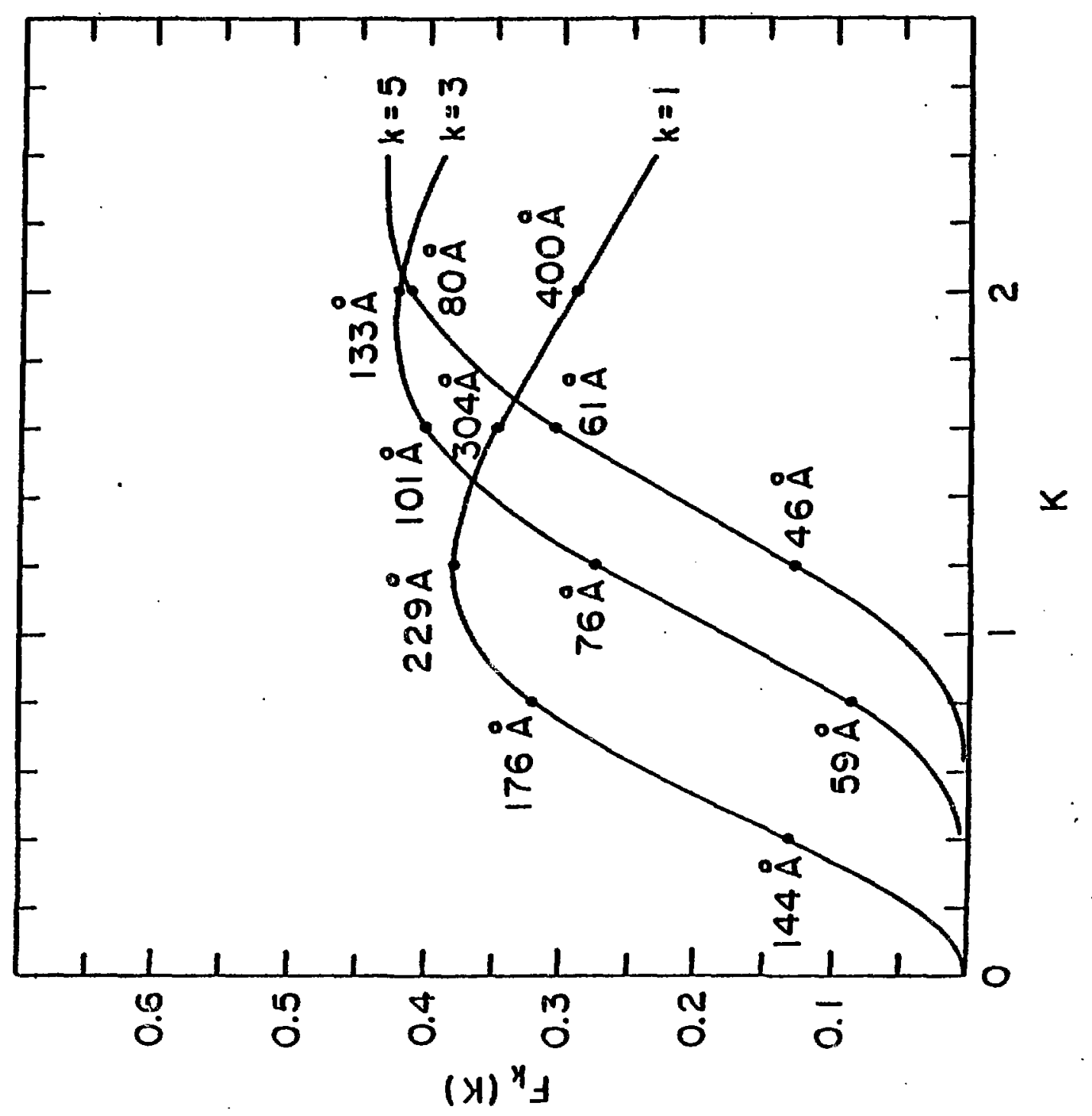




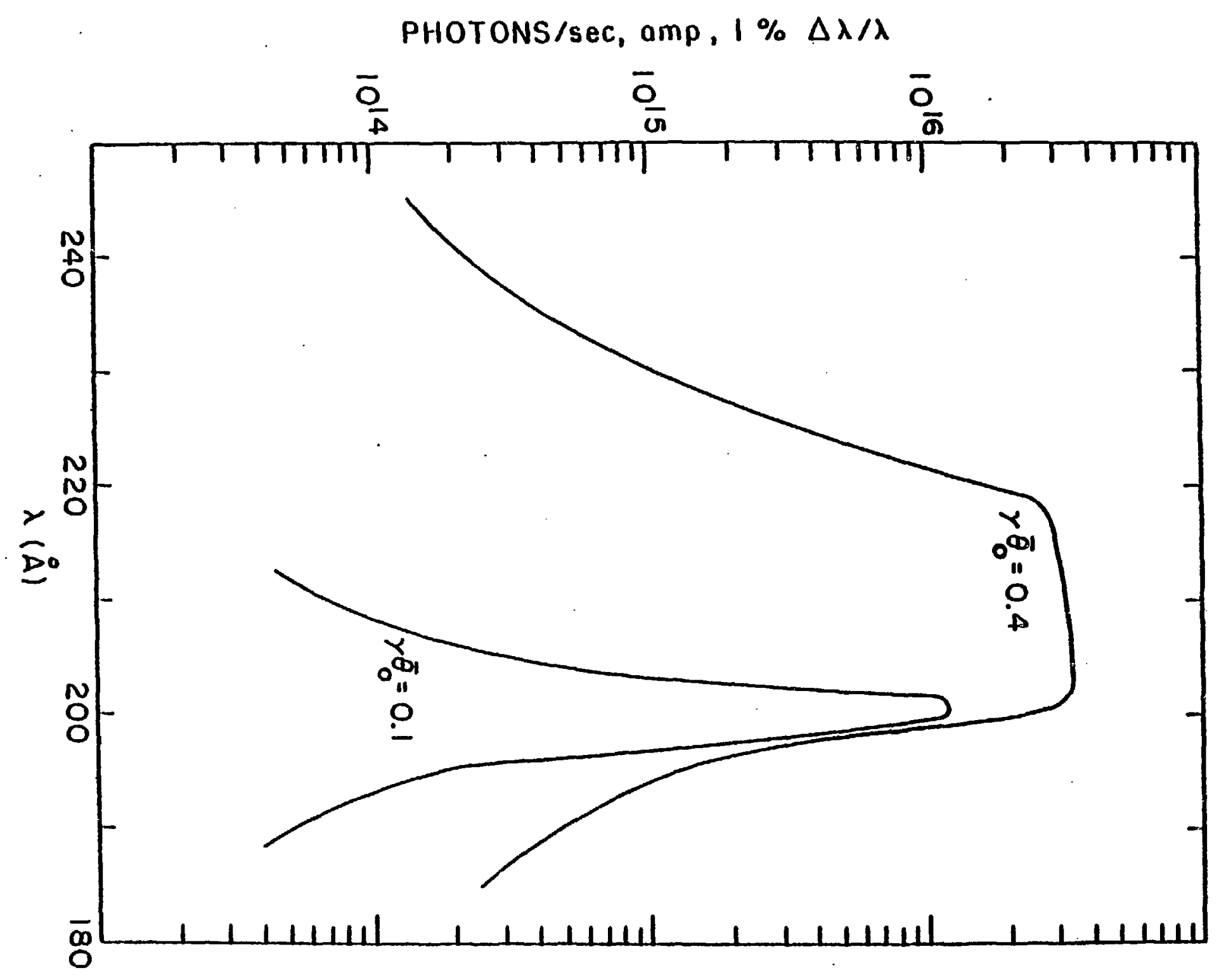


acceptance angle beyond $\bar{\theta}_{0} \sim 0.1 / \gamma$ radians. There are a few experiments for which the broader spectral width associated with larger $\bar{\theta}_{0}$ is useful. Plots of the spectrum integrated over angle,

$$
I(\omega)=\int d \Omega \frac{d I(\omega)}{d \Omega},
$$

are displayed in Fig. 34 (as a function of wavelength) for three $R$ values: $0.4,1$ and 2. In the calculations for these plots, the limiting form for $\mathrm{H}_{k}$, Eq. (274), was used. Even in these spectra, peaks of substantial amplitude remain. In Fig. $35 \mathrm{w}$ sketch, for $\mathrm{K}=1$, the angular distributions of radiation for several harmonics,

$$
I\left(\theta_{0} \phi_{0}\right)=\int d \omega \frac{d I(\omega)}{d \Omega} \text {, }
$$

at $\phi_{0}=0^{\circ}$, i.e. In the plane of the electron orbit, and $\phi_{0}=90^{\circ}$, 1.e. perpendicular to it. Here again the approximation (274) was used. Two aspects of the angular behavior are noteworthy: even harmonics vanish In the forward direction but contribute at other angles; and in the undulation plane the higher harmonics, both odd and even, exhibit oscillations in intensity with change of observation angle.

The total energy radiated by an electron passing through an undulator having a period length $\lambda_{0} \mathrm{~cm}$ is

$$
\mathrm{U}(\mathrm{keV})=1.9 \times 10^{-9} n \mathrm{r}^{2} \mathrm{k}^{2} / \lambda_{0}
$$

and the radiated power for amperes is

$$
P_{\text {tot }} \text { (watts) } \approx 1.9 \times 10^{-6} \eta \gamma^{2} K^{2} s / \lambda_{0} .
$$

For $\mathrm{k}=1, \eta=50, \lambda_{0}=5 \mathrm{~cm}, \gamma=1370(700 \mathrm{MeV})$, and $\psi=1$ amp the power 


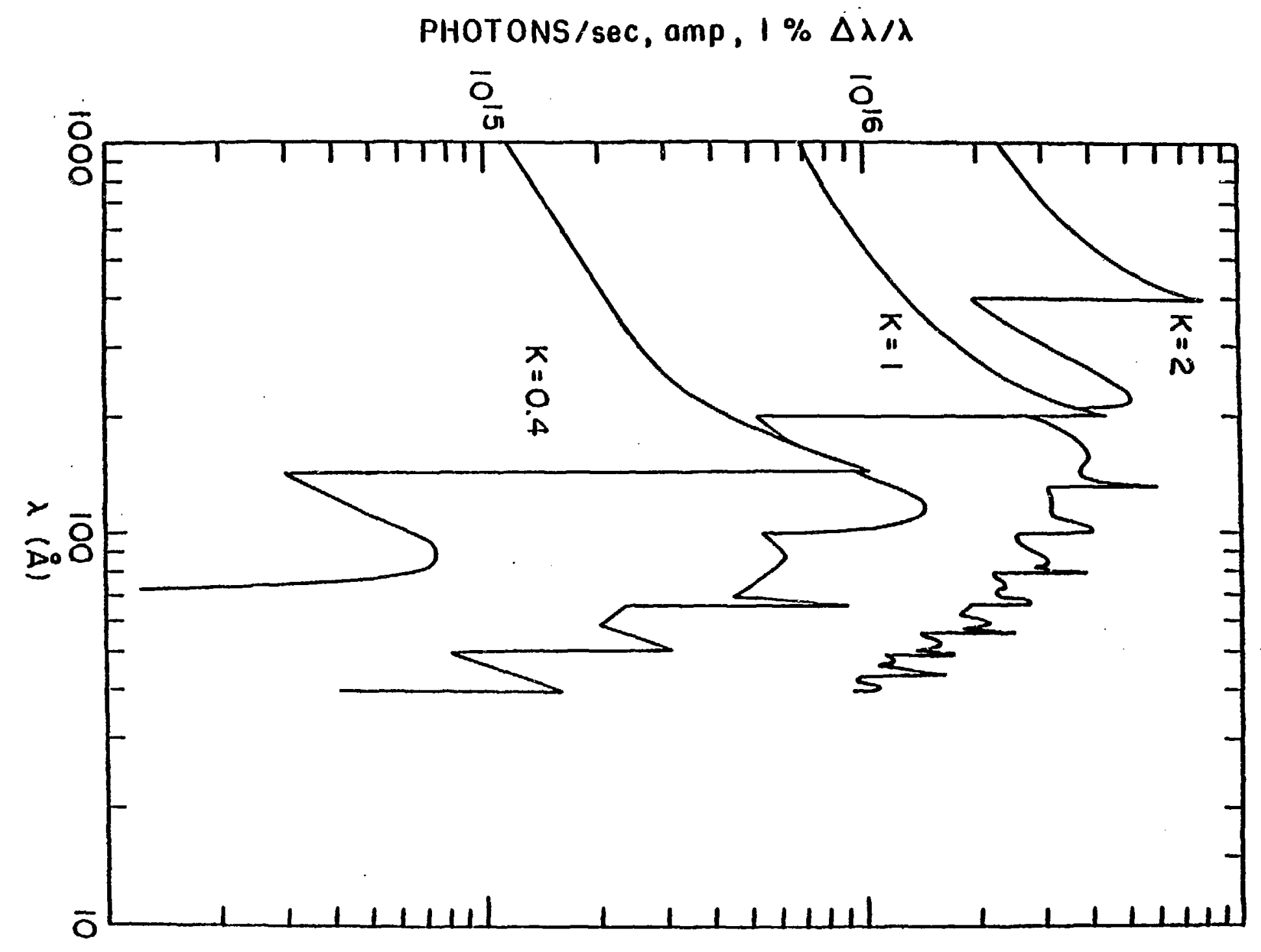




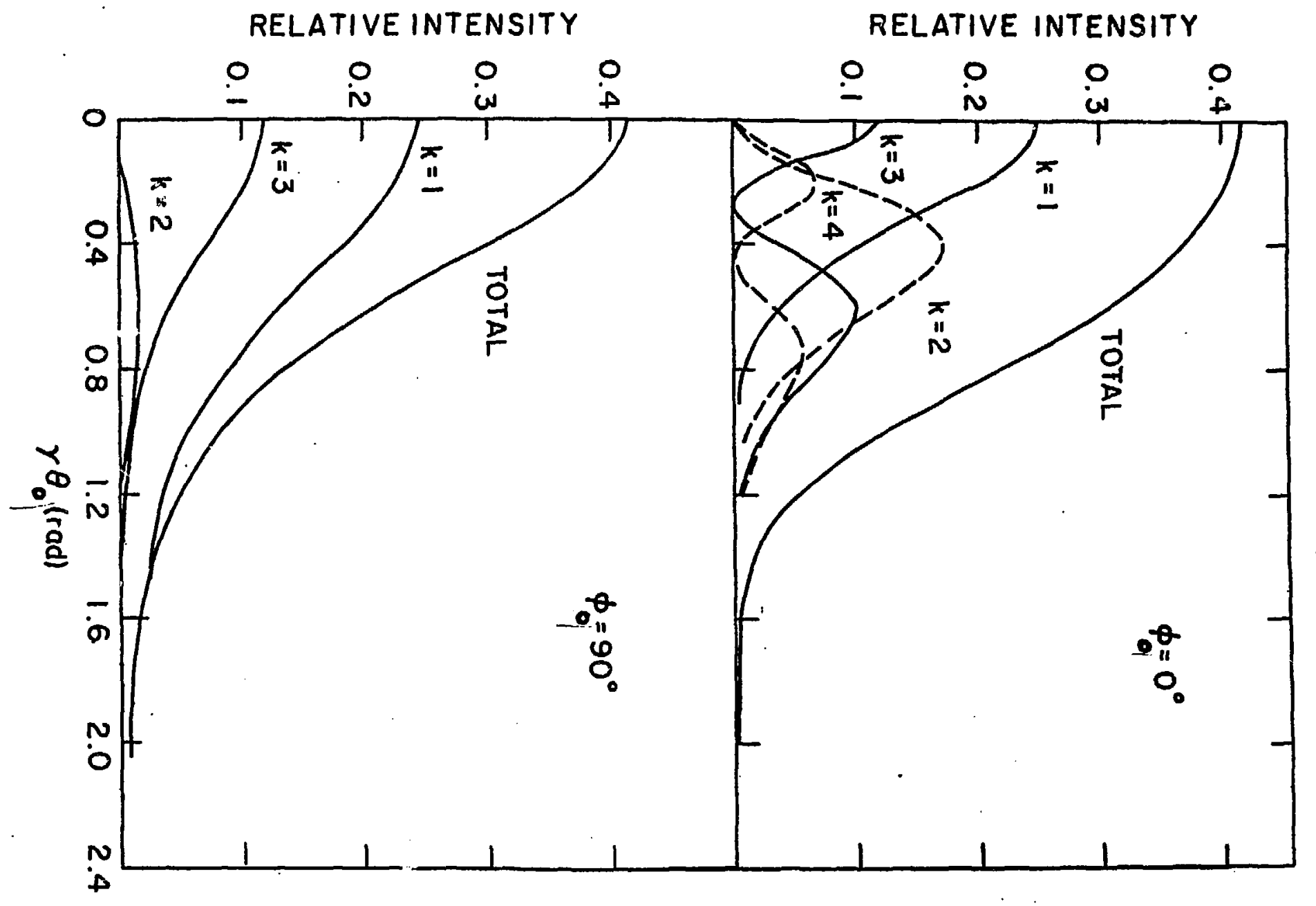


would be 36 watts. In Fig. 36 there are shown plots of the fraction of energy radiated into a cone of half-angle $\bar{\theta}_{0}, 1 . e .$,

$$
\frac{1}{\bar{U}} \int_{0}^{\bar{\theta}_{0}} d \theta_{0} \sin \theta_{0} \int_{0}^{2 \pi} d \phi_{0} \int d \omega \frac{d I(\omega)}{d \Omega},
$$

as a function of $r \bar{\theta}_{0}$, for $K=0.4,1$ and 2 .

A few of the salient characteristics of transverse undulators deserve to be summarized. For a given magnet structure and a flxed electron energy, the frequency spectrum can be tuned by varying the magnetic field strength $B_{0}$. As shown in Figs. 32 and 34, Increasing $B_{0}$ (hence Increasing $\mathrm{K}$ ) shifts an Individual peak in the frequency spectrum to a longer wavelength. Although a given peak is thus softened, the heights of peaks corresponding to higher harmonics increase with increasing $B_{0}$ and, in the spectrum as a whole, more hard photons are emitted.

The power radiated, and thus the total photon flux, Increase linearly with the number $h$ of wiggle periods. However, it is clear from FIg. 34 that the frequency spectrum is not simply obtalned by scaling the result for a one-period device. When $T$ Is large, the spatial perlodicity of an undulator field alters the photon flux distribution so as to enhance $1 t$ at certain wavelengths and depress it at others, relative to what one would expect from scaling the $\eta=1$ results.

Undulators being considered at the varlous synchrotron light facilities would generate photon spectra peaked at wavelengths longer than the wavelength, $\lambda_{c}$, characteristic of bending magnet spectra. For a ring operating at $3 / 4 \mathrm{GeV}$, an undulator with $\lambda_{0}=5 \mathrm{~cm}$ will produce radiation in the wavelength region from 40 to $400 \AA$; for a $2.5-\mathrm{GeV}$ machine the range, which scales as $1 / \gamma^{2}$, is 4 to $40 \AA$ for the same device. 


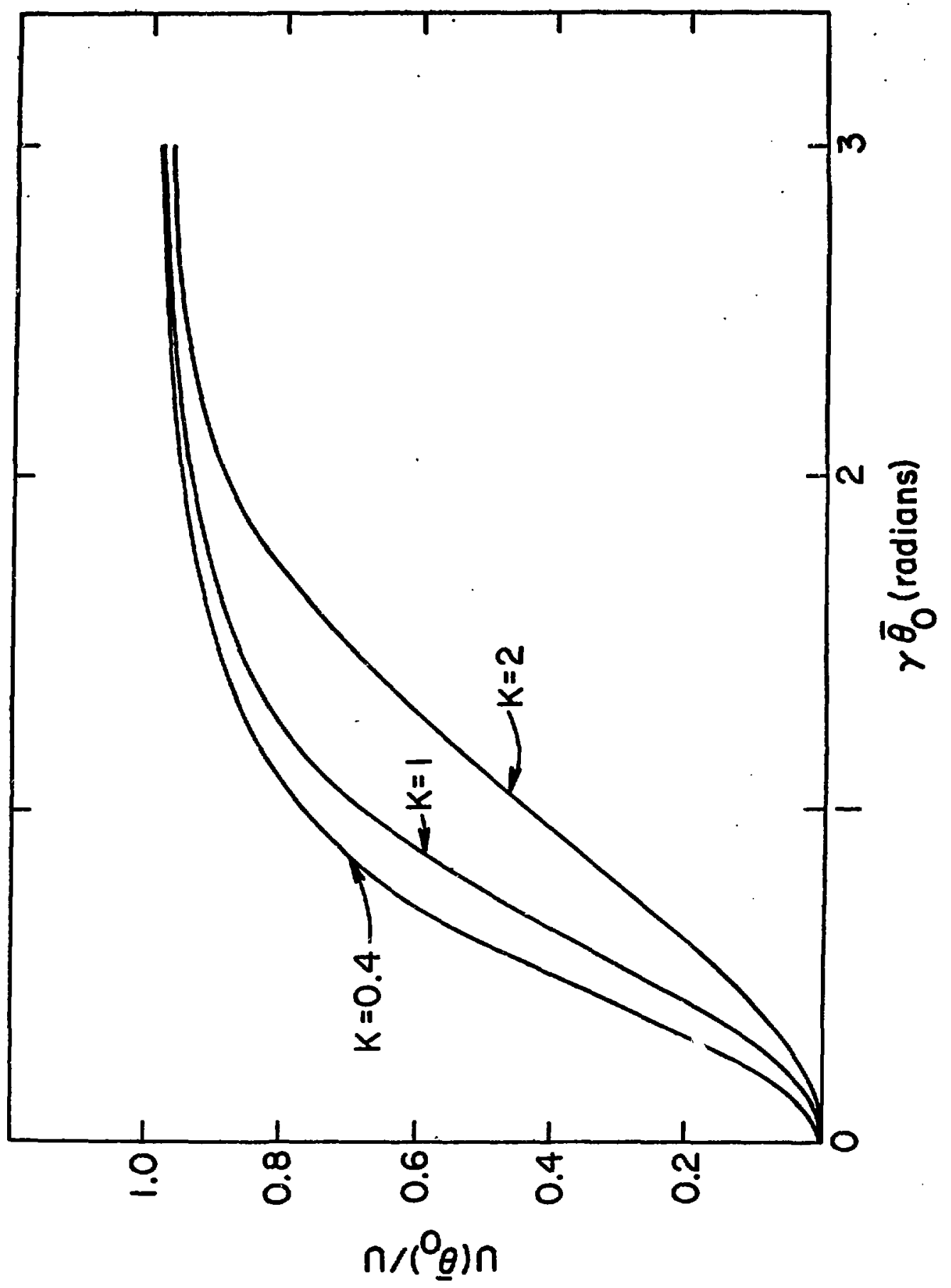


Achieving long wavelengths by decreasing $\gamma$ is useful within the 1Imits imposed by shortening of the Touschek 11fetime and by the tolerance af other users around the ring, whose photon beams will also be affected by lowering the electron energy. Longer wavelengths can be obtained also by increasing the undulator period $\lambda_{0}$, but spatial limitations may then require a decrease in the number of periods.

The total power radiated by an undulator is relatively small. One sees from Eq. (287b) that only 36 watts are radiated by a 50 period device operating at $\mathrm{K}=1$ with 1 Ampere of 3/4-GeV electrons. This may be compared with a power of $=2$ watts per milliradian of azimuthal orbit from a $12 \mathrm{kG}$ bending magnet on the same storage ring. For wavelengths near the harmonics $\lambda_{k}(k=1,3,5, \ldots)$, the central brightness of the undulator is extremely high, about $10^{4}$ times that of a bending magnet. This very large difference is consistent with the modest increase in power, because nearly all radiation over the narrow wavelength bands, $\lambda_{k}$ to $\lambda_{k}+\Delta \lambda$ (for $\mathbf{k}$ odd), is concentrated in a small cone about the forward direction.

If the accepted cone of radiation is restricted to an angle of about one-tenth of $\sigma_{R}$ at a bending magnet, one obtains a sharply peaked spectrum a few percent in width, as shown in Fig. 33. This peak narrows with $1 / x$, and 1ts height grows as $\chi^{\hat{2}}$. Thus, source brightness varies as peak helght, $n^{2}$, but power radiated into the narrow cone, which depends upon the peak area, Increases linearly with $\mathfrak{K}$.

It should be emphasized that the small forward cone angle required to obtain a very narrow spectral peak is defined with respect to electron direction. This angle therefore cannot be decreased beyond a .

limit set by the angular spread of the electron beam, $\sigma_{x}$, and $\sigma_{z}$, in 
the region of the undulator magnet. In fact, for obtaining a narrow peak the $x-x^{\prime}$ source ellipes (FIg. 19) for the insertion region at a tune ylelding small $\beta_{x}$ and $\beta_{z}$ there are not suitable. Smaller $\sigma_{x}$, with consequentiy larger $\sigma_{x}$, is required, and it may be achieved by adjusting the gradients in the quadrupoles byding the insertions. 


\subsection{Emission from an Undviator-Helical Maggetic Field}

Consider an open helix wound on the outside of a bore tube, and then a second helix wound in the spaces between the turns of the first. When the helices are connected so that a current takes opposite directions passing through them, the central longitudinal component of the magnetic field is cancelled, and a transverse periodic helical field of constant magnitude is produced on axis. A good approximation to the central field Is

$$
\underline{B}=B_{0}\left(e_{x} \cos \frac{2 \pi \zeta}{\lambda_{0}}+e_{z} \sin \frac{2 \pi \zeta}{\lambda_{0}}\right) \text {, }
$$

where the $e$ are unit vectors in the $x$ and $z$ directions and $\zeta$ is position along the axis. The electrons follow a helical orbit

$$
\underline{r}(t)=\underline{e}_{\zeta} \beta_{h}^{*} c t+\underline{e}_{x} a \sin \Omega_{h} t-\underline{e}_{z} a \cos \Omega_{h} t
$$

wlth traversal frequency

$$
\Omega_{h}=2 \pi \beta_{h}^{* c} / \lambda_{0} \sim \frac{2 \pi c}{\lambda_{0}}
$$

and radius of $:-z$ motion

$$
a=\mathrm{cK}_{\mathrm{h}} / \Omega_{\mathrm{h}} \gamma \text {. }
$$

In the above

$$
\mathrm{K}_{h} \equiv \frac{e B_{0} \lambda_{0}}{2 \pi m c^{2}} \cong \frac{B_{0}\left(\text { kilogauss) } \lambda_{0}(\mathrm{~cm})\right.}{10}
$$

$$
\text { and } \beta_{h}^{*} \equiv \beta \sqrt{1-K_{h}^{2} / \gamma^{2}} \text {. }
$$


Note that $\Omega_{h}, K_{h}$, and $\beta_{h}^{*}$ are eimilar to, but not identical with, their counterparts for the transverse undulator [Eqs. (256), (259), and (261)]. Unlike the case for transverse undulators, the longltudinal velocity 18 constant in tire or position in the undulator and is equal to Bt. The transverse velocity is

$$
\underline{B}_{\perp}=\frac{\mathrm{k}_{\mathrm{h}}}{\gamma}\left(\frac{\mathrm{e}}{\mathrm{x}} \cos \frac{2 \pi \zeta}{\lambda_{0}}+\underline{\mathrm{e}}_{\mathrm{z}} \sin \frac{2 \pi \zeta}{\lambda_{0}}\right) \text {. }
$$

An intuitive understanding of the spectrum is obtained by again following Motz (1951) and Purcell (1972) in considering a Lorentz transformation from the lab frame $F$ to a coordinate system $\widetilde{F}$ moving with velocity $\beta_{\mathrm{h}}^{*}$. There is, as already noted, no residual longitudinal motion in $\widetilde{\mathrm{F}}$, and in the $\widetilde{\mathrm{F}}$ frame the electron moves on a circular orbit with angular frequency $Y_{h}^{*} \Omega^{*}$ The relativistic parameter $Y_{h}^{*}=\left(1-\beta_{h}^{*}\right)^{-\frac{2}{2}}$ is related to the magnet parameters by

$$
Y_{h}^{*}=r\left(1+k_{h}^{2}\right)^{-\frac{d}{2}}
$$

which differs from Eq. (264).

In the weak field 1imit, $\mathbb{K}_{h} \ll 1$, the motion in $\widetilde{F}_{\text {is }}$ nonrelativisitic, and the radiation observed is circularly polarized and monochromatic with frequency $\tilde{\omega}=\gamma_{h}^{\star \Omega}$. Eq. (265) then yields the Doppler shifted frequency observed in the lab,

$$
\omega=\hat{w}_{1 h}=\frac{\Omega_{h}}{1-\beta_{h}^{\star} \cos \theta_{0}} \cong \frac{2 \Omega_{h} \gamma^{2}}{1+k_{h}^{2}+\gamma^{2} \theta_{0}^{2}}
$$

When the magnetic field strength $B_{0}$ is increased, higher modes contribute 
and the radiation observed in $\widetilde{F}$ is no longer monochromatic. The spectrum of radiation emitted has been calculated by Kincaid (1977) and AIferov, Bashmakcv, and Bessonov (1973), using Eq. (269), together with Eq. (294) . for the helical orbit. They find that the energy radiated per unit solid angle and unit frequency interval, in the laboratory frame, is

$$
\begin{aligned}
\frac{d I(\omega)}{d \Omega}= & \left.\frac{e^{2} \omega^{2} k_{h}^{2}}{\pi^{2} c \Omega_{h}^{2} \gamma^{2}} \sum_{k=1}^{\infty}\left[J_{k}^{\prime 2}(\xi)+\int \frac{\gamma_{0}}{k_{h}}-\frac{k}{\xi}\right)^{2} s_{k}^{2}(\xi)\right] \\
& \times \frac{\sin ^{2}\left[2 \pi\left(\frac{\omega}{\omega_{1 h}}-k\right)^{2}\right]}{\left(\frac{\omega}{\omega_{1 h}}-k\right)^{2}}
\end{aligned}
$$

where

$$
\xi=\frac{k_{h} \omega \theta_{0}}{r \Omega_{h}} .
$$

The spectrum consists of harmonics $k{ }_{1 h}$ of the dipolar frequency $\omega_{1 h}$ specified in Eq. (296).

Eqs. (297) and (298) show that for $\theta_{0}=0$ (forward direction) all the higher harmonics vanish, and one is left with a spectrum consisting of a single narrow peak at $\omega=\omega_{1 h}$. The central brightness is

$$
\left.\frac{d I(\omega)}{d \Omega}\right|_{\substack{\omega=\omega_{1 h} \\ \theta_{0}=0}}=\frac{2 \eta \eta^{2} e^{2} \gamma^{2} k_{h}^{2}}{c\left(1+k_{h}^{2}\right)^{2}}
$$

and, as stressed by Kincaid (1977), this expression is at a maximum when $\mathrm{K}_{\mathrm{h}}=1$, yielding 


$$
\left.\frac{d I(\omega)}{d \Omega}\right|_{\substack{\omega=\omega_{1 h} \\ \theta_{0}=0 \\ k_{h}=1}}=\frac{\varkappa^{2} \mathrm{e}^{2} \gamma^{2}}{2 \mathrm{c}} .
$$

This contrasts with the result for the transverse undulator spectrum, which, at $\theta_{0}=0$, Includes all odd harmonics. A helical undulator, like the transverse device, can produce a beam of high brightness; at the frequency of the first harmonic, all the radiation in a narrow band $\Delta \omega / \omega \sim 1 / n$ is confined to a very small axial cone $\bar{\theta}_{0} \sim 1 /\left(\gamma h^{\frac{1}{2}}\right)$. For values of $k_{h}<1$ most of the power is in the first harmonic and is directed forward; with increasing $K_{h}$, higher harmonic content increases and this emission is into a conical shell at angle $\theta_{0} \sim K_{h} / \gamma$ and shell thickness $\Delta \theta_{0} \sim 1 / \gamma$. Infection of beam into a storage ring fitted with a helical wiggler is more difficult than is the case with a transverse wiggler of the same period length $\lambda_{0}$. In a helical wiggler, the bore, through which the orbit chamber passes, must be of diameter roughly one-half $\lambda_{0}$. For a transverse device, the vertical separation between magnet poles is similarly restricted but the width can be made large in order to better accommodate horizontal betation oscillations at infection.

At $\theta_{0} \sim 0$ and $K \leq 1$ transverse and helical undulators are similar with respect to brightness and 1 ts variation with $n$. However, the TW beam is linearly polarized, and HW's give forth a beam having clrcular polarization. Helicalwigglers operated at $\mathrm{K}<1$ have the desirable property of producing, on axis, a bright, quasimonochromatic beam, but the wavelength can be varied over only a limited range by change of wiggler field. 
For a more detailed discussion of the spectrum of the helical undulator, we refer the reader to the paper of KIncald (1977). The reader may also wish to consult the article of Blewett and Chasman (1977), who studied the properties of electron trajectories in a helical magnetic fleld, and who considered some problems assoclated with inserting a hellcal wigler into a storage ring. 


\subsection{The Free Electron Laser}

The free electron laser (FEL) consists of an undulator within an optical cavity, as illustrated in Fig. 37. An electron beam, moving under the combined influence of the static undulator field and the radiation field, does net work on the cavity radiation field amplifying its Intensity. Optical gain was observed by Elias et al. (1976), who measured the amplification of $10.6-\mu m$ infrared radiation from a carbon-dioxide - laser by the 24-MeV bunched electron beam of the Stanford University superconducting linear accelerator (Fig. 38). About one year later Deacon et al. (1977) achieved the operation of an FEL oscillator. As we shall see later in this section, Eqs. (330 and 331), for fixed undulator parameters $\lambda_{0}, B_{0}$, and $h$, the optical gain is proportional to the product of the peak current of the electron beam and the radiation wavelength, $\lambda$, raised to the $3 / 2$ power. At $10.6 \mu \mathrm{m}$ a laser gain of $7 \%$ was attained with a peak current of about $70 \mathrm{ma}$; in order to achieve a comparable gain at $0.3 \mu \mathrm{m}$, a peak current on the order of 10 Amp would be necessary. Hence, an infrared free electron laser can be made to operate with the electron beam from a Innac or microtron, but for an FEL in the ultraviolet one requires a current accumulator, such as a storage ring. There is reason to hope that the FEL will prove to be a powerful source of continuousiy tunable, coherent electromagnetic radiation in the wavelength region extending from about $0.1 \mu \mathrm{m}$ to $10 \mu \mathrm{m}$.

We shall not discuss the original quantum mechanical description of the FEL developed by Madey (1971) but instead present briefly the work of Colson $(1977,1978)$, following the exposition of Pellegrint (1979). 


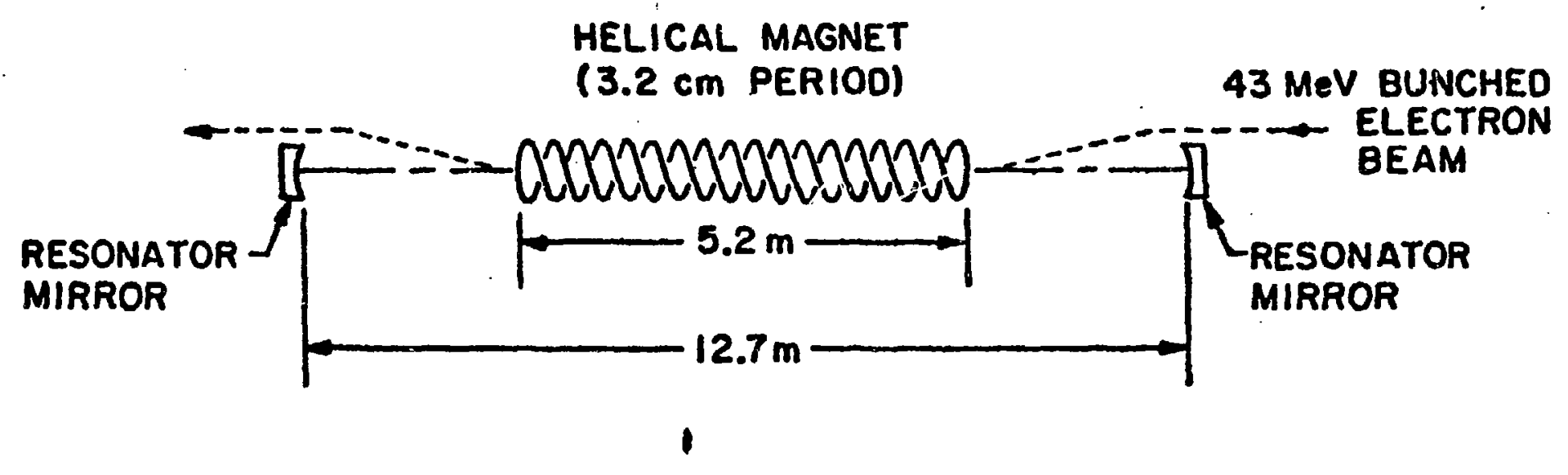

Fig 37 


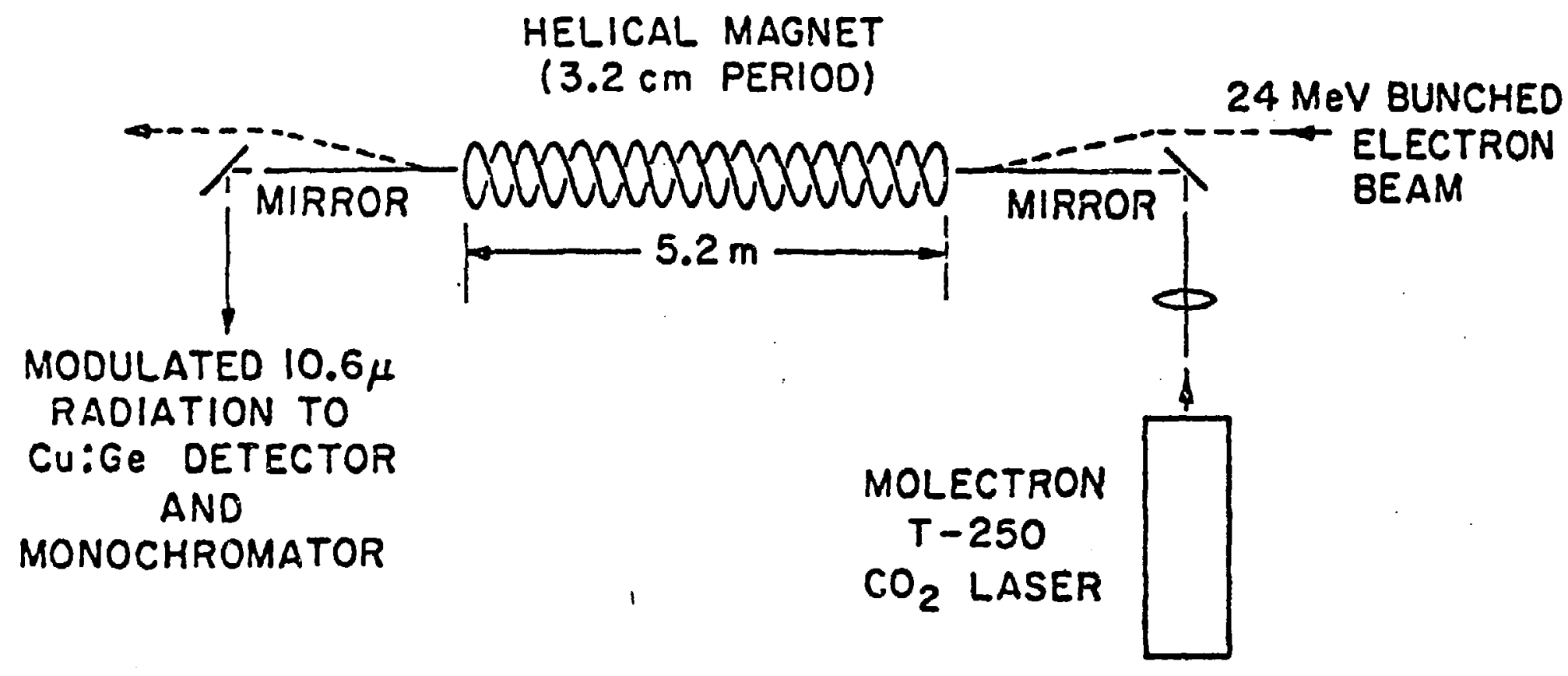


133

Consider an electron moving both in the field of a helical wiggler [Eq. (289)],

$$
\underline{B}=B_{0}\left\{e_{x} \cos \frac{2 \pi \zeta}{\lambda_{0}}+e_{z} \sin \frac{2 \pi \zeta}{\lambda_{0}}\right\}
$$

and in the radiation field

$$
\begin{aligned}
& \underline{E}_{r}=E_{0}\left\{e_{x} \sin \left(\frac{2 \pi \zeta}{\lambda}-\omega t+\Phi_{0}\right)+e_{z} \cos \left(\frac{2 \pi \zeta}{\lambda}-\omega t+\Phi_{0}\right)\right\}, \\
& \underline{B}_{r}=e_{\zeta} x \underline{E}_{r} .
\end{aligned}
$$

The Lorentz equation of motion is

$$
\frac{d}{d t}(\gamma \underline{B})=\frac{e}{m c}\left[\underline{E}_{r}+\underline{B} \times\left(\underline{B}+\underline{B}_{r}\right)\right]
$$

and as a consequence the rate of change of the electron energy is

$$
\dot{\gamma}=\frac{\mathrm{e}}{\mathrm{m} c} \underline{\mathrm{B}} \cdot \underline{\mathrm{E}} \mathrm{r}
$$

If the radiation field is not too strong, it is reasonable to approximate the electron trajectory by Eq. (290), the result in the static undulator field. In this case the magnetic field does essentially no work on the electron, but it does give the electron trajectory a small transverse velocity $\underline{B}_{\perp}$, so that the transverse radiation electric field, $\underline{E}_{r}$, can do work at a rate proportional to $\underline{B}_{\perp} \cdot E_{-r} \cdot$ From (290) we see that 
the electron velocity in the absence of the radiation field is

$$
\underline{B}=B_{h}^{*} \underline{e}_{\zeta}+\underline{B}_{\perp}
$$

where from Eq. (293), if one keeps terms of order $\frac{1}{\gamma^{2}}$,

$$
B_{h}^{*} \approx 1-\frac{1+K_{h}^{2}}{2 \gamma^{2}}
$$

and from Eq. (294),

$$
\underline{\beta}_{\perp}=\frac{k_{h}}{\gamma}\left\{e_{x} \cos \frac{2 \pi \zeta}{\lambda_{0}}+e_{z} \sin \frac{2 \pi \zeta}{\lambda_{0}}\right\}
$$

Inserting the expressions (302) for $\underline{E}_{\Gamma}$ and (308) for $\underline{\beta}_{\perp}$ into Eq. (305), we find

$$
\dot{\gamma}=-\frac{e E_{0} R_{h}}{m c \gamma} \sin \dot{\phi}_{L}
$$

with the phase $\Phi_{\mathrm{L}}$ defined as

$$
\Phi_{L}=\left(\frac{2 \pi}{\lambda}+\frac{2 \pi}{\lambda_{0}}\right) \zeta-\omega t+\Phi_{0}+\pi
$$

where $\Phi_{0}$ is the phase of the radiation field [Eq. (302)]. Since $\dot{\zeta}=\beta_{h}{ }^{*} c$, with (307) and $\omega=2 \pi c / \lambda$ we see that

$$
\dot{\phi}_{L} \approx \frac{2 \pi c}{\lambda_{0}}\left[1-\frac{\lambda_{0}}{\lambda} \frac{1+k_{h}^{2}}{2 \gamma^{2}}\right]
$$


It is apparent that there can be a net transfer of energy between the electron and the EM wave, provided that $\underline{B}$ and $\underline{E}_{-r}$ remain in phase over the length of the magnet. Eq. (309) shows that this is the case when $\dot{\phi}_{L}$ is zero or is suffictently small. For a given radiation wavelength, $\lambda=2 \pi c / \omega$, a resonant electron energy $\gamma_{r}$ can thus be defined by the condition $\dot{\phi}_{L}=0$. From Eq. (311) this is

$$
\gamma_{r}^{2}=\frac{\lambda_{0}}{2 \lambda}\left(1+R_{h}^{2}\right)
$$

The physical meaning of the resonance condition (312) can be understood by noting that an electron takes the time $\lambda_{0} / \beta_{h}{ }^{*} c$ to traverse one pertod of the undulator, while the $E M$ wave makes the transit in time $\lambda_{0} / c$. At resonance the difference between chese transit times equals one period $\tau=\lambda / c$ of the $E M$ wave, 1.e.

$$
\tau=\lambda / c=\lambda_{0}\left(\frac{1}{B_{h}{ }^{*} c}-\frac{1}{c}\right)=\frac{\lambda_{0}}{c}\left(\frac{1+K_{h}^{2}}{2 \gamma_{r}^{2}}\right)
$$

Under the condition that the energy transfer is small, the same phase relation which exists for one period of the undulator between the electron velocity and the EM wave is repeated in all subsequent periods. One should note that (312) can be written

$$
\lambda=\frac{\lambda_{0}}{2 \gamma_{r}^{2}}\left(1+K_{h}^{2}\right),
$$

which is the relation (296) for wavelength $\lambda$ radiated spontaneously on axis by an electron of energy $\gamma_{r}$ passing through the undulator. 
If again we assume that the electron energy change in traversing the undulator is small and that the initial electron energy is near the resonant value $\gamma_{I}$, then Eqs. (309) and (311) can be simplified. Defining

$$
\eta_{L}=\frac{\gamma-\gamma_{I}}{\gamma_{r}}
$$

and taking $\eta_{L} \ll 1$, we have

$$
\dot{\eta}_{L}=-\frac{e E_{0} K_{h}}{m c \gamma_{r}{ }^{2}} \sin \Phi_{L}
$$

and, making use of (312),

$$
\dot{\phi}_{L}=\frac{4 \pi c}{\lambda_{0}} \cdot n_{L}
$$

These equations have the same forms as those describing synchrotron oscillations in a storage ring when the energy loss per revolution to synchrotron radiation is negligible, $U_{\text {rad }}(\varepsilon)=0$ [see Eqs. $(109)-(111)$ ]. Combining Eqs. (315) and (316), we obtain

$$
\ddot{\Phi}_{L}=-\Omega_{L}^{2} \sin \phi_{L}
$$

where the square of the small araplitude oscillation frequency is

$$
\Omega_{L}^{2}=\frac{4 \pi e E_{0} K_{h}}{\lambda_{o} r_{r}{ }^{2}}
$$


The first-integral of the motion is given by

$$
\frac{1}{2} \dot{\phi}_{L}^{2}-\frac{1}{2} \dot{\phi}_{L O}^{2}=\Omega_{L}^{2}\left(\cos \Phi_{L}-\cos \phi_{L O}\right),
$$

in which $\Phi_{\text {Lo }}$ and $\dot{\Phi}_{\text {Lo }}$ represent the initial conditions.

Eq. (317) can be thought of as the equation of motion of a pendulum, and frow the first-integral (319) one obtains the well-known phase space trajectories illustrated In Fig. 39. The closed trajectories correspond to bounded oscillations; the open curves lying above and below the separatrices correspond to the pendulum swinging completely around. We shall see that it is these open trajectorles which are important in explaining the gain of the free electron laser.

Whether energy is transferred from the electron to the wave (emission) or from the wave to the electron (absorption) depends on the spatial position of the electron relative to the wave on the scale of the radiation wavelength. Since the electron bunch is long compared to this wavelength, the electrons are initlally uniformly distributed in phase. However, the Initial energy distribution or, equio =1 cat.1y, $\dot{\phi}_{L}$ distribution can be very narrow.

We consider a bunch of electrons uniformly distributed in phase and all at initial energy $\gamma_{0}$. The laser gain is determinsed by the change $\Delta \gamma$ in the electron energy averaged over the initial distribution

$$
\left\langle\frac{\Delta \gamma}{\gamma_{0}}\right\rangle=\frac{1}{2 \pi} \int_{0}^{2 \pi} \mathrm{d} \Phi_{L 0} \frac{\Delta \gamma\left(\Phi_{L_{0}} \dot{\phi}_{L 0}\right)}{\gamma_{0}}
$$




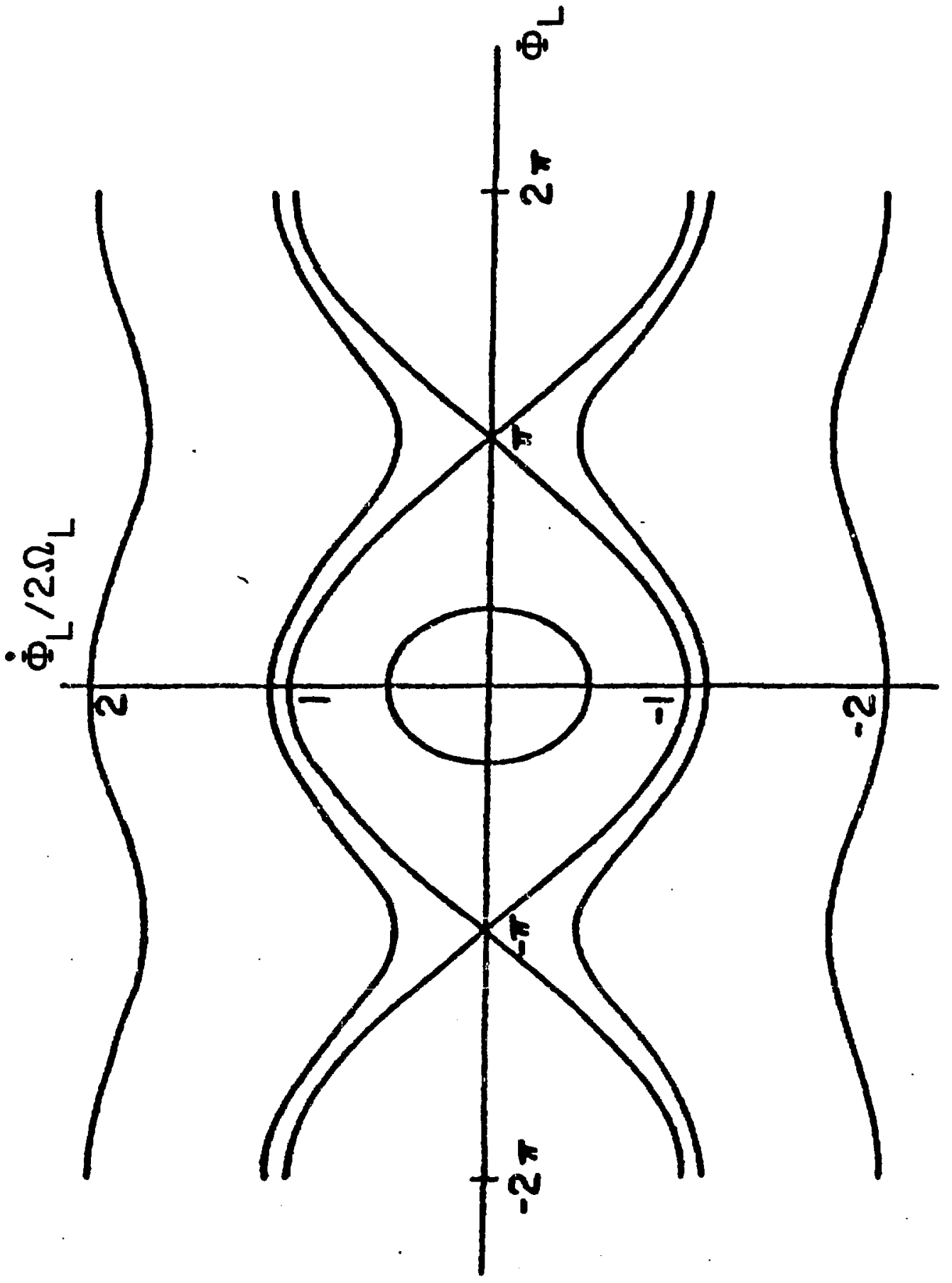


For $\dot{\phi}_{L O}=0$ (1.e. $\gamma_{0}=\gamma_{r}$ ) calculations show [Colson $(1977,1978)$ ] that for every electron which loses energy there is a corresponding electron vhich gains the same amount, with the result that the net energy transfer or gain vanishes. However, when the radiation fleld is not too strong, one can choose a value $\gamma_{0}>\gamma_{r}$ such that there is a net gain of energy by the $\mathrm{EM}$ wave from the electron beam. This results from dynamical effects which increase the number of electrons having phase proper to do work on the radlation field and decrease the number having phase proper to absorb energy. Basic to this behavior are two facts: first, electrons initially at energy $\gamma_{0}>\gamma_{r}$ which gain energy move away from the resonance energy $\gamma_{r}$, while those losing energy move closer; and second, the rate of phase change is more rapid further from resonance, as stated in Eq. (316). Therefore, while traversing the undulator, those electrons Initially in a thase region to absorb energy from the radiation, migrate relatively rapidly toward the phase region corresponding to emission while the inftially emitting electrons leave their phase reglon comparatively slowly for phases corresponding to absorption. Hence, the initial,uniform phase population develops peaks in regions of phase corresponding to emission and valleys in regions corresponding to absorption. This is to say that the rate of emission is greater than that of absorption, or there results a positive amplification of the radiation intensity.

One can view the interaction of the electron beam with the undulator and radiation fields and the evolution of laser action as proceeding in three overlapping stages. When the bunch enters the undulator and has traversed only a small number of perlods, the predominant effect is a 
redistribution in energy among the electrons, some gaining and some losing energy, thus increasing the energy spread with essentially no net energy transfer to the photon field. Behavior in this stage is first order in the number of periods traversed, as described in Eq. (332). Farther along the undulator, the second stage action occurs; the electrons begin to shift in phase and become spatially modulated. This process depends on $x^{2}$ as indicated by Eq. (337). Finally, more than half the electrons become so iocated in the phase distribution as to transfer energy to the photon field and laser action with positive gain takes place. The actual gain depends on $\eta^{3}$, as shown in $E q$, (329). When the initial electron beam is uniformly distributed in phase and the following linequality is satisfied,

$$
\Omega_{I} \ll \dot{\Phi}_{L_{0}}
$$

the system is sald to be in the "small signal regine". Electrons whose motion satisfies the inequality (321) have open phase space trajectories In the upper half of the diagram Fig. 39. The Stanford experiments fall into the small signal regime, and a calculation of the laser gain in this case begins with the solution of $\mathrm{Eq}$. (319) in the form of a perturbation expansion

$$
\Phi_{L}(t)=\Phi_{L_{0}}+\dot{\Phi}_{L_{0}} t+\frac{\Omega_{L}^{2}}{\dot{\Phi}_{L_{0}} 2} A_{1}(t)+\frac{\Omega_{L}^{4}}{\dot{\Phi}_{L_{0}}^{4}} A_{2}(t)+\cdots \cdot
$$


It is straightforward to show that

$$
A_{1}(t)=\sin \left(\phi_{L_{0}}+\dot{\phi}_{L_{0}} t\right)-t \dot{\Phi}_{L_{0}} \cos \phi_{L_{0}}-\sin \phi_{L_{0}}
$$

and

$$
\begin{aligned}
A_{2}(t)= & \int_{0}^{t} \dot{\Phi}_{L_{0}} d \tilde{t}\left\{\frac{\cos \left(2 \phi_{L_{0}}+2 \dot{\Phi}_{L_{0}} \tilde{t}\right)-\cos 2 \Phi_{L_{0}}}{4}\right. \\
& \left.+\cos \left(\dot{\Phi}_{L_{0}} \bar{t}\right)-1+\dot{\Phi}_{L_{0}} \tilde{t} \cos \Phi_{L_{0}} \sin \left(\Phi_{L_{0}}+\dot{\Phi}_{L_{0}} \bar{t}\right)\right\}
\end{aligned}
$$

The average change in electron energy at time $t$ is given by

$$
\left\langle\frac{\Delta \gamma}{\gamma}\right\rangle=\frac{\lambda_{0}}{4 \pi c} \frac{1}{2 \pi} \int_{0}^{2 \pi} d \phi_{L_{0}}\left[\dot{\phi}_{L}\left(\phi_{L_{0}}, \dot{\phi}_{L_{0}}, t\right)-\dot{\phi}_{L_{0}}\right]
$$

Taking $t=\eta \lambda_{0} / c$ to be the transit time in the undulator and defining

$$
E=\dot{\Phi}_{L_{0}} t=4 \pi \eta\left(\frac{r_{0}-r_{r}}{r_{r}}\right)
$$

from Eqs. (322-325) we obtain

$$
\left\langle\frac{\Delta \gamma}{\gamma}\right\rangle=4 \pi^{3}\left(\frac{\Omega_{L}}{\Omega_{h}}\right)^{4} \eta^{3} f(\xi)
$$

In this equation $\Omega_{h}=2 \pi c / \lambda_{b}[\mathrm{Eq} .(291 \mathrm{a})]$ and the gain function,

$$
\begin{aligned}
f(\xi) & =\frac{1}{\xi^{3}}\left[\cos \xi-1+\frac{1}{2} \xi \sin \xi\right] \\
& =\left[\frac{d}{d x}\left(\frac{\sin ^{2} x}{8 x^{2}}\right)\right]_{x=\xi / 2}
\end{aligned}
$$


is plotted in Fig. 40. Any net change in electron energy (327) arises from the fourth-order term in (322), since the average over the secondorder term vanishes.

Recall from Eq. (297) that in the classical spontaneous emission spectrum for an electron moving through a static undulator field, the lineshape is of form

$$
\frac{\sin ^{2}\left[\pi \eta\left(\frac{\omega}{\omega}-1\right)\right]}{\left(\frac{\omega}{\omega}-1\right)^{2}}
$$

with $\omega_{1 h}=2 \Omega_{h} \gamma_{r}{ }^{2} /\left(1+K_{h}{ }^{2}\right), \omega=2 \Omega_{h} \gamma_{0}{ }^{2} /\left(1+k_{h}{ }^{2}\right)$, and $\left(\gamma_{0}-\gamma_{r}\right) / \gamma_{r} \ll 1$, one sees that $\pi \eta\left(\omega / \omega_{1 h}-1\right)=\xi / 2$, recalling the definitions of $\xi$ and $\Omega_{\mathrm{h}}$ given in Eq. (326) and following Eq. (327). Therefore, the gain function (328) is proportional to the slope of the spontaneous emission lineshape. This conclusion, originally reached by Madey (1971) on the basis of quantum mechanical arguments, was later confirmed by experiment [Elias et al. (1976)], see Fig. 41 .

Let us designate the cross sectional areas of the radiation and electron beams as $\Sigma_{R}$ and $\Sigma_{e}$ respectively. The change in energy of the EM wave is minus $\langle\Delta \gamma / \gamma\rangle$, [Eq. (327)], multiplied by the initial electron energy, $\gamma_{0}$, and by the number of electrons interacting with the EM wave, $\rho_{e} \sum_{R} F_{f} e_{e}$. Here $\rho_{e}$ is the electron density, $\ell_{e}$ the bunch length, and $F_{f}$ the "filling factor",

$$
F_{f}=\left\{\begin{array}{cc}
1 & \text { if } \Sigma_{e}>\Sigma_{R} \\
\Sigma_{e} / \Sigma_{R} & \text { if } \Sigma_{e}<\Sigma_{R}
\end{array}\right.
$$


⿷匚⿱

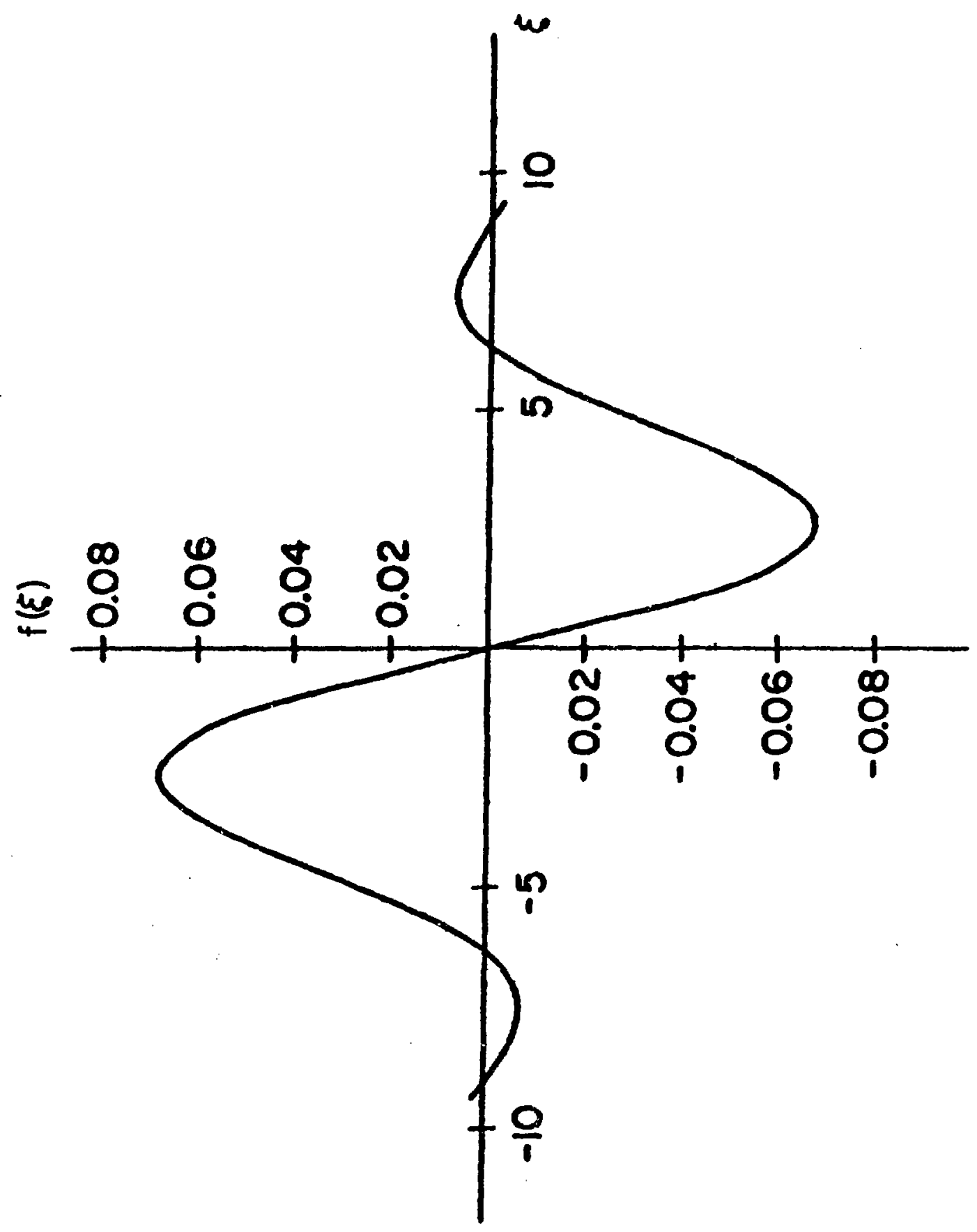




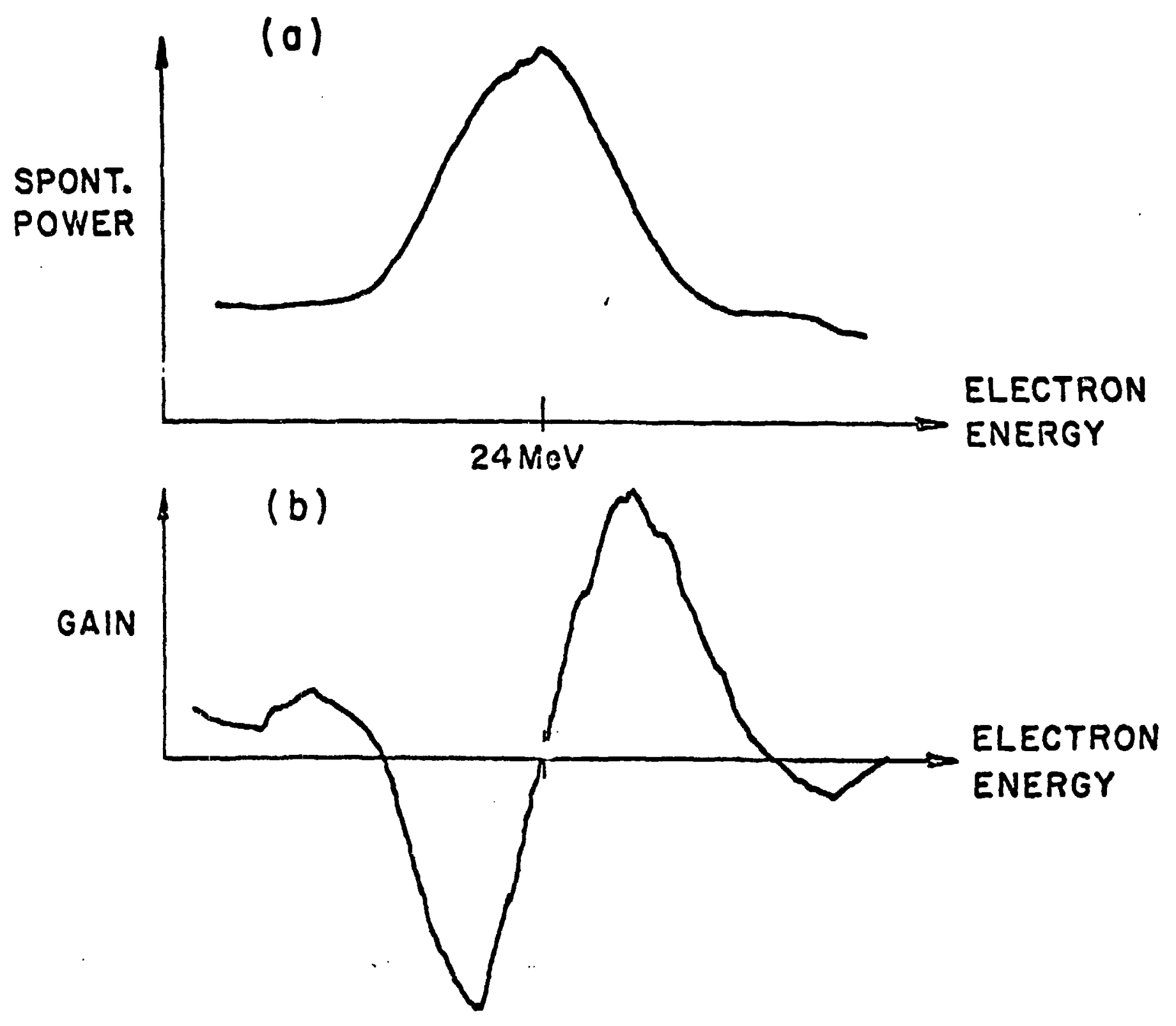


To obtain the gain per pass, G, we divide the above product by the Initial energy in the radiation field, $2 E_{0}^{2} \Sigma_{R} e^{18 \pi}$, obtaining

$$
G=-16 \pi^{2} r_{o} Y_{r}{ }^{-3} \lambda_{o}{ }^{2} k_{h}^{2}\left(F_{f}^{\rho} e\right) \eta^{3} f(\xi)
$$

where $r_{0}-e^{2} / \mathrm{mc}^{2}=2.8 \times 10^{-13} \mathrm{~cm}$ is the classical radius of the electron. If Eq. (312) ts ubed to express the resonant energy $\gamma_{r}$ in terms of the radiation wavelength $\lambda$, we can rewrite (329)

$$
G=-32 \sqrt{2} \pi^{2} r_{0} \lambda_{0}^{1 / 2} \lambda^{3 / 2} \frac{k_{h}^{2}}{\left(1+k_{h}^{2}\right)^{3 / 2}}\left(F_{f} \rho_{e}\right) \eta^{3} f(\xi)
$$

It Is clearly seen that the optical gain is reduced as the radiation wavelength Is decreased.

Elias et al. (1976) measured the optical gain of an FEL (Fig. 38) having the characteristics,

$$
\begin{aligned}
& B_{0}=2.4 \mathrm{kG}, \quad \lambda_{0}=3.2 \mathrm{~cm}, \quad \mathrm{~K}_{h}=0.77 \\
& \eta=160, \quad \lambda=10.6 \mu \mathrm{m}\left(E=24 \mathrm{Me}^{\top /}\right),
\end{aligned}
$$

and Colson (1978) quotes

$$
F_{f e}^{p}=6.4 \times 10^{7} \mathrm{~cm}^{-3}
$$

At maximura gain $\xi_{\max }=2.60$, i.e. $\left(\gamma_{0, \max }-\gamma_{r}\right) / \gamma_{r} \approx 0.2 / \eta$, and $f\left(\xi_{\max }\right)=-0.0675$. The calculated value $G_{\max }=4 \%$ obtained from (330) Is somewhat less than the experimentally observed gain of $7 \%$. 
Let us note that the gain is of ten expressed in terms of the pesk current in amperes, $\Omega_{p}$, of the electron bunch,

$$
r_{0} \rho_{e}=\frac{l_{p}}{l_{A} \Sigma_{e}}
$$

where $\vartheta_{A}=\mathrm{ec} / \mathrm{r}_{\mathrm{o}}=17,000 \mathrm{Amp}$.

Although the laser gain results from the fourth-order term in Eq. (322), the energy spread induced in the electron beam is due predominantly to the second-order $\dot{\phi}_{\text {Lo }}$ term $(\Delta \gamma / \gamma)_{2}$. At the exit of the FEL,

$$
\left(\frac{\Delta \gamma}{\gamma}\right)_{2}=\pi\left(\frac{\Omega_{L}}{\Omega_{h}}\right)^{2} n\left[\frac{\cos \left(\phi_{L_{0}}+\xi\right)-\cos \Phi_{L_{0}}}{\xi}\right]
$$

That the second-order terms make no contribution to the average in Eq. (325) follows from $\Delta \gamma_{2}\left(\Phi_{\mathrm{LO}}+\pi\right)=-\Delta \gamma_{2}\left(\phi_{\mathrm{Lo}}\right)$. Maximizing (332) over Q Lo $_{0}$, we find that

$$
\left(\frac{\Delta y}{y}\right)_{2, \max }= \pm \pi\left(\frac{\Omega_{\mathrm{L}}}{\Omega_{\mathrm{h}}}\right)^{2} \eta\left[\frac{\sin (\xi / 2)}{\xi / 2}\right]
$$

and at $\xi=\xi_{\max },(\Delta \gamma / \gamma)_{2, \max } \cong 2.3\left(\Omega_{L} / \Omega_{h}\right)^{2} \eta$.

For an electron beam which has some spread in energy the average energy change is obtained by folding (327) with the electron energy distribution function. If this distribution attains a width of the order of $(2)^{-1}$, the average energy change is nearly zero. Also, when the maximum energy change for a single electron, as given by (333), becomes of the order of $(2 \eta)^{-1}$, the expression (327) for the average energy gain no longer holds. The conditions $(\Delta \gamma / \gamma)_{\max } \approx(2 \eta)^{-1}$ gives a 
maximum or saturation value for $\Omega_{L} / \Omega_{h}$ on the order of $(2 m)^{-1}$, and with (318) one may determine the saturation value for the radiation fleld atrength $\mathrm{E}_{0}$. As we have seen, the FEI not only changes the electron energy but also modulates the spatial distribution of the electron beam on the scale of the radiation wavelength. The spatial distribution 18 directy related to the phases $\Phi_{I}$ in the bean by

$$
\frac{2 \pi}{\lambda}\left[\zeta(t)-B_{h}{ }^{*} c t\right]=\phi_{L}(t)-\phi_{L 0} t-\phi_{0}-\pi,
$$

a relation which follows from (310) and (311). Introducing the phase distribution function

$$
g\left(\phi_{L}\right)=\frac{1}{2 \pi} \int_{0}^{2 \pi} d \Phi_{L_{0}} \delta\left(\phi_{L}(t)-\phi_{L}\right)
$$

and using Eq. (322),

$$
\Phi_{L}(t) \simeq \Phi_{L o}+\Phi_{L o} t+\left(\frac{\Omega_{L}}{\dot{\Phi}_{L o}}\right)_{A_{1}}^{2}(t)
$$

one determines the phase distribution at the FEL exit, where $t=\eta_{0} / c$, to be

$$
g\left(\Phi_{L}\right) \approx \frac{1}{2 \pi}\left\{1-4 \pi^{2}\left(\frac{\Omega_{L}}{\Omega_{h}}\right]^{2} n\left[\frac{\cos \Phi_{L}+\xi \sin \left(\Phi_{L}-\xi\right)-\cos \left(\phi_{L}-\xi\right)}{\xi^{2}}\right]\right\}
$$

From the definition of $\Phi_{L}$ In Eqs. (309) and (310), we see that in the phase regions $0<s_{L}<\pi(\bmod 2 \pi)$ eleccrons do net work on the radiation fleld. Inftially, the phase distribution is uniform and half the electrons 
are in this phase region. At the exit, the phase distribution is modulated, and the fraction of electrons in phase to lose energy to the radiation is

$$
\int_{0}^{\pi} g\left(\Phi_{L}\right) d \Phi_{L}=\frac{1}{2}-4 \pi\left(\frac{R_{L}}{\Omega_{h}}\right)^{2} \chi^{2} \frac{d}{d \xi}\left[\frac{\sin \xi}{\xi}\right],
$$

a fraction which is greater than one-half when $0<\varepsilon<4.5$.

As emphasized by Colson (1978), Csonka (1978), and Vinokurov and Skrinsky (1977), the spatial modulation of the electron beam exiting from the FEL is potentially of great interest. Under the proper conditions, such a beam incident upon a second device will emit radiation coherentiy, i.e. with the emitted intensity proportional to the square of the electron flux: In particuiar, Vinolarrov and Skrinsky propose what they call an "Optical klystron", an FEC having Its wiggler in two segments separated by a region with specially designed dispersion. The first FEL produces no net energy transfer between the electron beam and the radiation field, but generates an energy spread. The transit time of electrons in the dispergion region depends on electron energy; hence the variation in energy results in a spatial bunching of the electrons. The dispersion section is so designed that the bunched electrons are in proper phase, relative to the phase of the EM wave, to transfer energy coherently to the radiation field in the second FEL segment.

At this point we close our brief discussion of the gain mechanism of the FEL. No attempt has been made to describe the historical development of the fdeas leading to the stimulated enisstion of radiation by free electrons, and we refer the reader to the papexss of Colson (1978) and 
Pellegrini (1979) for references to the important contributions made by many workers in the field. 
Alferov, D. F., Yu. A. Bashmakov and E. G. Bessonov, 1973, Zh. Tekh. F1z. 43, 2126; Eng. trans1. In Sov. Phys. Tech. Phys. 18, 1336 (1974).

Bathow, G., E. Freytag and R. Haense1, 1966, J. Appl. Phys. 37, 33. Blewett, J. P., 1946, Phys. Rev, 69, 87.

Blewett, J. P. and R. Chasman, 1977, J. App1. Phys. 48, 2692. Bruck, H., 1966, Accelerateurs Circulalres des Particules (Presses Univergitaires de France, Paris); English trans1., Los Alamos . Sc1. Lab. Report No. LA-TR-72-10 Rev. Cod1ing, K., and R. P. Madden, 1965, J. App1. Phys. 36, 380. Colsson, R., 1977, Optics Comm. 22, 135. Colson, W. B., 1977, Phys. Lett. 164, 190. Colson, W. B., 1978, One-Body Analysis of Free Electron Lasers, in: Novel Sources of Coherent Radiation, eds. S. F. Jacobs, M. Sargent III, M. 0. Scully (Addis on Wesley, Reading, Mass.) Courant, E. D. and H. S. Snyder, 1958, Ann. Phys. (N.Y.) $3,1$. Csonka, P. L., 1978, Particle Accelerators 8, 225. Deacon, D. A. G., L. R. Ellas, J. M. J. Madey, G. J. Ramian, H. A. Schwettman and T. I. Suith, 1977, Phys. Rev. Lett. 38, 892. Ederer, D. L., E. B. Salomon, S. C. Ebner and R, P. Madden, 1975, J. Res. Nat1. Bur. Stand. 79A, 761. Elder, R. F., A. M. Gurewitach, R. V., Langmuir and H. C. Pollock, 1947, Phys. Rev. 71, 829. EIder, F. R., R. V. Langmult and H. C. Pollock, 1948, Phys. Rev. 74, 52. 
Ellas, L. R., W. M. Falrbank, J. M. J. Madey, H. A. Schwettman, and T. I. Smith, 1976, Phys. Rev, Lett. 36, 717. Green, G. K., 1976, Spectra and Opt1cs of Synchrotron Radiation, Brookhaven Nat'1. Lab. Report BNL 50522 (National Technical Inf. Service, Springfield, VA, U.S.A.).

Iwanenko, D. and I. Pomeranchuk, 1944, Phys. Rev. 65, 343. Jackson, J. D., 1975, Classical Electrodynamics (John Wiley \& Sons, New York); the 1967 edition is useful for some polnts considered here.

Joos, P., 1960, Phys. Rev, Lett. $\underline{4}, 558$.

KIncald, B. M., 1977, i. Appl. Phys. 48, 2684.

Kose, R., 1977, Report of Work1ng Group on Effects of Wigglers on $\varepsilon$, $n$, etc. In Proceedings Wiggler Workshop, Stanford LInear Accel. Center, eds. H. Winick and T. Rnight, SSRP Report No. 77/05, p. III-7.

Llenard, A., 1898, L'Eclairage Elec. 16, 5.

Madey, J. K. J., 1971, J. App 1. Phys. 42, 1906.

MeMtllan, E. M., 1945, Phys. Rev. 68, 144.

Morse, P. M. and H. Feshbach, 1953, Mathods of Theoretical Physics

(McGraw-Hi11, New York).

Morz, H., 1951, J. Appl. Phys. 22, 527.

Motz, H., W. Thon and R. N. Whitehurst, 1953, J. Appl. Phys. 24, 826. Motz, H. and M. Nakamura, 1959, Ann. Phys. 7, 84.

Motz, H. and M. Nakamura, 1960, The Generation of Submillimeter Waves and Fast Wave Amlification, in: Proc. Symp. on Millimeter Waves Microwave Rej. Inst. Symp. Serles IX (Interscience, N.Y.) P. 155. 
Pellegrini, C., 1979, IEEE Trans. Nucl. Sci. NS-26, 3791.

Phillips, R. M., 1960, I.R.E. Trans, Electron Devices, Z, 231.

Purcell, E. M., 1972, Production of Synchrotron Radiation by Wiggler

Magnets, unpublished, reprinted In Proceedings Wiggler Workshop,

Stanford Linear Accel. Center, eds. H. Winick and T. Kntght, SSRP

Report No. 77/05, 1977, p. IV-18.

Robinson, R. W., 1958, Phys. Rev. 111, 373.

Sands, M., 1970, The Physics of Electron Storage Rings-An

Introduction, Stanford Linear Accelerator Report No. SLAC-121 and

Proc. of Int. Sch. of Phys., E. Fermf Course No. 46, 1971, Physics

with Intersecting Storage Rings, ed. B. Touschek (Academic Press,

New York).

Schiff, L. I., 1946, Rev. Sc1. Instr. 17, 6.

Schott, G. A., 1912, Electromagnetic Radlation (Cambridge Univ. Press,

Cambridge).

Schwinger, J., 1946, Phys. Rev. $\underline{70}, 798$.

Schwinger, J., 1949, Phys, Kev. 75, 1912.

Sokolov, A. A. and I. M. Ternov, 1968, Synchrotron Radiation (Pergamon Press, 0xford).

Steffen, K. G., 1965, High Energy Beam Optics (Interscience

Publishers, New York).

Tamboulian, D. H. and P. L. Hartman, 1956, Phys. Rev. 102, 1423.

Vinokurov, N. A. and A. N. Skrinsky, 1977, Institute of Nuclear Physics, Novosibirsk, USSR, Preprint 77/67.

Wintck, H. and T. Kntght, 1977, eds., Proceedings Wiggler Workshop, Stanford LInear Accel. Center, SSRP Report No. 77/05. 


\section{Figure Captions}

Fig. 1 Function $G_{2}\left(\lambda / h_{c}\right)$ of $\mathrm{B}_{3}$. (9) for the radiated power.

Fig. 2 Function $T_{0}\left(\lambda / \lambda_{c}\right)$ of Eq. (11) for the photon flux Integrated over all vertical angles $\psi$.

Fig. 3 Dependence on the vertical angle $\psi$ of the intensities of the parallel (aolid line) and perpendicular (dashed line) polariastion components of the photon flux. The individual curves, plotted for $\lambda / \lambda_{c}=1 / 3,1,10$, and 100 , are Individually normalized to the intensity in the orbital plane $(\psi-0)$ at the respective $\lambda / \lambda_{c}$ value. Note that the abscissa, $\downarrow$ multiplied by the electron energy $\gamma$, makes these curves universal.

Fig. 4 Function $F\left(\lambda / \lambda_{c} ; \psi=0\right)$ of Eq. (12) determining the differential photon flux in the median plane $\psi=0$.

Fig. 5 Praction of rallation, integrated over vertical angle $\psi$, that is parallel polarized, as a function of $\lambda / \lambda_{c}$.

Ft; $6 a$ The angles defining the photon direction, $\underline{n}$, with respect to the instantaneous directions of the electron velocity (y axis) and the electron acceleration ( $x$ axts), as employed by Jackson (1975). $\psi$ is the angle between $\underline{n}$ and the electron velocity vector and $\phi_{x z}$ is the angle between the projection of $\underline{n}$ on the $x-z$ plane and the direction of electron acceleration 조. 
Fig. $6 \mathrm{~b} \psi$ when $\underline{n}$ lies in the $y-z$ plane, the case for Section 2.3 . Here, unlike the condition of $6 a$, the conventions of Schwinger (1949), of Jackson (1975) and of Green (1976) are the same. The photon polarization directions, $\varepsilon_{\perp}$ and $\varepsilon_{\|}$ are appropriate to Fig. 3 and to Eq. (51).

Fig. 7 The quantity $\sigma_{R}$ as a function photon wavelength $\lambda$ for radiation emitted by electrons at several typical values of electron energy and bending radius. With the approximation that the angular distribution of intensity at a given $\lambda$ is a Gaussian, $\sigma_{R}$ is the characteristic angular width.

Fig. 8 A schematic drawing of Aladdin, the storage ring under construction at the University of Wisconsin; some of the principal components of such a device are indicated.

Fig. 9 Coordinates of a point $P$ near the ideal circular orbit, showing the azimuthal distance $s$, the radial coordinate $x$, and the vertical $z$.

Fig. 10 Illustration of Eq. (65), the approximation $d \ell / d s \simeq(\rho+x) / p$.

Fig. 11. The phase ellipse of Eq. (84) in the $y-y^{\prime}$ plane. The coordinates of the four indicated points are given in Eq. (87) in terms of the Twiss parameters.

FIg. 12 One cell of a FODO structure. The amplitude functions corresponding to the thin lens approximation are sketched, showing $\beta_{x}$ at its maximum in focusing quadrupoles $F$ and at its minimum in defocusing quadrupoles $D$. The opposite is the case for $\beta_{z}$.

Fig. 13 Section of a typlcal tune diagram, illustrating the net. of resonance lines which must be avolded in the choice of an operating point. 
Fig. 14 One superperiod of the NSLS 2.5-GeV X-Ray ring. The gradient In the achromatic quadrupole QF is chosen to Incalize the dispersion function $n$ between the dipole magnets BB. Excitation of the quadrupole triplets, Q1, Q2, Q;, bounding the insertion, is such as to produce small values of $B_{x}$ and $B_{2}$ at the insertion center.

Fig. 15 Sketch of the function $\sin \left(\omega_{r f} t+\pi-\phi_{8}\right)$ appearing in Eq. (103), 1llustrating the synchronons phase angle $\phi$. F1g. 16 When an electron gains energy $\delta \varepsilon_{1}$ at the RF cavity, the increase of the electron momentum, $\delta \varepsilon_{1} / c$, is in the longitudinal direction. The angular deviation of the electron motion from the longitudinal. Airection ts therefore changed frow $x$ to $x^{\prime}+\delta x^{\prime}$; as given in Eqs. (127 and 128) $\delta x^{\prime}$ is opposite in sign to $x^{\prime}$.

Fig. 17 Sketch of the "potential energy" function of Eq. (191). The maximum amplitude of stable oscillation corresponds to $\Phi_{0}=\Phi_{\max }$, as indicated.

Fig. 18 The shaded region in $\varepsilon-\tau$ phase space corresponds to stable synchrotron oscillations. Within this "RF bucket" is sketched a typical elliptical trajectory for a stable oscillation, and outside is shown a typical trajectory corresponding to an unstable condition and ultimate loss of the electron. Note that $n$ an electron inftially having excess energy, outside and above the stable region, eventually becomes deficient in energy. 
Fig. 19 Phase space ellipses calculated for the electron beam at seven locations along the orbit in the NSLS $2.5-\mathrm{GeV}$ storage ring. Top and bottom sections of the figure are for $\sigma_{x}, \sigma_{x}$ and $\sigma_{z}, \sigma_{z}$, spaces respectively; the middle section is for $\sigma_{x}, \sigma_{z}$ real space. Each ellipse has the coordinate system origin at its center. Arrows from the superperiod diagram indicate the orbit locations to which the sets of ellipses pertain and the leftmost set is for the center of the insertion region. Note that areas of the $x, x^{\prime}$ and of the $z, z^{\prime}$ ellipses, the horizontal and vertical emittances, are conserved. Electrons are shown traversing the lattice from left to right; if this direction Is reversed, the ellipses would have abscissas inverted relative to those shown.

Fig. 20 Calculated values of $\sigma_{x}, \sigma_{z}, \sigma_{x}$, and $\sigma_{z}$, along a superperfod of the $800-M e V$ Berlin storage ring BESSY in one proposed operating mode. In the lattice diagram B desfgnates bending magnet regions.

Fig. 21 Vertical phase space ellipses $\left(\sigma_{z}, \sigma_{z}\right)$ for a particular electron source and for the photons radiated by $1 t$. The electron ellipse is taken to be symmetrical about the $z, z$ ' axes.

Fig. 22 Illustration of the vertical effective distribution in $z, z^{\prime}$ ' from a point along a source extended in length on the orbit.

a) Electron source ellipse at origin $s=0$ and at a point ts along the orbit;

b) Electron ellipse at $+s$ and its photon ellipse;

c) Photon ellipse at $+s$ and this same ellipse transformed or projected back as an effective source at $s=0$. 
Fig. 23 Behavior in $x, x^{\prime}$ horfzontal phase space of a source having finite extent in $s$, the position along the electron orbit.

a) Relation between position of a source element at $s \neq 0$ and the angle at $s=0$ made by Its $x^{\prime}=0$ ray; the $x$ intercept of this ray at $s=0$ is also shown.

b) Trajectory of phase space ellipses for elements of the extended source; ellipse centers describe the curve $x^{\prime 2}-2 x / \rho=0$, and for the center of an ellipse at $a$, $x^{\prime}=s / \rho$. Ellipse centers in $x, x^{\prime}$ move away from $x=0$ at $s=0$ due to the geometry of the electron orbit; this Is not the case for the $z, z^{\prime}$ vertical characterist1cs, as shown in Fig. 22.

Fig. 24 At left, horizontal phase space properties of an extended source at $D=0$, the center of the source segment, and on the right, the properties measured at an aperture located at distance $D_{1}$ from the source center. The square root gives spread in $x$, at $D=0$, corresponding to a photon source ellipse of a source element located at $s$ away from the center. The dot-dash Iines, horizontal limits of the aperture at $D_{1}$, are shown at left; projected back to $D=0$ they set bounds to the effective source as shown at left.

Fig. 25 On the right, ellipse for vertical phase space $\left(z, z^{\prime}\right)$ characteristics at a slit located at $D=D_{1}$ which views a source at $D=0$ having characteristics given by the ellipse on the left. The slit has vertical opening $2 d$ and is long horizontally. 
Fig. 26 The $z, z^{\prime}$ ellipse for a source at $D=0$ and the ellipse resulting after vertical dispersion as by a two crystal monochromatoz. The crystals are in effect a narrow angular slit, because of their amall angular acceptance $\Delta z^{\prime}$.

Fig. 27 Comparisons between measurement and theory of the angular dependence of the photon flux, as obtained by Codling and Ifadden (1965). The Individual curves are for the parallel and perpendicular polarization components.

Fig. 28 Comparison between measurement and theory of the synchrotrons radiation flux for several electron energies and for wavelengths shorter than $\lambda_{c}$, as obtained by Bathow et al. [1966].

Fig. 29 A several pole, vertical field wiggler installed at the Stanford Synchrotron Radiation Laboratory. The upper magnet poles have been removed; the electron beam vacuum chamber and the lower part of the magnet pole array are shown.

Fig. 30 The magnetic fleld and electron orblt displacement characteristic of a single period transverse wiggler, such as a high field wavelength shifter.

Fig. 31 Electron trajectory in the moving frame $\tilde{F}$, for a transverse undulator when $\mathrm{K}$ is large.

Fig. 32 The function $F_{k}(K)$ [Eqs. $(275,276,281)$ ] for the first, third and fifth harmonics $(k=1,3$, and 5$)$. The wavelengths correspond to $\lambda_{k}=\left(\lambda_{0} / 2 \mathrm{kr}^{2}\right)\left(1+\mathrm{k}^{2} / 2\right)$, for $\lambda_{0}=5 \mathrm{~cm}$ and $\gamma=1370$. For $\gamma ?=50$, the brightness in photons/sec, $1 \%$ bandwidth, $(0.1 \mathrm{mrad})^{2}$, Amp equals $2.1 \times 10^{16} \mathrm{~F}_{k}(\mathrm{~K})$. 
Fig. 33 Spectrum of radiation from a transverse undulator in a forward cone of half-angle $\bar{\theta}_{0}=0.1 / \gamma$ and $\bar{\theta}_{0}=0.4 / \gamma$; the wavelengths are in the region of the first harmonic [Eq. (284)] and the undulator characteristics are $\lambda_{0}=5 \mathrm{~cm}, \gamma=1370, \lambda \mathrm{C}=50$ and $K=1$.

Fig. 34 The integrated [Eq. (285)] spectrum for $K=0.4,1$, and 2 in units of photons/sec, 12 bandwidth, Amp produced by a transverse undulator $\left(\lambda_{0}=5 \mathrm{~cm}, \gamma=1370\right.$, and $\left.\chi=50\right)$.

F1g. 35 Angular distributions of photon intensity at varlous harmonics of radiation produced by a transverse undulator having the same characteristics as that of Fig. 33. Intensities of even harmonics vanish in the forward direction $\left(\theta_{0}=0\right)$.

Fig. 36 Fraction of total energy radiated by a transverse undulator which is found in a cone of half-angle $\bar{\theta}_{0}$ for $K=0.4,1$, and 2 [Eq. (283)]. The undulator paramete:s are $\lambda_{0}=5 \mathrm{~cm}, \gamma=1370$, $x=50$.

Fig. 37 The Stanford free electron laser.

Fig. 38 Experimental arrangement for measuring the amplification by an electron beam in an undulator of Infrared radiation from a carbon-dioxdde laser [Elias et al. (1976)].

Fig. 39 Electron phase space trajectories corresponding to the firstintegral of the mocion [Eq. (319)] in the undulator of a free electron laser.

Fig. 40 Function $f(\xi)$, defined in Eq. (328), which determines the free electron laser gain via Eqs, [(327), (322), (330)]. The normalized energy deviation $\varepsilon$ is defined in Eq. (326). 
Fig. 41 The lower curve shory the gain in a free electron laser, as measured by Elias et al. (1976); this is proportional to the slope of the spontaneous emission lineshape given in the upper curve. 